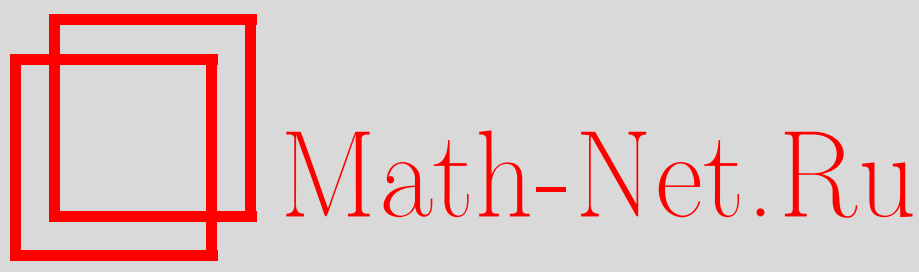

С. К. Багдасаров, Экстремальные функции интегральных функционалов в $H^{\omega}[a, b]$, Изв. РАН. Сер. матем., 1999, том 63, выпуск 3, 3-62

DOI: https://doi.org/10.4213/im241

Использование Общероссийского математического портала Math-Net.Ru подразумевает, что вы прочитали и согласны с пользовательским соглашением http://www . mathnet.ru/rus/agreement

Параметры загрузки:

IP: 54.224 .60 .19

26 апреля 2023 г., $17: 30: 45$ 
УДК 517.988

\author{
С. К. Багдасаров
}

\title{
Экстремальные функции интегральных функционалов в $H^{\omega}[a, b]$
}

В этой статье дается решение дискретного и непрерывного вариантов задачи

$$
\int_{a}^{b} h(t) \psi(t) d t \rightarrow \sup , \quad h \in H^{\omega}[a, b]: h(a)=E_{1}, \quad h(b)=E_{2}
$$

где $H^{\omega}[a, b]$ - класс абсолютно непрерывных функций на $[a, b]$ с общим мажорирующим модулем непрерывности $\omega$. Также обсуждаются приложения полученных результатов в математической экономике (транспортная задача Канторовича-Монжа), теории приближений и численном дифференцировании (чебышевские $\omega$-полиномы и сплайны), конструктивной теории функций (неравенства для $\omega$-перестановок), теории графов (графы перестановок) и теории оптимального управления (теория тотального контроля и задача Фельдбаума-Бушоу).

Библиография: 62 наименования.

\section{§ 0. Введение}

0.1. Убывающие перестановки. Концепщия и свойства убывающих перестановок векторов и неотрицательных функций были впервые систематически изложены в 1934 г. в книге [46].

ОПРЕДЕЛЕНИЕ 0.1. (А) Перестановка $V^{*}=\left(v_{\pi(0)}, v_{\pi(1)}, \ldots, v_{\pi(n)}\right)$ конечномерного вектора $V=\left(v_{0}, v_{1}, \ldots, v_{n}\right)$ называется убывающей перестановкой, если биективное отображение $\pi:(0,1, \ldots, n) \rightarrow(0,1, \ldots, n)$ таково, что $v_{\pi(0)} \geqslant$ $v_{\pi(1)} \geqslant \cdots \geqslant v_{\pi(n)}$.

(В) Функиия распределения $\mu_{f} \quad \mu$-измеримой функции $f: \mathbb{R} \rightarrow \mathbb{R}$ определяется на $\mathbb{R}_{+}$посредством $\mu_{f}(\lambda):=\mu\left\{x \in \mathbb{R}|| f(x) \mid>\lambda, \lambda \in \mathbb{R}_{+}\right\}$, а убивающая перестановка $f^{*}$ функции $f$ дается на $\mathbb{R}_{+}$формулой

$$
f^{*}(t):=\inf \left\{\lambda: \mu_{f}(\lambda) \leqslant t\right\}, \quad t \in \mathbb{R}_{+} .
$$

Другими словами, непрерьвная справа перестановка $f^{*}$ является функцией распределения $m_{\mu_{f}}$ для $\mu_{f}$ относительной меры Лебега $m$ на $\mathbb{R}_{+}$.

Детальное исследование свойств и разнообразных приложений аппарата перестановок было проведено А. Зигмундом [66], Г. Г. Лоренцом [54] и С. Г. Крейном, Ю. И. Петуниным, Е. М. Семеновым [23]; см. также ссылки в книге [38].

(C) С.К. БАГДАСАРОВ 1999 
Н. П. Корнейчук [18] обнаружил приложение аппарата убываюших перестановок к решению задачи максимизации интегральных функционалов в классе $H^{\omega}(\mathbb{I})$ функций на II с модулем непрерывности, мажорируемым фиксированным выпуклым модулем непрерывности $\omega$.

\section{2. Классы $\mathbb{H}^{\omega}[n]$ и $H^{\omega}(\mathbb{I})$.}

ОПРЕДЕЛЕНИЕ 0.2 . Функция $\omega: \mathbb{R}_{+} \rightarrow \mathbb{R}_{+}$называется модулем непрерывности, если:

a) $\omega(0)=0$

b) $\omega(x) \leqslant \omega(y), \quad x<y$;

c) $\omega(x+y) \leqslant \omega(x)+\omega(y), \quad x, y>0$.

Для данного модуля непрерьвности $\omega$ мы определим множества $\mathbb{H}^{\omega}[n]$ векторов и классы $H^{\omega}(\mathbb{I})$ функций, впервые введенные С. М. Никольским [59].

ОПРЕДЕЛЕНИЕ 0.3. Для $p, n \in \mathbb{Z}_{+}, 0 \leqslant p \leqslant n$, положим

$$
\begin{aligned}
& \mathbb{H}^{\omega}[n]:=\left\{\left(z_{0}, z_{1}, \ldots, z_{n}\right) \in \mathbb{R}^{n+1}|| z_{j}-z_{i} \mid \leqslant \omega(j-i), 0 \leqslant i<j \leqslant n\right\}, \\
& \mathbb{H}_{p}^{\omega}[n]:=\left\{\left(z_{0}, z_{1}, \ldots, z_{n}\right) \in \mathbb{H}^{\omega}[n] \mid z_{p}=0\right\} .
\end{aligned}
$$

ОПРЕДЕЛЕНИЕ 0.4. Пусть $r \in \mathbb{Z}_{+}$. Классы функиий $W^{r} H^{\omega}[a, b], \quad H^{\omega}[a, b]$ и $H_{\tau}^{\omega}[a, b]$ для $\tau \in[a, b]$ определяются следуюшим образом:

$$
\begin{aligned}
W^{r} H^{\omega}[a, b] & :=\left\{x \in \mathbb{C}^{r}[a, b] \mid \omega\left(x^{(r)} ; t\right) \leqslant \omega(t), t \in[0, b-a]\right\} \\
H^{\omega}[a, b] & :=W^{0} H^{\omega}[a, b] \\
H_{\tau}^{\omega}[a, b] & :=\left\{x \in H^{\omega}[a, b] \mid x(\tau)=0\right\} .
\end{aligned}
$$

Стандартным примером выпукльх модулей непрерывности $\omega$ и классов $H^{\omega}[a, b]$ служат модули непрерывности Гёльдера $\omega_{\alpha}(t)=t^{\alpha}, \quad 0<\alpha \leqslant 1$, и гельдеровские классы $W^{r} H^{\alpha}[a, b]:=W^{r} H^{\omega_{\alpha}}[a, b]$. Детальный обзор основных результатов в теории периодических классов $W^{r} H^{\omega}(\mathbb{I})$ предложен читателю в обзорной статье [22].

Н.П. Корнейчук также описал экстремальные функции задачи

$$
\int_{a}^{b} h(t) \Psi^{\prime}(t) d t \rightarrow \sup , \quad h \in H_{\tau}^{\omega}[a, b], \quad \tau \in[a, b],
$$

для ядер $\Psi^{\prime} \in \mathbb{L}[a, b]$ с одной переменой знака и нулевым средним на $[a, b]$ и выразил числовое решение задачи $(0.2)$ в терминах перестановок ядра $\Psi(x)$ :

$$
\sup _{h \in H^{\omega}[a, b]} \int_{a}^{b} h(t) \Psi^{\prime}(t) d t=\int_{0}^{b-a} \Psi^{*}(t) \omega^{\prime}(t) d t .
$$

Опираясь на это свойство перестановок, автор расширил понятие перестановок на класс таких абсолютно непрерывных функций $\Psi$ с конечным числом локальных экстремумов, что $\Psi(a)=\Psi(b)=0$ (см. [2]). 
0.3. Содержание и структура статьи. Главной целью работы являются описание строения экстремалей задачи (0.2) для произвольных ядер $\psi=$ $\Psi^{\prime} \in \mathbb{L}[a, b]$ с конечным числом перемен знака, введение понятия перестановки $\Re_{\omega}(\Psi ; \cdot)$ как количественной характеристики задачи $(0.2)$ и демонстрация разнообразных приложений аппарата $\omega$-перестановок в теории приближений, математической экономике, функциональном анализе, теории графов, конструктивной теории функций и теории оптимального управления.

Первым шагом в осушествлении этого плана являются элементарное решение дискретного аналога

$$
\langle V, Z\rangle \rightarrow \sup , \quad Z \in \mathbb{H}_{p}^{\omega}[n], \quad 0 \leqslant p \leqslant n,
$$

задачи (0.2) и соответствующее обобщение конщепции пререстановок на случай произвольных конечномерных векторов. $§ 1$ включает в себя основные результаты работы о структуре решений задачи (0.4) и об экстремальном разложении вектора $V$ на сумму простых векторов с компонентами, меняюшими знак один раз. В $\S 2$ мы обсуждаем строение экстремалей в (0.4) в элементарном случае простых векторов $V$. В $\S 3$ вниманию читателя предлагаются доказательства всех теорем, сформулированных в $\S 1$. Специальные модификации задачи (0.4), широко используемые в разных областях математики, приведены в $\S 4$. В $\S 5$ понятие графа перестановок, тесно связанного с решением задачи (0.4), дает нам возможность получить удобную интерпретацию всех основных результатов $\S 2-4$ в терминах теории графов. Как объяснено в $\S 6$, в случае нулевой суммы компонент вектора $V$ проблема (0.4) оказывается двойственной знаменитой транспортной задаче Канторовича-Монжа из теории линейного программирования. Непрерывная версия (0.2) задачи (0.4) получает свое полное разрешение в $\S 7$. Одно приложение наших результатов в теории аппроксимации в $\S 8$, построение экстремальных функций для задачи Колмогорова-Ландау в классах $W^{r} H^{\omega}(\mathbb{I})$, обобшает конщепщию классических чебьшевских многочленов в $W^{r} H^{\omega}(\mathbb{I})$ на случай нелинейных $\omega$.

Последние два параграфа, $\S 9$ и $\S 10$, образуют добавление к работе. В $\S 9$, базируюшемся на теоремах статьи автора [4], обсуждаются основные неравенства между $\Psi^{*}, \Re_{\omega}(\Psi ; \cdot)$ и перестановками $\Sigma(\Psi ; \cdot)$ Корнейчука абсолютно непрерьвных ядер $\Psi$. Опираясь на результаты работы [6], в завершающем параграфе мы ставим общую задачу теории тотального контроля и описываем приложения результатов настояшей работы к решению задачи Фельдбаума-Бушоу о быстродействиях.

В заключение автор выражает глубокую благодарность Б.С. Митягину и В. М. Тихомирову за их внимание к работе и многочисленные рекомендации, направленные на улучшение статьи; Н. П. Корнейчуку, чьи замечания к [2] использовались при написании данной работы; Р. Дж. Маккену за продуктивные обсуждения связи некоторых аспектов работы с решением транспортной задачи; В. Л. Левину за предоставленные материалы о двойственности задачи о максимизации интегральных функционалов и проблеме Монжа-Канторовича; В. Г. Болтянскому за интересную дискуссию о задачах быстродействия в классической и нестандартной форме. 


\section{§ 1. Перестановки и экстремальные векторы}

В этом параграфе мы опишем решения задачи

$$
\begin{aligned}
& \langle V, Z\rangle \rightarrow \sup , \quad Z \in \mathbb{H}_{0}^{\omega}[n], \\
& \langle V, Z\rangle \rightarrow \sup , \quad Z \in \mathbb{H}_{p}^{\omega}[n], \quad 0 \leqslant p \leqslant n,
\end{aligned}
$$

и введем понятие перестановки для произвольных $n$-мерных векторов. В случае выпуклого модуля непрерывности ссылки $(*)$ и $(* *)$ используются для обозначения задач повсюду в работе. Кроме того, для удобства мы будем придерживаться следуюших обозначений.

ОБОЗНАЧЕНИЕ 1.1. Для $n \in \mathbb{N}$ и фиксированного модуля непрерывности $\omega$ положим

$$
\begin{aligned}
\mathbb{Z}[n]:=\{0,1, \ldots, n\}, \quad \Omega_{n}:=(\omega(0), \omega(1), \ldots, \omega(n)), & \\
S_{n}(V) & :=\sup _{Z \in \mathbb{H}_{0}^{\omega}[n]}\langle V, Z\rangle ; \quad a_{n}:=(\underbrace{a, a, \ldots, a}_{n \text { раз }}), \quad a \in \mathbb{R}, \quad n \in \mathbb{N} .
\end{aligned}
$$

\section{1. Структура экстремалей задачи (**).}

ТЕОРЕМА 1.2. Пусть $\omega-$ выпукльй модуль непрерывности и

$$
V=\left(v_{0}, \ldots, v_{n}\right) \in \mathbb{R}^{n+1} ; \quad \alpha_{i}:=\operatorname{sign} v_{i}, \quad i \in \mathbb{Z}[n] ; \quad S:=\operatorname{sign} \sum_{i=0}^{n} v_{i}
$$

I. Существуют такие неотрицательные числа $v_{i, j}, \quad(i, j) \in \mathbb{Z}[n] \times \mathbb{Z}[n]$, $u \hat{v}_{i}, \quad i \in \mathbb{Z}[n]$, чmo:

i) $\left|v_{i}\right|=\sum_{j=0}^{n} v_{i, j}+\hat{v}_{i}, \quad \sum_{i=0}^{n} \hat{v}_{i}=\sum_{i=0}^{n} v_{i}$

ii) $\alpha_{i} \cdot v_{i, j}+\alpha_{j} \cdot v_{j, i}=0, \quad(i, j) \in \mathbb{Z}[n] \times \mathbb{Z}[n]$;

iii) $\begin{cases}\alpha_{i} \cdot S>0 \Longrightarrow \alpha_{i} \cdot \hat{v}_{i} \geqslant 0, & i \in \mathbb{Z}[n], \\ \alpha_{i} \cdot S \leqslant 0 \Longrightarrow \hat{v}_{i}=0, & i \in \mathbb{Z}[n] .\end{cases}$

II. Если $Z^{*}=\left(z_{0}^{*}, \ldots, z_{n}^{*}\right)$ әкстремален в задаче $(* *)$, то

$$
\begin{array}{rlrl}
v_{i, j} \neq 0 & \Longrightarrow z_{j}^{*}-z_{i}^{*}=\alpha_{j} \cdot \omega(|j-i|), & & (i, j) \in \mathbb{Z}[n] \times \mathbb{Z}[n], \\
\hat{v}_{i} \neq 0 \Longrightarrow z_{i}^{*}=\alpha_{i} \cdot \omega(|i-p|), & & i \in \mathbb{Z}[n] .
\end{array}
$$

III. Пусть $\widehat{V}:=\left(\hat{v}_{0}, \hat{v}_{1}, \ldots, \hat{v}_{n}\right) u V_{i, j}=\left(v_{i, j}^{0}, \ldots, v_{i, j}^{n}\right), \quad 0 \leqslant i<j \leqslant n, \imath \partial e$

$$
v_{i, j}^{l}:= \begin{cases}\alpha_{i} \cdot v_{i, j}, & l=i, \\ \alpha_{j} \cdot v_{j, i}, & l=j, \\ 0, & l \in \mathbb{Z}[n] \backslash\{i, j\} .\end{cases}
$$


Тогда

$$
S_{n}(V)=S_{n}(\widehat{V})+\sum_{0 \leqslant i<j \leqslant n} S_{n}\left(V_{i, j}\right)
$$

IV. Пусть $\hat{v}_{i}:=0, \quad i \in \mathbb{Z} \backslash \mathbb{Z}[n], u \Lambda_{\omega}(V):=\left(\lambda_{0}, \lambda_{1}, \ldots, \lambda_{n}\right) \in \mathbb{R}_{+}^{n+1}$, где $\lambda_{k}=\sum_{i=0}^{n-k} v_{i, i+k}+\hat{v}_{p+k}+\hat{v}_{p-k}, \quad k \in \mathbb{Z}[n]$. Tогдa

$$
S_{n}(V)=\sum_{k=0}^{n} r_{k} \cdot \omega(k)=\left\langle\Lambda_{\omega}(V), \Omega_{n}\right\rangle
$$

\section{2. Экстремальное разложение на сумму простых векторов.}

ОПРЕДЕЛЕНИЕ 1.3. $V=\left(v_{0}, \ldots, v_{n}\right) \in \mathbb{R}^{n+1}$ называется простым вектором, если $\sum_{i=0}^{n} v_{i}=0$ и для некоторого $\varkappa \in\{ \pm 1\}$ сушествуют такие индексы $\left\{i_{j}\right\}_{j=1}^{4}$ : $0 \leqslant i_{1} \leqslant i_{2}<i_{3} \leqslant i_{4} \leqslant n$, что

$$
\operatorname{sign} v_{i}:= \begin{cases}\varkappa, & i_{1} \leqslant i \leqslant i_{2}, \\ -\varkappa, & i_{3} \leqslant i \leqslant i_{4}, \\ 0, & i<i_{1}, \quad i_{2}<i<i_{3}, \quad i>i_{4} .\end{cases}
$$

Положим $\mathbb{I}_{1}(V):=\left(i_{1}, i_{4}\right), \mathbb{I}_{2}(V):=\left[i_{2}, i_{3}\right], \mathbb{I}_{3}(V):=\left[i_{1}, i_{2}\right], \mathbb{I}_{4}:=\left[i_{3}, i_{4}\right]$.

ТЕОРема 1.4. Пусть $\omega-$ выпуклый модуль непрерывности $u \quad V=$ $\left(v_{0}, \ldots, v_{n}\right)$ наделен свойствами

$$
\begin{gathered}
(-1)^{k} v_{i} \geqslant 0, \quad j_{k} \leqslant i<j_{k+1}, \quad k=0, \ldots, l, \\
0=j_{0}<j_{1}<\cdots<j_{l+1}=n+1,
\end{gathered}
$$

$u \sum_{i=0}^{n} v_{i}=0$. Тогда существует разложение $V=\sum_{i=1}^{m} V_{i}$ вектора $V$ на сумму $m \leqslant\lfloor(3 l-1) / 2\rfloor$ простых векторов $\left\{V_{i}\right\}_{i=1}^{m}$ со свойствами:

i) $\sup _{H \in \mathbb{H}_{p}^{\omega}[n]}\langle V, H\rangle=\sum_{i=1}^{m} \sup _{H \in \mathbb{H}_{p}^{\omega}[n]}\left\langle V_{i}, H\right\rangle$;

ii) $\mathbb{I}_{3}\left(V_{l}\right) \in\left[j_{r}, j_{r+1}\right], \quad \mathbb{I}_{4}\left(V_{l}\right) \in\left[j_{q}, j_{q+1}\right], \quad l=1, \ldots, m$, для некоторых $0 \leqslant r, q \leqslant l, \quad r-q$ четно

iii) имеет место одно из следующих включений при $(i, j), \quad 1 \leqslant i<j \leqslant m$ : $\mathbb{I}_{1}\left(V_{i}\right) \subset \mathbb{I}_{2}\left(V_{j}\right)$, или $\mathbb{I}_{2}\left(V_{i}\right) \subset \mathbb{I}_{1}\left(V_{j}\right)$, или $\mathbb{I}_{1}\left(V_{i}\right) \bigcap \mathbb{I}_{1}\left(V_{j}\right)=\varnothing$.

ОПРЕДЕЛЕнИЕ 1.5 . Пусть биекция $V=\left(v_{0}, \ldots, v_{n}\right) \mapsto \stackrel{\stackrel{\leftrightarrow}{V}}{=}\left(\stackrel{\diamond}{v}_{0}, \ldots, \stackrel{\diamond}{v}_{n}\right)$ из $\mathbb{R}^{n+1}$ в себя определяется посредством формул $\hat{v}_{i}:=\sum_{k=i}^{n} v_{k}, i=0, \ldots, n$. Тогда $\omega$-перестановка $\Re_{\omega}(\stackrel{\diamond}{V})$ вектора $\stackrel{\diamond}{V}$ вводится формулой $\Re_{\omega}(\stackrel{\diamond}{V}):=\stackrel{\leftrightarrow}{\Lambda}_{\omega}(V)$ или

$$
\Re_{\omega}(\stackrel{\diamond}{V})=\left(r_{0}, r_{1}, \ldots, r_{n}\right), \quad r_{i}:=\sum_{k=i}^{n} \lambda_{k}, \quad \Lambda_{\omega}(V)=\left(\lambda_{0}, \ldots, \lambda_{n}\right) .
$$


Поскольку отображение $V \mapsto \stackrel{\diamond}{V}$ биективно, $\omega$-перестановка однозначно определена для всех векторов из $\mathbb{R}^{n+1}$. Для выпуклого модуля непрерьвности $\omega$ определим координаты $D(\omega)=\left(d_{0}(\omega), d_{1}(\omega), \ldots, d_{n}(\omega)\right)$ формулами $d_{0}(\omega)=0, d_{i}(\omega)=$ $\omega(i)-\omega(i-1), i=1, \ldots, n$. Преобразование Абеля приводит нас к следующему характеристическому свойству $\omega$-перестановок:

$$
S_{n}(V)=\left\langle\Lambda_{\omega}(V), \Omega_{n}\right\rangle=\left\langle\Re_{\omega}(V), D(\omega)\right\rangle
$$

Соответствуюшие $\omega$-перестановки $\Re_{\omega}(\Psi)$ для абсолютно непрерывных ядер $\Psi$ будут построены в $\S 7$.

\section{§ 2. Решение задачи $(*)$ для простого вектора}

В этом параграфе мы дадим элементарное доказательство теоремы 1.2 для простых векторов $V$. В частности, будет показано, что элементы $\left\{v_{i, j}\right\}_{i, j \in \mathbb{Z}[n]}$ экстремального разложения и $\omega$-перестановки простых векторов не зависят от $\omega$. Зафиксировав $n, m \in \mathbb{N}$, мы будем придерживаться следующих обозначений:

$$
\begin{gathered}
N:=n+m, \quad \mathbb{Q}[n, m]:=\{(i, j) \mid 1 \leqslant i \leqslant n, 1 \leqslant j \leqslant m\}, \\
\mathbb{P}[k]:=\{(i, j) \in \mathbb{Q}[n, m] \mid i+j=N+1-k\}, \quad k=1, \ldots, N-1 .
\end{gathered}
$$

Мы также зафиксируем простой вектор $V=\left(v_{0}, \ldots, v_{n-1}, v_{n}, \ldots, v_{N-1}\right) \in \mathbb{R}_{0}^{N}$ со свойством

$$
v_{i} \geqslant 0, \quad i=0, \ldots, n-1 ; \quad v_{i} \leqslant 0, \quad i=n, \ldots, N-1
$$

2.1. Формулы для элементов разложения. Наборы $\left\{X_{i}\right\}_{i=0}^{n}$ и $\left\{Y_{j}\right\}_{j=0}^{m}$ вводятся посредством

$$
\begin{array}{lll}
X_{0}:=0, & X_{i}:=\sum_{l=0}^{i-1} v_{l}, & i=1, \ldots, n ; \\
Y_{0}:=0, & Y_{j}:=-\sum_{l=1}^{j} v_{N-l}, & j=1, \ldots, m .
\end{array}
$$

Условие $\sum_{i=0}^{N-1} v_{i}=0$ означает, что $X_{n}=Y_{m}$. Для всех $(i, j) \in \mathbb{Q}[n, m]$ положим

$$
\begin{aligned}
X_{i, 0} & :=0, \quad X_{i, j}:=\min \left\{X_{i} ; \max \left\{X_{i-1} ; Y_{j}\right\}\right\} \\
Y_{0, j} & :=0, \quad Y_{j, i}:=\min \left\{Y_{j} ; \max \left\{Y_{j-1} ; X_{i}\right\}\right\} \\
x_{i, j} & :=X_{i, j}-X_{i, j-1}, \quad y_{j, i}:=Y_{j, i}-Y_{j, i-1}
\end{aligned}
$$


Другими словами, $x_{i, j}=y_{j, i}$ - длина интервала $\left[X_{i-1}, X_{i}\right] \cap\left[Y_{j-1}, Y_{j}\right]$ (по определению $|\varnothing|:=0)$. Рис. 2.1 проясняет конструкцию.

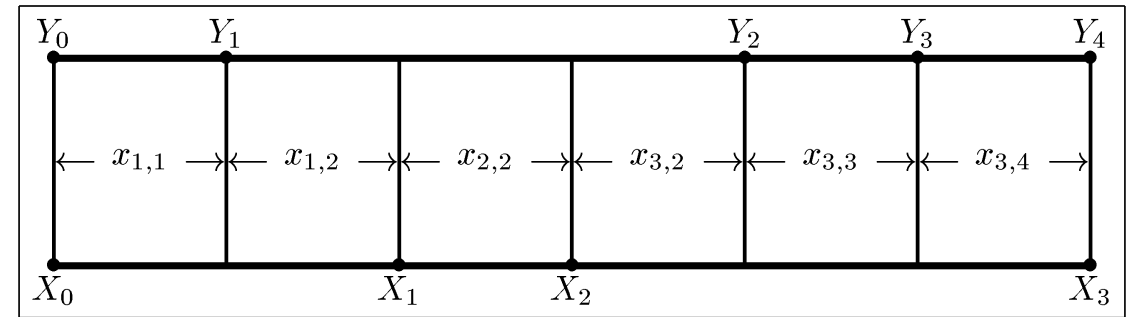

Рис. 2.1. Элементы разложения

Согласно (2.3)-(2.5)

$$
\begin{gathered}
v_{i-1}=\sum_{j=1}^{m} x_{i, j}, \quad 1 \leqslant i \leqslant n ; \quad v_{N-j}=-\sum_{i=1}^{n} y_{j, i}, \quad 1 \leqslant j \leqslant m, \\
x_{i, j}=y_{j, i}, \quad 1 \leqslant i \leqslant n, \quad 1 \leqslant j \leqslant m .
\end{gathered}
$$

2.2. Перестановки простых векторов. Компоненты $\left\{r_{i}\right\}_{i=0}^{N-1}$ nерестанов$\kappa и \Lambda_{\omega}(V)=\left(r_{0}, r_{1}, \ldots, r_{N-1}\right)$ вектора $V$ определяются следуюшим образом: $r_{0}:=$ 0 и

$$
r_{k}:=\sum_{(i, j) \in \mathbb{P}[k]} x_{i, j} \stackrel{(2.7)}{=} \frac{1}{2} \sum_{(i, j) \in \mathbb{P}[k]}\left[x_{i, j}+y_{j, i}\right], \quad k=1, \ldots, N-1,
$$

где $\{\mathbb{P}[k]\}_{k=1}^{N-1}$ были введены в (2.1). Для всех $\mathscr{Z}=\left(z_{0}, \ldots, z_{N-1}\right) \in \mathbb{H}^{\omega}[N-1]$ мы имеем неравенства

$$
\begin{aligned}
\sum_{i=0}^{N-1} v_{i} z_{i} & \stackrel{(2.6)}{=} \sum_{i=1}^{n} \sum_{j=1}^{m}\left(x_{i, j} z_{i-1}-y_{j, i} z_{N-j}\right) \stackrel{(2.7)}{=} \sum_{i=1}^{n} \sum_{j=1}^{m} x_{i, j}\left(z_{i-1}-z_{N-j}\right) \\
& \leqslant \sum_{i=1}^{n} \sum_{j=1}^{m} x_{i, j} \omega(N+1-j-i) \stackrel{(2.8)}{=} \sum_{k=1}^{N-1} r_{k} \omega(k)=\left\langle\Lambda_{\omega}(V), \Omega_{N-1}\right\rangle .
\end{aligned}
$$

Равенства в (2.9) имеют место тогда и только тогда, когда

$$
z_{i-1}-z_{N-j}=\omega(N+1-j-i)
$$

для всех пар $(i, j) \in \mathbb{Q}[n, m]$ с $x_{i, j}>0$. Если $\omega$ - выпукльй модуль непрерывности, то уравнения (2.10) являются необходимымми и достаточными условиями экстремальности вектора $\mathscr{Z}$ в задаче $(*)$.

Ненулевье элементы $\left\{v_{i, j}\right\}_{0 \leqslant i, j \leqslant N-1}$ декомпозиции простого вектора $V \in \mathbb{R}^{N}$ выражаются в терминах $\left\{x_{i, j}, y_{j, i}\right\}_{1 \leqslant i \leqslant n, 1 \leqslant j \leqslant m}$ формулами

$$
v_{i, N-j}=-v_{N-j, i}:=x_{i, j}, \quad(i, j) \in \mathbb{Q}[n, m] .
$$




\section{3. Наблюдения.}

ПРЕДЛОЖЕНИЕ 2.1. Для простого вектора $V=\left(v_{0}, v_{1}, \ldots, v_{N-1}\right) \in \mathbb{R}_{0}^{N}$ nусть $\stackrel{\circ}{v}_{i}:=\sum_{j=0}^{i} v_{j}, \quad i=0, \ldots, N-1$. Задача (*) имеет единственное решение тогда и только тогда, когда

$$
\stackrel{\circ}{v}_{i} \neq \stackrel{\circ}{v}_{j} \quad \forall(i, j): 0 \leqslant i<j \leqslant n .
$$

ДокАЗАТЕЛЬСтво. В следствии 3.3 мы покажем, что если вектор $V$ имеет нулевую компоненту, то задача $(*)$ допускает континуум экстремалей. Если все компоненты $V$ ненулевые, то равенство в (2.12) возможно, только если $\stackrel{\circ}{v}_{i}=\stackrel{\circ}{v}_{j}$ и $\operatorname{sign} v_{i}=-\operatorname{sign} v_{j}$. Пусть $Z^{*}=\left(z_{0}^{*}, \ldots, z_{N-1}^{*}\right)$ - экстремальньй вектор задачи $(*)$, $Z_{1}^{*}=\left(z_{0}^{*}, \ldots, z_{i}^{*}, 0_{j-i}, z_{j+1}^{*}, \ldots, z_{N-1}^{*}\right)$ и

$$
Z_{2}^{*}(\beta)=\left(0_{i+1}, \beta, z_{i+2}^{*}-z_{i+1}^{*}+\beta, \ldots, z_{j}^{*}-z_{i+1}^{*}+\beta, 0_{N-1-j}\right) .
$$

Тогда вектор $\mathscr{Z}_{\beta}:=Z_{1}^{*}+Z_{2}^{*}(\beta)$ экстремален в задаче $(*)$ для всех $\beta \in$ $\left[z_{i}^{*}+\omega(j-i+1)-\omega(j-i), z_{i}^{*}+\omega(j-i)-\omega(j-i-1)\right]$. Это рассуждение доказывает необходимость (2.12). Если выполняются соотношения (2.12), то компоненты экстремального вектора в (*) однозначно определяются уравнениями $(2.10)$.

ЗАмЕчАнИЕ 2.2 . Пусть $k$ - число пар $(i, j)$ в $\mathbb{Q}[n, m]$, для которых $X_{i}=Y_{j}$. Тогда число ненулевых пар $\left(v_{i, j}, v_{j, i}\right)$ в разложении $(2.12)$ равно $N-1-k$.

ЗАмЕчАниЕ 2.3. Если $V$ - простой вектор, то элементы $\left\{v_{i, j}\right\}_{i, j \in \mathbb{N}^{n-1}}$ декомпозиции (2.12) и перестановки $\Lambda_{\omega}(V)$ не зависят от $\omega$.

ЗАмЕчАнИЕ 2.4. Если $V \in \mathbb{Z}^{N}$ - простой вектор с целочисленными компонентами, то элементы $\left\{v_{i, j}\right\}_{i, j \in \mathbb{Z}[N-1]}$ являются неотрицательными цельми числами, т.е. $\Lambda_{\omega}(V) \in \mathbb{Z}_{+}^{N}$.

Теорема 1.2, доказанная в этом параграфе для простых векторов, может рассматриваться как дискретный аналог леммы Корнейчука, приведенной в п. 7.1.

\section{§ 3. Доказательства}

Результаты $\S 2$ служат базисом для индуктивного доказательства теорем 1.2 и 1.4 по числу перемен знака компонент векторов $V$. Доказательство индуктивного шага будет проведено отдельно для векторов с четны.м и нечетным числом перемен знака в компонентах в п. 3.2 и п. 3.3 соответственно. Некоторые свойства экстремальных векторов задачи (*) раскрываются в п. 3.1.

В пп. 3.1-3.3 теоремы 1.2 и 1.4 будут доказаны для векторов $V=\left(v_{0}, \ldots, v_{n}\right)$ с $\sum_{i=0}^{n} v_{i}=0$, а случай $\sum_{i=0}^{n} v_{i} \neq 0$ рассматривается в п. 3.4 .

ЗАМЕЧАНИЕ 3.1. Из неравенства

$$
\left|\left\langle V_{2}, Z\right\rangle-\left\langle V_{1}, Z\right\rangle\right| \leqslant\left\|V_{2}-V_{1}\right\|_{l_{1}^{n+1}} \omega(n), \quad V_{1}, V_{2} \in \mathbb{R}^{n+1}, \quad Z \in \mathbb{H}_{0}^{\omega}[n]
$$


следует, что

$$
\left|S_{n}\left(V_{2}\right)-S_{n}\left(V_{1}\right)\right| \leqslant\left\|V_{2}-V_{1}\right\|_{l_{1}^{n+1} \omega}(n) .
$$

Следовательно, достаточно доказать теоремы 1.2 и 1.4 для векторов $V$ из всюду плотного подмножества в $\mathbb{R}_{0}^{n+1}$, в частности для векторов с ненулевы.ми координатами.

3.1. Свойства экстремальных векторов задачи (*). Мы зафиксируем вектор $V=\left(v_{0}, \ldots, v_{n}\right) \in \mathbb{R}_{0}^{n+1}$ с $v_{i} \neq 0, i \in \mathbb{Z}[n]$. Как обычно, $\alpha_{i}:=\operatorname{sign} v_{i}$, $i \in \mathbb{Z}[n]$.

ЛЕмма 3.2. Если $Z^{*}=\left(z_{0}^{*}, \ldots, z_{n}^{*}\right) \in \mathbb{H}_{0}^{\omega}[n]$ әкстремален в $(*)$, то для любого $i \in \mathbb{Z}[n]$ существует такой индекс $j=j(i) \in \mathbb{Z}[n]$, что

$$
\alpha_{j} \cdot \alpha_{i}=-1, \quad z_{j}^{*}-z_{i}^{*}=\alpha_{j} \omega(|j-i|)
$$

Eсли $\alpha_{l}=\alpha_{m}$ nрu $l, m \in \mathbb{Z}[n]: l \neq m, m o\left|z_{l}^{*}-z_{m}^{*}\right|<\omega(|l-m|)$.

ДокАЗАТЕЛЬСТво. Если при некоторых $j \in \mathbb{Z}[n]$ и $\delta>0$

$$
\alpha_{j}\left(z_{j}^{*}-z_{i}^{*}\right) \leqslant \omega(|j-i|)-\delta, \quad i \in \mathbb{Z}[n] \backslash\{j\},
$$

TO

$$
\langle\widehat{Z}, V\rangle=\left\langle Z^{*}, V\right\rangle+\left|v_{j}\right| \delta=S_{n}(V)+\left|v_{j}\right| \delta>S_{n}(V)
$$

для $\widehat{Z}:=\left(z_{0}^{*}, \ldots, z_{j-1}^{*}, z_{j}^{*}+\alpha_{j} \cdot \delta, z_{j+1}^{*}, \ldots, z_{n}^{*}\right) \in \mathbb{H}_{0}^{\omega}[n]$, что противоречит экстремальности $Z^{*}$ в $(*)$.

Для всех индексов $j \in \mathbb{Z}[n]$ пусть $i, k \in \mathbb{Z}[n]$ таковы, что

$$
z_{j}^{*}-z_{i}^{*}=\alpha_{j} \omega(|j-i|), \quad z_{i}^{*}-z_{k}^{*}=\alpha_{i} \omega(|i-k|) .
$$

Покажем, что $\alpha_{i} \cdot \alpha_{j}=-1$. Действительно, если $\alpha_{j}=\alpha_{i}$, мы приходим к противоречию с включением $Z^{*} \in \mathbb{H}_{0}^{\omega}[n]$ :

$$
\left|z_{j}^{*}-z_{k}^{*}\right|=[\omega(|j-i|)+\omega(|i-k|)]>\omega(|j-k|) .
$$

Наконец, пусть $l, m \in \mathbb{Z}[n], l<m$, таковы, что

$$
\alpha_{l}=\alpha_{m}, \quad z_{l}^{*}-z_{m}^{*}=\tau \cdot \omega(m-l), \quad \tau \in\{ \pm 1\}
$$

Пусть $r \in \mathbb{Z}[n] \backslash\{l, m\}$ - индекс со свойствами: $\alpha_{r}=-\alpha_{l}$ и

i) $z_{l}^{*}-z_{r}^{*}=\alpha_{l} \omega(|l-r|)$ при $\tau=\alpha_{l}$;

ii) $z_{m}^{*}-z_{r}^{*}=\alpha_{m} \omega(|m-r|)$ при $\tau=-\alpha_{m}$.

В первом случае $\left|z_{m}^{*}-z_{r}^{*}\right|=[\omega(m-l)+\omega(|l-r|)]>\omega(|m-r|)$, а во втором $\left|z_{l}^{*}-z_{r}^{*}\right|=[\omega(m-l)+\omega(|m-r|)]>\omega(|l-r|)$. Оба неравенства противоречат включению $Z^{*} \in \mathbb{H}_{0}^{\omega}[n]$.

Далее мы покажем, что в случае векторов $V$ с нулевой координатой задача $(*)$ имеет континуум решений. 
СлеДСТВИЕ 3.3. Пусть задача (*) имеет единственное решение для вектора $V=\left(v_{0}, \ldots, v_{n}\right)$. Тогда ни одна координата $\left\{v_{i}\right\}_{i=0}^{n}$ не равна нулю.

ДокАЗАТЕЛЬСТво. Если $V$ имеет нулевую координату, то для всех $\varepsilon>0$ и $\tau= \pm 1$ мы введем вектор $V^{\tau}(\varepsilon)=\left(v_{0}^{\tau}(\varepsilon), \ldots, v_{n}^{\tau}(\varepsilon)\right)$ :

$$
v_{i}^{\tau}(\varepsilon):=v_{i}, \text { если } v_{i} \neq 0, \quad v_{i}^{\tau}(\varepsilon):=\tau \cdot \varepsilon, \text { если } v_{i}=0 .
$$

Для $\tau= \pm 1$ пусть $Z^{\tau}(\varepsilon) \in \mathbb{H}_{0}^{\omega}[n]$ - вектор со свойствами $\left\langle Z^{\tau}(\varepsilon), V^{\tau}(\varepsilon)\right\rangle=$ $S_{n}\left(V^{\tau}(\varepsilon)\right)$ и $\mathscr{Z}^{\tau}=\lim _{k \rightarrow \infty} Z^{\tau}\left(\varepsilon_{k}\right)$ для некоторой сходяшейся последовательности $\varepsilon_{k} \downarrow 0, k \uparrow \infty$. Пусть $v_{i}=0$ - нулевая координата $V$. Согласно лемме 3.2 $\mathscr{Z}^{\tau}=\left(z_{0}^{\tau}, z_{1}^{\tau}, \ldots, z_{n}^{\tau}\right)$, предельный вектор последовательностей $\left\{Z^{\tau}\left(\varepsilon_{k}\right)\right\}_{k \in \mathbb{N}}$ при $\tau= \pm 1$ наделен следуюшим свойством: сушествуют такие $l_{\tau} \in \mathbb{Z}[n]: l \neq i$, что $z_{i}^{\tau}-z_{l_{\tau}}^{\tau}=\tau \cdot \omega\left(\left|i-l_{\tau}\right|\right)$. Это свойство в сочетании с полуаддитивностью $\omega$ означает, что $\mathscr{Z}^{1} \neq \mathscr{Z}^{-1}$. Действительно, если $\mathscr{Z}^{1}=\mathscr{Z}^{-1}=\mathscr{Z}=\left(z_{0}, \ldots, z_{n}\right)$, то

$\left|z_{l_{1}}-z_{l_{-1}}\right|=\left|\left(z_{l_{1}}-z_{i}\right)+\left(z_{i}-z_{l_{-1}}\right)\right|=\omega\left(\left|i-l_{1}\right|\right)+\omega\left(\left|i-l_{-1}\right|\right)>\omega\left(\left|l_{1}-l_{-1}\right|\right)$,

что противоречит включению $\mathscr{Z} \in \mathbb{H}_{0}^{\omega}[n]$.

ЗАмЕчАНИЕ 3.4. Лемма 3.2 и следствие 3.3 справедливы для всех полуаддитивных модулей непрерывности $\omega$. Начиная с этого момента, в данной работе мы будем рассматривать только строго выпуклые модули непрерывности $\omega$.

В наших рассужлениях мы часто будем использовать следующее наблюдение.

Лемма 3.5. Пусть $Z=\left(z_{0}, \ldots, z_{n}\right) \in \mathbb{H}_{0}^{\omega}[n] u$

$$
\left|z_{i_{2}}-z_{i_{1}}\right|=\omega\left(i_{2}-i_{1}\right), \quad\left|z_{i_{4}}-z_{i_{3}}\right|=\omega\left(i_{4}-i_{3}\right)
$$

для некоторых $\left\{i_{j}\right\}_{j=1}^{4}, \quad i_{1}<i_{2}, i_{3}<i_{4}$. Тогда или $\left(i_{1}, i_{2}\right) \cap\left[i_{3}, i_{4}\right]=\varnothing$, или $\left[i_{1}, i_{2}\right] \subseteq\left[i_{3}, i_{4}\right]$, или $\left[i_{3}, i_{4}\right] \subseteq\left[i_{1}, i_{2}\right]$.

Структурный анализ экстремалей задачи $(*)$ распадается на рассмотрение двух случаев: векторов $V$ с четным и нечетным числом перемен знака. В обеих ситуациях мы используем индуктивный аргумент: предполагая, что теоремы 1.2 и 1.4 уже установлены для векторов $V$ с $k \leqslant r-1$ переменами знака в координатах, мы докажем обе теоремы для всех векторов $V$ с $r$ переменами знака.

3.2. Четное число перемен знака: $\alpha_{0}=\alpha_{n}$. В данном пункте мы фиксируем вектор $V \in \mathbb{R}_{0}^{n+1}$ с ненулевыми координатами и с четным числом $r=2 l$ перемен знака. Будет установлено существование такого индекса $L \in \mathbb{Z}[n]$ и векторов $V_{1} \in$ $\mathbb{R}_{0}^{L+1}$ и $V_{2} \in \mathbb{R}_{0}^{n-L+1}$, что:

i) $V=\left(V_{1}, 0_{n-L}\right)+\left(0_{L}, V_{2}\right)$;

ii) $S_{n}(V)=S_{L}\left(V_{1}\right)+S_{n-L}\left(V_{2}\right)$. 
Tеорема 3.6. Если $\alpha_{0}=\alpha_{n}$, то существуют такие индекс $L \in \mathbb{Z}[n]$ и числа $v^{\prime}, v^{\prime \prime}: v_{L}=v^{\prime}+v^{\prime \prime}$, что $\alpha_{L}=-\alpha_{0} u$

i) $\alpha_{L} \cdot v^{\prime} \geqslant 0, \quad \alpha_{L} \cdot v^{\prime \prime} \geqslant 0$

ii) $\sum_{i=0}^{L-1} v_{i}+v^{\prime}=0=v^{\prime \prime}+\sum_{i=L+1}^{n} v_{i}$;

iii) $S_{n}(V)=S_{L}\left(V_{1}\right)+S_{n-L}\left(V_{2}\right), \quad$ zдe $\left\{\begin{array}{l}V_{1}=\left(v_{0}, \ldots, v_{L-1}, v^{\prime}\right), \\ V_{2}=\left(v^{\prime \prime}, v_{L+1}, \ldots, v_{n}\right) .\end{array}\right.$

ДокАЗАТЕЛЬСТво. Пусть $Z^{*}=\left(z_{0}^{*}, \ldots, z_{n}^{*}\right)$ - экстремальный вектор задачи $(*)$. Лемма 3.2 гарантирует существование максимального индекса $L$ в $\mathbb{Z}[n]$ со свойством $\alpha_{L}=-\alpha_{0}$ и $z_{L}^{*}-z_{0}^{*}=\alpha_{L} \omega(L)$. Тогда следуюшие неравенства для некоторого $\Delta: 0<\Delta<\omega(1)$, вытекают из леммы 3.5 :

$$
\begin{array}{ll}
\left|z_{j}^{*}-z_{i}^{*}\right| \leqslant \omega(j-i) & \forall(i, j): 0 \leqslant i \leqslant L<j \leqslant n ; \\
\alpha_{L}\left(z_{k}^{*}-z_{L}^{*}\right)<\omega(k-L)-\Delta \quad \forall k \in \mathbb{Z}[n] \backslash\{L\} .
\end{array}
$$

Если мы предположим, что $\operatorname{sign} \sum_{i=L}^{n} v_{i}=-\alpha_{L}$, то

$$
\langle\bar{Z}, V\rangle=\Delta \cdot\left|\sum_{i=L}^{n} v_{i}\right|+\left\langle Z^{*}, V\right\rangle>S_{n}(V)
$$

для $\bar{Z}=\left(z_{0}^{*}, \ldots, z_{L-1}^{*}, z_{L}^{*}-\alpha_{L} \Delta, z_{L+1}^{*}-\alpha_{L} \Delta, \ldots, z_{n}^{*}-\alpha_{L} \Delta\right) \in \mathbb{H}_{0}^{\omega}[n]$.

Если мы предположим, что $\operatorname{sign} \sum_{i=L+1}^{n} v_{i}=\alpha_{L}$, то

$$
\langle\widetilde{Z}, V\rangle=\Delta \cdot\left|\sum_{i=L+1}^{n} v_{i}\right|+\left\langle Z^{*}, V\right\rangle>S_{n}(V)
$$

для $\widetilde{Z}=\left(z_{0}^{*}, \ldots, z_{L}^{*}, z_{L+1}^{*}+\alpha_{L} \Delta, z_{L+1}^{*}+\alpha_{L} \Delta, \ldots, z_{n}^{*}+\alpha_{L} \Delta\right) \in \mathbb{H}_{0}^{\omega}[n]$.

Оба противоречия с экстремальностью $Z^{*}$ приводят нас к заключению, что $\alpha_{L} \cdot \sum_{i=L}^{n} v_{i} \geqslant 0$ и $\alpha_{L} \cdot \sum_{i=L+1}^{n} v_{i} \leqslant 0$. Следовательно, существуют $v^{\prime}, v^{\prime \prime} \geqslant 0$ со свойствами $(3.1), \mathrm{i})$, ii). Тогда для $V_{1}, V_{2}: V=V_{1}+V_{2}$, определенных в $(3.1)$, iii), мы имеем

$$
S_{n}(V) \leqslant S_{L}\left(V_{1}\right)+S_{n-L}\left(V_{2}\right) .
$$

Пусть векторы $Z_{1}=\left(z_{0}^{1}, \ldots, z_{L}^{1}\right) \in \mathbb{H}_{0}^{\omega}[L], Z_{2}=\left(z_{0}^{2}, \ldots, z_{n-L}^{2}\right) \in \mathbb{H}_{0}^{\omega}[n-L]$ обладают свойствами

$$
\left\langle Z_{1}, V_{1}\right\rangle=S_{L}\left(V_{1}\right), \quad\left\langle Z_{2}, V_{2}\right\rangle=S_{n-L}\left(V_{2}\right) .
$$

Положим

$$
\mathscr{Z}:=\left(Z_{1}, 0_{n-L}\right)+\left(0_{L}, Z_{2}\right)=\left(z_{0}^{1}, \ldots, z_{L-1}^{1}, z_{L}^{1}, z_{1}^{2}, \ldots, z_{n-L}^{2}\right) .
$$


Из неравенств (3.2) следует, что вектор $Z_{\gamma}:=(1-\gamma) Z^{*}+\gamma \mathscr{Z}$ принадлежит $\mathbb{H}_{0}^{\omega}[n]$ для всех $\gamma \in\left(0,(2 \omega(n))^{-1} \Delta\right)$. Более того,

$$
S_{n}(V) \geqslant\left\langle Z_{\gamma}, V\right\rangle \stackrel{(3.4)}{=}(1-\gamma) S_{n}(V)+\gamma\left(S_{L}\left(V_{1}\right)+S_{n-L}\left(V_{2}\right)\right) \stackrel{(3.3)}{\geqslant} S_{n}(V) .
$$

Следовательно, $S_{n}(V)=S_{L}(V)+S_{n-L}(V)$.

Пусть $n_{1}:=L, n_{2}:=n-L, V_{k}=\left(v_{1}^{k}, \ldots, v_{n_{k}}^{k}\right), k=1,2$. Согласно нашему индуктивному допущению существуют такие разложения для $k=1,2$

$$
\left|v_{i}^{k}\right|=\sum_{j=0}^{n_{k}} v_{i, j}^{k}, \quad v_{i, j}^{k} \geqslant 0, \quad 0 \leqslant i, j \leqslant n_{k},
$$

что:

i) $\alpha_{i} \cdot v_{i, j}^{k}+\alpha_{j} \cdot v_{j, i}^{k}=0, \quad 0 \leqslant i \leqslant j \leqslant n_{k}$;

ii) $v_{i, j}^{k} \neq 0 \Longrightarrow z_{j}^{*}-z_{i}^{*}=\alpha_{j} \omega(|j-i|), \quad 0 \leqslant i, j \leqslant n_{k}$.

Если $V=\left(v_{0}, \ldots, v_{n}\right)$, то по определению $V_{1}, V_{2}$

$$
v_{i}=v_{i}^{1}, \quad 0 \leqslant i \leqslant n_{1}-1 ; \quad v_{n_{1}}=v_{n_{1}}^{1}+v_{0}^{2} ; \quad v_{i}=v_{i-n_{1}}^{2}, \quad n_{1}+1 \leqslant i \leqslant n .
$$

Таким образом, декомпозиция $\left\{v_{i, j}\right\}_{i, j \in \mathbb{Z}[n]}$ вектора $V$ дается формулами

$$
v_{i, j}= \begin{cases}0, & i<L<j, \\ v_{i, j}^{1}, & 0 \leqslant i \leqslant j \leqslant L, \\ v_{i-L, j-L}^{2}, & L \leqslant i \leqslant j \leqslant n,\end{cases}
$$

и $v_{j, i}=-v_{i, j}, j \geqslant i$. Это замечание завершает проверку индуктивного шага в доказательстве теоремы 1.2 для векторов с четным числом перемен знака.

Для $k=1,2$ пусть $\left\{V_{i}^{k}\right\}_{i=1}^{m_{k}}$ - семейства простых векторов, ассоциируемых с задачей $(*)$ при $V=V_{k}$ и наделенных свойствами, описанными в теореме 1.4. Тогда соответствуюшее разложение $\left\{V_{i}\right\}_{i=1}^{m}$ вектора $V$ дается формулами

$$
V=\sum_{k=1}^{2} \sum_{i=1}^{m_{k}} \widehat{V}_{i}^{k}, \quad \begin{cases}\widehat{V}_{i}^{1}:=\left(V_{i}^{1}, 0_{n-L}\right), & 1 \leqslant i \leqslant m_{1} \\ \widehat{V}_{i}^{2}:=\left(0_{L}, V_{i}^{2}\right), & 1 \leqslant i \leqslant m_{2}\end{cases}
$$

Через $l_{1}, l_{2}, l$ обозначим число перемен знака векторов $V_{1}, V_{2}, V$ соответственно. Тогда $l=l_{1}+l_{2}$ и $m_{k} \leqslant\left\lfloor\left(3 l_{k}-1\right) / 2\right\rfloor$ по нашему индуктивному предположению. Следовательно,

$$
m=m_{1}+m_{2} \leqslant \sum_{k=1}^{2}\left\lfloor\frac{3 l_{k}-1}{2}\right\rfloor \leqslant\left\lfloor\frac{\left[3\left(l_{1}+l_{2}\right)-1\right]}{2}\right\rfloor=\left\lfloor\frac{3 l-1}{2}\right\rfloor,
$$


поскольку $l_{1}$ и $l_{2}$ имеют одинаковую четность. Это наблюдение завершает проверку индуктивного шага в доказательстве теоремы 1.4.

Пусть индекс $L \in \mathbb{Z}[n]$, числа $v^{\prime}, v^{\prime \prime}: v^{\prime}+v^{\prime \prime}=v_{L}$, и векторы $V_{1}$ и $V_{2}$ определены в теореме 3.6 (см. рис. 3.1 ).

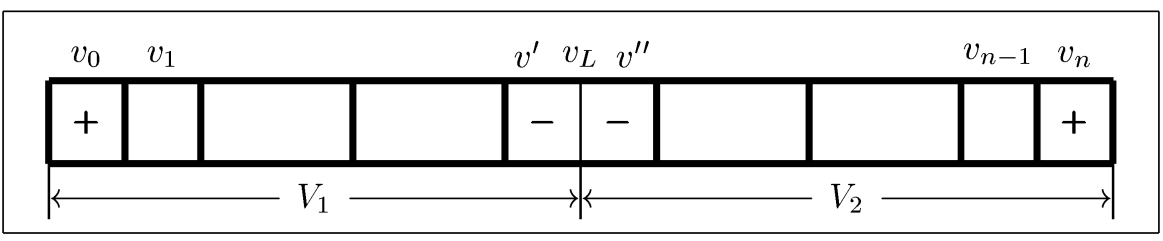

Рис. 3.1. Случай векторов с четным числом перемен знака

СЛЕДСТВИЕ 3.7. Пусть $Z^{*}=\left(z_{0}^{*}, \ldots, z_{n}^{*}\right)$ - әкстремальньй вектор задачи (*) $u$

$$
Z_{1}^{*}:=\left(z_{0}^{*}, z_{1}^{*}, \ldots, z_{L}^{*}\right), \quad Z_{2}^{*}:=\left(0, z_{L+1}^{*}-z_{L}^{*}, \ldots, z_{n}^{*}-z_{L}^{*}\right)
$$

Тогда $Z^{*}$ является единственной экстремалью в (*) тогда и только тогда, когда при $k=1,2$ вектор $Z_{k}^{*}$ - единственное решение задачи

$$
\left\langle Z, V_{k}\right\rangle \rightarrow \sup , \quad Z \in \mathbb{H}_{0}^{\omega}\left[n_{k}\right]
$$

əде $n_{1}=L$ u $n_{2}=n-L$.

ДокаЗАТЕльство. Необходимость. Опираясь на (3.2), мы показали в доказательстве теоремы 3.6, что если векторы $Z_{1}$ и $Z_{2}$ наделены свойствами $(3.4)$ и $\mathscr{Z}$ определен в $(3.5)$, то $Z_{\gamma}:=(1-\gamma) Z^{*}+\gamma \mathscr{Z}$ принадлежит классу $H_{0}^{\omega}$ для всех $\gamma \in[0, \varepsilon]$ и экстремален в задаче $(*)$. Единственность экстремали в $(*)$ означает, что $Z_{k}=Z_{k}^{*}, k=1,2$.

Достаточность. Пусть $\widehat{V}_{1}:=\left(V_{1}, 0_{n-L}\right), \widehat{V}_{2}:=\left(0_{L}, V_{2}\right)$. Тогда $V=V_{1}+V_{2}$ и

$$
\langle V, Z\rangle=\left\langle\widehat{V}_{1}, Z\right\rangle+\left\langle\widehat{V}_{2}, Z\right\rangle \leqslant\left\langle V_{1}, Z^{*}\right\rangle+\left\langle V_{2}, Z^{*}\right\rangle=\left\langle V, Z^{*}\right\rangle
$$

для всех $Z \in \mathbb{H}_{0}^{\omega}[n]$. Ввиду единственности и формы экстремалей задач (3.10) при $k=1,2$ мы имеем равенства в (3.11) тогда и только тогда, когда $Z=Z^{*}$.

ЗАмЕчАниЕ 3.8. Если задача (*) имеет единственное решение, то $v^{\prime} \neq 0$, $v^{\prime \prime} \neq 0$. Действительно, если $v^{\prime}=0$, то $V_{1}$ имеет нулевую координату и согласно следствию 3.3 задача (3.10) при $k=1$ имеет континуум решений. Если $v^{\prime \prime}=0$, то $V_{2}$ имеет нулевую компоненту. 
3.3. Нечетное число перемен знака: $\alpha_{0}=-\alpha_{n}$. В этом пункте $V \in \mathbb{R}_{0}^{n+1}-$ вектор с ненулевыми координатами и нечетным числом $r=2 l+1$ перемен знака. Мы докажем существование таких индексов $K, L, M: 0 \leqslant K<L<M \leqslant n$, векторов $V_{1} \in \mathbb{R}_{0}^{L-K+1}, V_{2} \in \mathbb{R}_{0}^{M-L+1}$ и простого вектора $V_{3} \in \mathbb{R}_{0}^{n+1}$ формы $\left(U^{\prime}, 0_{M-K-1}, U^{\prime \prime}\right)$ для некоторых $U^{\prime} \in \mathbb{R}^{K+1}$ и $U^{\prime \prime} \in \mathbb{R}^{n-M+1}$, что (см. рис. 3.2$)$

$$
\begin{aligned}
V & =\left(0_{K}, V_{1}, 0_{n-L}\right)+\left(0_{L}, V_{2}, 0_{n-M}\right)+V_{3}, \\
S_{n}(V) & =S_{L-K}\left(V_{1}\right)+S_{M-L}\left(V_{2}\right)+S_{n}\left(V_{3}\right) .
\end{aligned}
$$

Обозначим

$$
\begin{gathered}
j_{1}=\max \left\{j \in \mathbb{Z}[n] \mid \alpha_{i}=\alpha_{0}, 0 \leqslant i \leqslant j\right\}, \\
j_{2}=\min \left\{j \in \mathbb{Z}[n] \mid \alpha_{i}=\alpha_{n}, j \leqslant i \leqslant n\right\}, \\
\mathscr{M}:=\left\{(i, j) \in \mathbb{Z}[n] \times \mathbb{Z}[n] \mid z_{j}^{*}-z_{i}^{*}=\alpha_{j} \omega(j-i), 1 \leqslant i \leqslant j_{1}, j_{2} \leqslant j \leqslant n\right\} .
\end{gathered}
$$

Возникают две возможности.

1) $\mathscr{M}=\varnothing$. Придерживаясь обозначений п. 3.2 , определим индекс $L$ и построим векторы $V_{1}$ и $V_{2}$, как в п. 3.2. Если $\mathscr{M}=\varnothing$, то векторы $V_{1}$ и $V_{2}$ имеют по меньшей мере одну перемену знака, и мы можем применить индукцию, как и в п. 3.2. В этом случае $V_{3}=0_{n+1}, K:=0$ и $M:=n$.

2) $\mathscr{M} \neq \varnothing$. Из леммы 3.5 следует, что

$$
\mathscr{M}=\left\{\left\{\left(k_{i}, m_{i}\right)\right\}_{i=1}^{r} \mid j_{2} \leqslant m_{1} \leqslant \cdots \leqslant m_{r} \leqslant n, j_{1} \geqslant k_{1} \geqslant \cdots \geqslant k_{r} \geqslant 1\right\} .
$$

Проверка истинности следуюшего результата проводится аналогично доказательству теоремы 3.6 .

Teоpema 3.9. Пусть $\alpha_{0}=-\alpha_{n}, \mathscr{M} \neq \varnothing u K:=k_{1}, M:=m_{1}$. Существуют mакие $u^{\prime}, u^{\prime \prime}: v_{K}=u^{\prime}+u^{\prime \prime}, v^{\prime}, v^{\prime \prime}: v_{L}=v^{\prime}+v^{\prime \prime}, u w^{\prime}, w^{\prime \prime}: v_{M}=w^{\prime}+w^{\prime \prime}$, что $\alpha_{L}=-\alpha_{0} u$

i) $\alpha_{L} \cdot v^{\prime}, \alpha_{L} \cdot v^{\prime \prime} \geqslant 0 ; \quad \alpha_{K} \cdot u^{\prime}, \alpha_{K} \cdot u^{\prime \prime} \geqslant 0 ; \quad \alpha_{M} \cdot w^{\prime}, \alpha_{M} \cdot w^{\prime \prime} \geqslant 0 ;$

ii) $\left(\sum_{i=1}^{K-1} v_{i}+u^{\prime}\right)+\left(w^{\prime \prime}+\sum_{i=M+1}^{n} v_{i}\right)=0$;

$$
u^{\prime \prime}+\sum_{i=K+1}^{L-1} v_{i}+v^{\prime}=0 ; \quad v^{\prime \prime}+\sum_{i=L+1}^{M-1} v_{i}+w^{\prime}=0 ;
$$

iii) $S_{n}(V)=S_{L-K}\left(V_{1}\right)+S_{M-L}\left(V_{2}\right)+S_{n}\left(V_{3}\right)$,

гдe

$$
\begin{aligned}
& V_{1}=\left(u^{\prime \prime}, v_{K+1}, \ldots, v_{L-1}, v^{\prime}\right) \\
& V_{2}=\left(v^{\prime \prime}, v_{L+1}, \ldots, v_{M-1}, w^{\prime}\right) \\
& V_{3}=\left(v_{0}, \ldots, v_{K-1}, u^{\prime}, 0_{M-K-1}, w^{\prime \prime}, v_{M+1}, \ldots, v_{n}\right) .
\end{aligned}
$$

По определению индексов $K, M$ и $(3.9)$, ii) $V_{3}$ является простым вектором. 
ЗАмечаниЕ 3.10 . Мы также отметим следуюшее свойство векторов $V_{1}$ и $V_{2}$ (см. рис. 3.2 -і и 3.2 -іі соответственно):

i) $\sum_{i=1}^{K}\left|v_{i}\right| \leqslant \sum_{i=M}^{n}\left|v_{i}\right| \Longrightarrow u^{\prime}=v_{k}, \quad u^{\prime \prime}=0 ;$

ii) $\sum_{i=1}^{K}\left|v_{i}\right| \geqslant \sum_{i=M}^{n}\left|v_{i}\right| \Longrightarrow w^{\prime}=0, \quad w^{\prime \prime}=v_{M}$.

\begin{tabular}{|c|c|c|c|c|c|c|c|c|}
\hline$v_{0}$ & & $v_{K}$ & $\begin{array}{lll}v^{\prime} & v\end{array}$ & $v^{\prime \prime}$ & $w^{\prime} v$ & I $w^{\prime \prime}$ & & $v_{n}$ \\
\hline+ & + & + & + & + & - & - & - & - \\
\hline
\end{tabular}

Рис. 3.2-і. Случай векторов с нечетным числом перемен знака

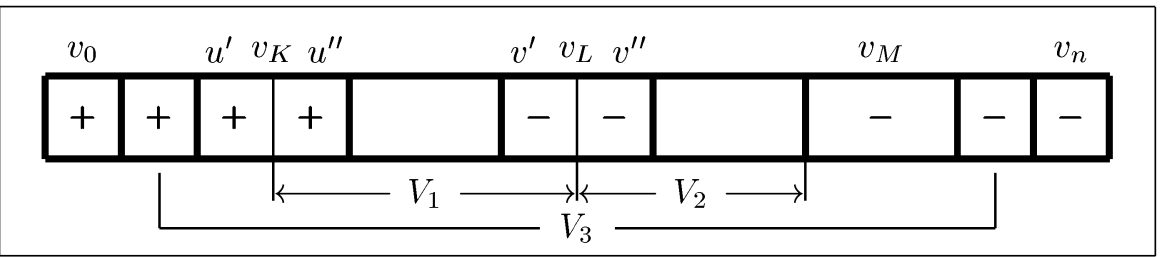

Рис. 3.2-іi. Случай векторов с нечетным числом перемен знака

Индуктивное доказательство теоремы 1.4 проводится так же, как и в п. 3.2. Заметим, что если $\mathscr{M} \neq \varnothing$, то набор простых векторов разложения также содержит простой вектор $V_{3}$. Если $l_{1}, l_{2}, l$ - числа перемен знака векторов $V_{1}, V_{2}, V$ соответственно, то $l=l_{1}+l_{2}$ и по нашему индуктивному предположению число $m$ простых векторов в разложении $V$ оценивается следующим образом:

$$
m \leqslant 1+\sum_{k=1}^{2}\left\lfloor\frac{3 l_{k}-1}{2}\right\rfloor \leqslant\left\lfloor\frac{3\left(l_{1}+l_{2}\right)-1}{2}\right\rfloor=\left\lfloor\frac{3 l-1}{2}\right\rfloor,
$$

так как одно из чисел $l_{1}, l_{2}$ четно, а другое нечетно.

Пусть индексы $K, L, M \in \mathbb{Z}[n]$, числа $u^{\prime}, u^{\prime \prime}: u^{\prime}+u^{\prime \prime}=v_{K}, \quad v^{\prime}, v^{\prime \prime}$ : $v^{\prime}+v^{\prime \prime}=v_{L}, \quad w^{\prime}, w^{\prime \prime}: w^{\prime}+w^{\prime \prime}=v_{M}$, и векторы $\left\{V_{k}\right\}_{k=1}^{3}$ определены в формулировке теоремы 3.10 .

СлЕДСТВИЕ 3.11. Пусть $Z^{*}=\left(z_{0}^{*}, \ldots, z_{n}^{*}\right)$ - әкстремальный вектор задачи (*) и

$$
\begin{gathered}
Z_{1}^{*}:=\left(0, z_{K+1}^{*}-z_{K}^{*}, \ldots, z_{L}^{*}-z_{K}^{*}\right), \quad Z_{2}^{*}:=\left(0, z_{L+1}^{*}-z_{L}^{*}, \ldots, z_{M}^{*}-z_{L}^{*}\right) \\
Z_{3}^{*}:=\left(z_{0}^{*}, \ldots, z_{K}^{*}, 0_{M-K-1}, z_{M}^{*}, \ldots, z_{n}^{*}\right) .
\end{gathered}
$$


Если $Z^{*}$ - единственная әкстремаль задачи (*), то для $k=1,2,3$ вектор $Z_{k}^{*}-$ едиственное решение задачи

$$
\left\langle Z, V_{k}\right\rangle \rightarrow \sup , \quad Z \in \mathbb{H}_{0}^{\omega}\left[n_{k}\right],
$$

и если $V_{3} \neq 0$, то $Z_{3}^{*}$ - единственное решение (3.14) при $k=3$, где $n_{1}=L-K$, $n_{2}=M-L u n_{3}=n$.

ЗАмечАниЕ 3.12 . Если проблема $(*)$ имеет единственное решение, то $v^{\prime} \neq 0$, $v^{\prime \prime} \neq 0$ и либо $u^{\prime} \neq 0, u^{\prime \prime} \neq 0$, либо $w^{\prime} \neq 0, w^{\prime \prime} \neq 0$.

3.4. Задача (**) для векторов $V=\left(v_{0}, \ldots, v_{n}\right) \in \mathbb{R}^{n+1} \backslash \mathbb{R}_{0}^{n+1}$. В этом пункте будет показано, что любая экстремаль задачи $(* *)$ для вектора $V=\left(v_{0}, \ldots, v_{n}\right)$ с $\sum_{i=0}^{n} v_{i} \neq 0$ также является решением $(* *)$ для некоторого вектора $\widetilde{V}=$ $\left(\tilde{v}_{0}, \ldots, \tilde{v}_{n}\right) \mathrm{c} \sum_{i=0}^{n} \tilde{v}_{i}=0$.

ЗАмечание 3.13 . Если $V \in \mathbb{R}_{0}^{n+1}$, то вектор $Z^{*}=\left(z_{0}^{*}, \ldots, z_{n}^{*}\right)$ экстремален в $(*)$ тогда и только тогда, когда вектор $Z_{p}^{*}:=\left(z_{0}^{*}-z_{p}^{*}, z_{1}^{*}-z_{p}^{*}, \ldots, z_{n}^{*}-z_{p}^{*}\right)$ является решением задачи $(*)$. Следовательно, элементы разложения $\left\{v_{i, j}\right\}_{i, j \in \mathbb{Z}[n]}$ вектора $V$, ассоциируемые с задачей $(* *)$, не зависят от индекса $p \in \mathbb{Z}[n]$.

Пусть $\mathbb{S}:=\sum_{i=0}^{n} v_{i} \neq 0$ и $S=\operatorname{sign} \mathbb{S}$. Тогда вектор

$$
\widetilde{V}:=\left(v_{0}, v_{1}, v_{p-1}, v_{p}-\mathbb{S}, v_{p+1}, \ldots, v_{n}\right)
$$

принадлежит $\mathbb{R}_{0}^{n+1}$. Пусть $\left\{\tilde{v}_{i, j}\right\}_{i, j \in \mathbb{Z}[n]}$ - разложение вектора $\widetilde{V}$, ассоциируемое с задачей

$$
\langle\widetilde{V}, Z\rangle \rightarrow \sup , \quad Z \in \mathbb{H}_{p}^{\omega}[n] .
$$

Тогда $V$ допускает следуюшее разложение $\left\{v_{i, j}\right\}_{i, j \in \mathbb{Z}[n]},\left\{\hat{v}_{i}\right\}_{i=0}^{n}$, описанное в теореме 1.2 .

Если $S \cdot\left(\alpha_{p}-\mathbb{S}\right) \geqslant 0$, то

$$
\begin{array}{rlrl}
v_{i, j} & =\tilde{v}_{i, j}, & i, j & \in \mathbb{Z}[n] ; \\
\hat{v}_{p} & =S, \quad \hat{v}_{i}=0, \quad i \in \mathbb{Z}[n] \backslash\{p\} .
\end{array}
$$

Если $S \cdot\left(\alpha_{p}-\mathbb{S}\right)<0$, то

$$
\begin{aligned}
& v_{i, j}=\tilde{v}_{i, j}, \quad v_{p, i}=v_{i, p}=0, \quad i, j \in \mathbb{Z}[n] ; \\
& \hat{v}_{p}=v_{p}, \quad \hat{v}_{j}=\tilde{v}_{j, p}, \quad j \in \mathbb{Z}[n] \backslash\{p\} .
\end{aligned}
$$

ЗАмечание 3.14. Экстремальные векторы задач $(* *)$ и (3.15) совпадают.

Замечания 3.13 и 3.14 редуцируют решение задачи $(* *)$ к описанию экстремалей проблемы $(*)$ для векторов $V \in \mathbb{R}_{0}^{n+1}$ с нулевой суммой координат. 


\section{§ 4. Специальные варианты задачи $(*)$}

В этом параграфе мы рассмотрим специальные случаи задачи $(*)$, широко используемые в разнообразных приложениях теории приближений, оптимальном контроле, функциональном анализе, математической экономике и конструктивной теории функций. Мы обсудим некоторые из этих приложений в $\S 6-10$.

В п. 4.1 мы раскроем связь между дискретным вариантом и непрерывной версией задачи $(*)$ о максимизации интегральных функционалов

$$
h \mapsto \int_{a}^{b} h(t) \psi(t) d t
$$

во множестве функций $h \in H^{\omega}[a, b]: h(a)=0$, при условии, что ядра $\psi \in C[a, b]$ имеют конечное число перемен знака на $[a, b]$. В п. 4.2 мы исследуем перестановки целочисленных векторов. Наконец в п. 4.3 мы сведем описание экстремалей задачи

$$
\langle V, Z\rangle \rightarrow \sup , \quad Z=\left(z_{0}, \ldots, z_{n}\right) \in \mathbb{H}_{\omega}[n], \quad z_{0}=\xi_{1}, \quad z_{n}=\xi_{2},
$$

к решению двух непрерывных вариантов проблемы $(*)$.

4.1. Дискретный и непрерывный варианты задачи $(*)$. Пусть $\mathbb{I}=[a, b]$, $-\infty<a<b<\infty$, и $\psi$ - непрерывная функция на $\mathbb{I}$. Мы проведем предельньй переход от дискретной задачи $(*)$ к непрерывной версии

$$
\int_{\mathbb{I}} h(t) \psi(t) d t \rightarrow \sup , \quad h \in H_{a}^{\omega}(\mathbb{I}),
$$

где классы $H_{a}^{\omega}(\mathbb{I})$ были введены в определении 0.4.

Для $n \in \mathbb{N}$ положим $\alpha_{2^{n}}^{n}:=b$ и

$$
\begin{array}{lll}
\alpha_{i}^{n}:=a+2^{-n}(b-a) i, & \mathbb{I}_{i}^{n}:=\left[\alpha_{i}^{n}, \alpha_{i+1}^{n}\right], & 0 \leqslant i \leqslant 2^{n}-1 ; \\
V^{n}=\left(v_{0}^{n}, v_{1}^{n}, \ldots, v_{2^{n}-1}^{n}\right), & v_{i}^{n}:=2^{-n} \psi\left(\alpha_{i}^{n}\right), & 0 \leqslant i \leqslant 2^{n}-1 .
\end{array}
$$

ПРЕДЛОЖЕНИЕ 4.1. Пусть $V_{n}$ определен в (4.2) для $n \in \mathbb{N}$. Тогда

$$
\mathscr{A}:=\sup \left\{\int_{\mathbb{I}} h(t) \psi(t) d t \mid h \in H_{a}^{\omega}(\mathbb{I})\right\}=\lim _{n \rightarrow \infty} \sup _{Z \in \mathbb{H}_{0}^{\omega}\left[2^{n}-1\right]}\left\langle V^{n}, Z\right\rangle=: \mathscr{B} .
$$


ДокАЗАТЕЛЬСТво. Если $h \in H_{a}^{\omega}(\mathbb{I})$, то вектор $Z^{n}=\left(z_{0}^{n}, z_{1}^{n}, \ldots, z_{2^{n}-1}^{n}\right)$ с координатами

$$
z_{i}^{n}:=h\left(\alpha_{i}^{n}\right), \quad i=0, \ldots, 2^{n}-1,
$$

принадлежит $\mathbb{H}_{0}^{\omega}\left[2^{n}-1\right]$ и

$$
\begin{aligned}
& \left|\int_{\mathbb{I}} h(t) \psi(t) d t-\left\langle V^{n}, Z^{n}\right\rangle\right| \leqslant \sum_{i=0}^{2^{n}-1} \int_{\mathbb{I}_{i}}\left|\psi(t)-\psi\left(\alpha_{i}^{n}\right)\right| \cdot|h(t)| d t \\
& \quad+\sum_{i=0}^{2^{n}-1} \int_{\mathbb{I}_{i}}\left|h(t)-h\left(\alpha_{i}^{n}\right)\right| \cdot\left|\psi\left(\alpha_{i}^{n}\right)\right| d t \\
& \leqslant|\mathbb{I}| \cdot\left[\omega\left(\psi ; 2^{-n}\right) \cdot \omega(|\mathbb{I}|)+\omega\left(2^{-n}\right)\|\psi\|_{C(\mathbb{I})}\right] .
\end{aligned}
$$

Таким образом, мы имеем неравенство $\mathscr{A} \leqslant \mathscr{B}$. С другой стороны, если $Z^{n}=$ $\left(z_{0}^{n}, \ldots, z_{2^{n}-1}^{n}\right)$ принадлежит $\mathbb{H}_{0}^{\omega}\left[2^{n}-1\right]$, то кусочно линейная функция

$$
h(x):=z_{i-1}^{n}+\left(z_{i}^{n}-z_{i-1}^{n}\right) \frac{x-\alpha_{i-1}^{n}}{\alpha_{i}^{n}-\alpha_{i-1}^{n}}, \quad x \in\left[\alpha_{i-1}^{n}, \alpha_{i}^{n}\right], \quad i=1, \ldots, 2^{n}-1,
$$

принадлежит $H_{a}^{\omega}\left[0,2^{n}-1\right]$ и $Z^{n}$ - ограничение $h$ на множество точек $\left\{\alpha_{i}^{n}\right\}_{i=0}^{2^{n}-1}$. Тогда то же неравенство (4.5) дает нам возможность заключить, что $\mathscr{B} \leqslant \mathscr{A}$.

4.2. Перестановки целочисленных векторов. Множество экстремалей задачи $(*)$ является выпуклым множеством в $\mathbb{R}^{n+1}$. Аналогично, если существуют два множества элементов $\left\{v_{i, j}^{k}\right\}_{i, j \in \mathcal{N}^{n}}, k=1,2$, разложения вектора $V$, то $\left\{\alpha v_{i, j}^{1}+(1-\alpha) v_{i, j}^{2}\right\}_{i, j \in \mathcal{N}^{n}}-$ декомпозиция $V$ для любого $\alpha \in(0,1)$. Таким образом, множество перестановок $\Lambda_{\omega}(V)$ вектора $V$, определенных свойством $S_{n}(V)=\left\langle V, \Omega_{n}\right\rangle\left(\Omega_{n}=(\omega(0), \omega(1), \ldots, \omega(n))\right)$, выпукло в $\mathbb{R}^{n+1}$.

ПримеР 4.2. Пусть $V=\left(v_{0}, \ldots, v_{18}\right)$ - вектор в $\mathbb{R}^{19}$ с ненулевыми координатами $v_{0}=1, v_{8}=-2, v_{10}=2, v_{18}=-1$ и $\omega(t)=\omega_{\alpha}(t)=t^{\alpha}, 0<\alpha \leqslant 1$. Перечислим ненулевые элементы $v_{i, j}$ разложения (1.1) для $0 \leqslant i<j \leqslant n$, ненулевые координаты перестановки $\Lambda_{\omega_{\alpha}}(V)=\left(\lambda_{0}, \lambda_{1}, \ldots, \lambda_{18}\right)$ и значение $M_{\alpha}=M_{\omega_{a}}(V)$ максимума $(*)$ при $\omega(t)=t^{\alpha}$.

Случай $0<\alpha<1 / 2: v_{0,18}=\lambda_{18}=1, v_{8,10}=\lambda_{2}=2 ; M_{\alpha}=2^{\alpha+1}+2^{\alpha} 3^{2 \alpha}$.

Случай $1 / 2<\alpha<1: v_{0,8}=v_{8,10}=v_{10,18}=\lambda_{2}=1 ; \lambda_{8}=2 ; M_{\alpha}=2^{\alpha}+2^{3 \alpha+1}$.

Случай $\alpha=1 / 2: v_{0,8}=v_{10,18}=2-\beta ; v_{0,18}=\lambda_{18}=\beta-1 ; v_{8,10}=\lambda_{2}=\beta$; $\lambda_{8}=4-2 \beta$ для всех $\beta \in[1,2]$ и $M_{\alpha}=5 \sqrt{2}$.

Последний пример показывает существование нецелых перестановок $\Lambda_{\omega}(V)$ и элементов разложения у целочисленных векторов $V$. С другой стороны, следующий результат гарантирует существование целочисленных перестановок у целочисленных векторов $V$. 
ПРЕДЛОЖЕНИЕ 4.3. Если $V=\left(v_{0}, v_{1}, \ldots, v_{n}\right) \in \mathbb{Z}^{n+1}$, то существует перестановка $\Lambda_{\omega}(V)$ в $\mathbb{Z}_{+}^{n+1}$.

ДокАЗАТЕЛЬСТво. Результат будет доказан индукцией по числу перемен знака вектора $V$. Ввиду замечания 2.4 предложение справедливо для простых векторов $V$. В дальнейшем анализе мы опираемся на результаты пп. 3.1, 3.2.

Если $V$ имеет четное число перемен знака, то согласно $(3.1)$, ii) $v^{\prime}=-\sum_{i=1}^{L-1} v_{i}$ и $v^{\prime \prime}=-\sum_{i=L+1}^{n} v_{i}$. Таким образом, $V_{1} \in \mathbb{Z}^{L+1}$ и $V_{2} \in \mathbb{Z}^{n-L+1}$. Следовательно, по индуктивному предположению существуют целочисленные перестановки $\Lambda_{\omega}\left(V_{1}\right)$ и $\Lambda_{\omega}\left(V_{2}\right)$. Тогда перестановка $\Lambda_{\omega}(V)=\left(\Lambda_{\omega}\left(V_{1}\right), 0_{n-L}\right)+\left(\Lambda_{\omega}\left(V_{2}\right), 0_{L}\right)$ также имеет целочисленные координаты.

Если $V$ имеет нечетное число перемен знака, то по теореме 3.9 и замечанию $3.10 u^{\prime}, u^{\prime \prime}, v^{\prime}, v^{\prime \prime}, w^{\prime}, w^{\prime \prime}$ - целые числа. Следовательно, $V_{1} \in \mathbb{Z}^{L-K+1}$, $V_{2} \in \mathbb{Z}^{M-L+1}$ и $V_{3} \in \mathbb{Z}^{n+1}$. По нашему индуктивному допущению существуют $\Lambda_{\omega}\left(V_{1}\right)$ в $\mathbb{Z}_{+}^{L-K+1}, \quad \Lambda_{\omega}\left(V_{2}\right)$ в $\mathbb{Z}_{+}^{M-L+1}$ и $\Lambda_{\omega}\left(V_{3}\right)$ в $\mathbb{Z}_{+}^{n+1}$. Тогда $\Lambda_{\omega}(V):=$ $\left(\Lambda_{\omega}\left(V_{1}\right), 0_{n-L+K}\right)+\left(\Lambda_{\omega}\left(V_{2}\right), 0_{n-L+M}\right)+\Lambda_{\omega}\left(V_{3}\right)$ имеет целочисленные компоненты.

4.3. Задача с фиксированными граничными условиями. Рассмотрим проблему

$$
\langle V, Z\rangle \rightarrow \sup , \quad Z=\left(z_{0}, \ldots, z_{n}\right) \in \mathbb{H}_{\omega}[n], \quad z_{0}=\xi_{1}, \quad z_{n}=\xi_{2}
$$

для таких $\xi_{1}, \xi_{2} \in \mathbb{R}$, что $\left|\xi_{2}-\xi_{1}\right| \leqslant \omega(n)$.

Следуюший результат и замечание о структуре экстремалей задачи (4.7) устанавливаются теми же рассуждениями, что и теоремы 3.6 и 3.9 в $\S 3$.

TEOPEMA 4.4. Пусть $V=\left(v_{0}, \ldots, v_{n}\right)$. Если $\left|\xi_{1}-\xi_{2}\right| \leqslant \omega(n)$, то существуют индекс $L \in(0, n)$ и такие $v^{\prime}, v^{\prime \prime}$, что:

$$
\begin{aligned}
& \text { i) } \alpha_{L} v^{\prime} \geqslant 0, \quad \alpha_{L} v^{\prime \prime} \geqslant 0, \quad v^{\prime}+v^{\prime \prime}=v_{L} ; \\
& \text { ii) } \sup \left\{\langle Z, V\rangle \mid Z \in \mathbb{H}^{\omega}[n], z_{0}=\xi_{1}, z_{n}=\xi_{2}\right\} \\
& =\sup \left\{\left\langle Z, V^{1}\right\rangle \mid Z \in \mathbb{H}^{\omega}[L], z_{0}=\xi_{1}\right\} \\
& \quad+\sup \left\{\left\langle Z, V^{2}\right\rangle \mid Z \in \mathbb{H}^{\omega}[n-L], z_{0}=\xi_{2}\right\},
\end{aligned}
$$

əде

$$
V^{1}:=\left(v_{0}, v_{1}, \ldots, v_{L-1}, v^{\prime}\right), \quad V^{2}:=\left(v_{n}, v_{n-1}, \ldots, v_{L+1}, v^{\prime \prime}\right) .
$$

Пусть $Z^{*}=\left(z_{0}^{*}, \ldots, z_{n}^{*}\right)$ - экстремальньй вектор в $(4.7)$,

$$
\begin{aligned}
& A:=\max \left\{k \in \mathbb{Z}[n]|| z_{k}-z_{0}|=| z_{k}-\xi_{1} \mid=\omega(k)\right\}, \\
& B:=\min \left\{k \in \mathbb{Z}[n]|| z_{n}-z_{k}|=| \xi_{2}-z_{k} \mid=\omega(n-k)\right\},
\end{aligned}
$$

и $\left\{c_{i}\right\}_{i=0}^{k}, A=: c_{0}<c_{1}<\cdots<c_{k-1}<c_{k}:=B,-$ максимальная система индексов со свойствами

$$
\left|z_{c_{i}}^{*}-z_{c_{i-1}}^{*}\right|=\omega\left(c_{i}-c_{i-1}\right), \quad i=1, \ldots, k \text {. }
$$


Набор $\left\{c_{i}\right\}_{i=0}^{k}$ исчерпывает множество индексов $L$, наделенных свойством (4.8), ii). Если множество $\left\{c_{i}\right\}_{i=1}^{k-1}$ непусто, то координаты вектора $V$ меняют знак по меньшей мере один раз на $\left[c_{i-1}, c_{i}\right]$. Следовательно, число точек со свойством $\left.(4.8), \mathrm{ii}\right)$ может превосходить число перемен знака $V$ лишь на единицу.

\section{§ 5. Приложение к теории графов}

В этом параграфе мы введем понятие графа перестановок $\mathbb{G}_{\omega}(V)$ и исследуем свойства отображения $V \mapsto \mathbb{G}_{\omega}(V)$ из пространства $\mathbb{R}_{0}^{n+1}$ во множество графов с вершинами в $\mathbb{Z}[n]$ и ребрами $\mathbb{Z}[n] \times \mathbb{Z}[n]$. Структурные особенности этих графов $\mathbb{G}_{\omega}(V)$ проливают новый свет на свойства решений задачи $(*)$, установленных в $\S 1-4$.

5.1. Определение, геометрическая реализация графов перестановок. Основная терминология и результаты, используемые в этом параграфе, приведены в [11], [60] или любой другой книге по комбинаторике или теории графов.

ОПРЕДЕЛЕНИЕ 5.1. Пусть $V \in \mathbb{R}_{0}^{n+1}, \omega$ - строго выпуклый модуль непрерьвности и $\left\{v_{i, j}\right\}_{i, j \in \mathbb{Z}[n]}$ - множество элементов разложения вектора $V$, ассоциируемых с задачей $(*)$. Тогда граф $\mathbb{G}_{\omega}(V)=(\mathbb{E}, \mathbb{V})$ с множествами ребер $\mathbb{E} \subset \mathbb{Z}[n]$ и вершин $\mathbb{V} \subset \mathbb{Z}[n] \times \mathbb{Z}[n]$ определяется посредством матрицы смежсности $\mathscr{I}=$ $\left\{I_{i, j}\right\}_{i, j \in \mathbb{Z}[n]}:$

$$
I_{i, j}:=\operatorname{sign}\left|v_{i, j}\right|, \quad i, j \in \mathbb{Z}[n] .
$$

Другими словами, две вершины $i, j$ графа $\mathbb{G}_{\omega}(V)$ соединены вершиной тогда и только тогда, когда $v_{i, j} \neq 0$.

ЗАмЕчАниЕ 5.2. Если задача (*) имеет континуум решений, то возможно существование графов $\mathbb{G}_{\omega}(V)$, порожденных разными элементами $\left\{v_{i, j}\right\}_{i, j \in \mathbb{Z}[n]}$ декомпозиции вектора $V \in \mathbb{R}_{0}^{n+1}$.

В нашем анализе графов перестановок мы используем следуюшее следствие леммы 3.5 .

ЛЕмма 5.3. Если $v_{i, j} \neq 0 u v_{l, k} \neq 0$, то выполняется одно из следуюших условий:

$$
[i, j] \subseteq[l, k], \quad[l, k] \subset[i, j], \quad(i, j) \cap[l, k]=\varnothing
$$

ОПРЕДЕЛЕНИЕ 5.4. Пусть $A=\left\{a_{i, j}\right\}_{i, j \in \mathbb{Z}[n]}$ и $0 \leqslant i_{1}<i_{2} \leqslant n, 0 \leqslant j_{1}<$ $j_{2} \leqslant n$. Тогда $A\left[i_{1}, i_{2} ; j_{1}, j_{2}\right]=\left\{\alpha_{i, j}\right\}_{\substack{0 \leqslant i \leqslant i_{2}-i_{1} \\ 0 \leqslant j \leqslant j_{2}-j_{1}}}$ обозначает ограничение матрицы $A$ на строки $i_{1}, \ldots, i_{2}$ и столбцы $j_{1}, \ldots, j_{2}$ :

$$
\alpha_{i, j}:=a_{i+i_{1}, j+j_{1}}, \quad 0 \leqslant i \leqslant i_{2}-i_{1}, \quad 0 \leqslant j \leqslant j_{2}-j_{1} .
$$

Теперь лемма 5.3 может быть переформулирована в терминах миноров матрицы смежности $\mathscr{I}$. 
Лемма 5.5. Если $I_{i, j}=1$, mo

$$
\begin{aligned}
\mathscr{I}[i+1, j-1 ; 0, i-1] & =\mathbf{O}, & \mathscr{I}[i+1, j-1 ; j+1, n] & =\mathbf{O}, \\
\mathscr{I}[0, i-1 ; i+1, j-1] & =\mathbf{O}, & & \mathscr{I}[j+1, n ; i+1, j-1]=\mathbf{O},
\end{aligned}
$$

где $\mathscr{I}=\left\{I_{i, j}\right\}_{i, j \in \mathbb{Z}[n]}-$ матрица смежности $\mathbb{G}_{\omega}(V)$ и $\mathbf{O}-$ нулевые матрицы соответствующих размерностей.

ПРЕДЛОЖЕНИЕ 5.6. Любой граф $\mathbb{G}_{\omega}(V)$ перестановок является двудольным.м планарныцм графом.

ДокАЗАТЕльСтво. Во-первых, если $v_{i}=0$, то вершина $i$ изолирована. Граф $\mathbb{G}_{\omega}(V)$ не имеет петель, поскольку $v_{i, i}=0$ для всех $i \in \mathbb{Z}[n]$. Положим $V_{+}:=$ $\left\{i \in \mathbb{Z}[n]: v_{i} \geqslant 0\right\}$ и $V_{-}:=\left\{i \in \mathbb{Z}[n]: v_{i}<0\right\}$. Поскольку $v_{i, j} \neq 0$, только если $v_{i} \cdot v_{j}<0$, множества $V_{+}$и $V_{-}$дают желаемое разбиение $\mathbb{V}(G)$. Факт двудольности графа $\mathbb{G}_{\omega}(V)$ также легко вытекает из критерия Кенига, поскольку любой цикл нечетной длины в $\mathbb{G}_{\omega}(V)$ обязательно содержал бы две соседние вершины $i$ и $j$ c $v_{i} \cdot v_{j}>0$, что невозможно, поскольку такие вершины разъединены.

Во-вторых, основываясь на (5.2), мы можем предложить следующую планарную геометрическую реализацию графа $\mathbb{G}_{\omega}(V)$ на декартовой плоскости. Для $i, j \in \mathbb{Z}[n]$ представим вершину $i$ точкой $(i, 0)$, а жорданову кривую $e_{i, j}$, соединяющую точки $(i, 0)$ и $(j, 0)$, в случае $(i, j) \in \mathbb{E}\left(\mathbb{G}_{\omega}(V)\right)\left(v_{i, j} \neq 0\right)$ - планарной аркой окружности радиуса $R>\max \left\{j-i \mid v_{i, j} \neq 0\right\}$, лежащей в верхней полуплоскости и имеющей центр в нижней полуплоскости (см. рис. 5.1).

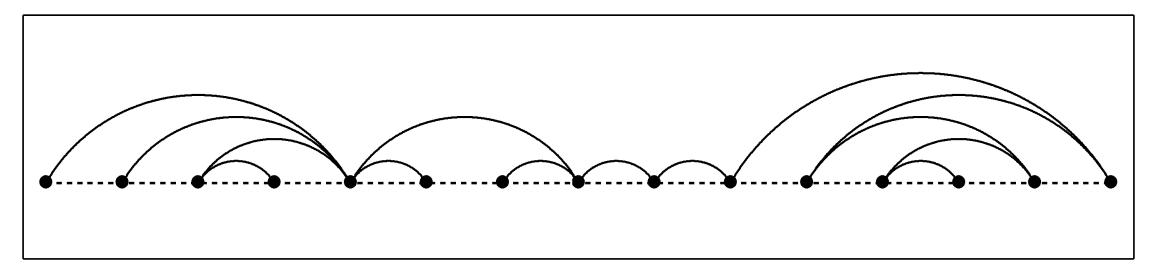

Рис. 5.1. Планарная реализация графа перестановок $\mathbb{G}_{\omega}(\mathbb{V})$

Лемма 5.3 гарантирует, что любые две арки $e_{i_{1}, j_{1}}, e_{i_{2}, j_{2}}$ могут пересекаться только в вершинах графа.

5.2. Планарное представление $\mathbb{G}_{\omega}(V)$ для простых векторов. Результаты $\S 2$ дают возможность получить естественное планарное представление графов $\mathbb{G}_{\omega}(V)$ для простых $V$.

ПРЕДЛОЖЕНИЕ 5.7. Если $V$ - простой вектор, то граф $\mathbb{G}_{\omega}(V)$ допускает планарную реализацию, в которой все ребра представлены прямолинейными сегментами. 
ДокАЗАТЕЛЬСтво. Вершины графа $\mathbb{G}_{\omega}(V)$ поставлены в соответствие точкам на декартовой плоскости согласно правилу

$$
i \mapsto\left(\stackrel{\circ}{v}_{i}, \alpha_{i}+\left(1-\left|\alpha_{i}\right|\right)(i+2)\right), \quad i \in \mathbb{Z}[n],
$$

где $\stackrel{\circ}{v}_{i}=\sum_{j=0}^{i} v_{j}$ и $\alpha_{i}=\operatorname{sign} v_{i}, i=0, \ldots, n$. Заметим, что изолированная вершина $i \quad\left(v_{i}=0\right)$ лежит в области $\left\{(x, y) \in \mathbb{R}^{2}|| y \mid \geqslant 2\right\}$. Если $v_{i} \neq 0$, то $i$ лежит на прямой $y=\operatorname{sign} v_{i}$. Множество $\mathbb{E}$ ребер состоит из пар точек на плоскости, соответствуюших вершинам

$$
\left(i, j_{i}\right), \quad j_{i}:=\min \left\{\stackrel{\circ}{v}_{k}(V) \mid m \leqslant k \leqslant n, \stackrel{\circ}{v}_{k}(V)>\stackrel{\circ}{v}_{i}(V), \alpha_{k} \cdot \alpha_{i}=-1\right\},
$$

соединенных прямолинейными сегментами (см. рис. 5.2).

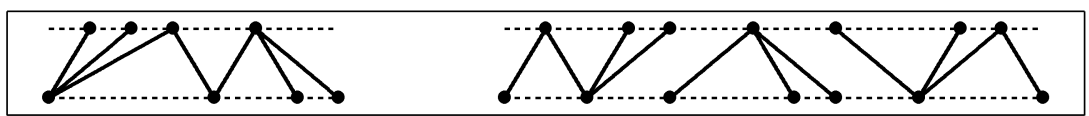

Рис. 5.2. Планарная реализация графа $\mathbb{G} \omega(V)$ для простого вектора $V$

Заметим, что граф $\mathbb{G}_{\omega}(V)$ несвязен тогда и только тогда, когда существуют такие индексы $i, j \in \mathbb{Z}[n]: i \neq j$, что $\stackrel{\circ}{v}_{i} \neq \stackrel{\circ}{v}_{j}$. Ввиду предложения 2.1 это означает, что граф $\mathbb{G}_{\omega}(V)$ перестановок для простого $V$ связен тогда и только тогда, когда задача (*) допускает единственное решение. Это наблюдение будет обобщено в теореме 5.13 для всех $V \in \mathbb{R}_{0}^{n+1}$.

5.3. Графическая интерпретация теоремы 3.6. Пусть $V$ - вектор с четньм числом перемен знака и векторы $V_{1}, V_{2}$ определены соотношениями (3.1). Пусть $\widetilde{\mathbb{G}}_{1}(V)=\left(\widetilde{\mathbb{V}}_{1}, \widetilde{\mathbb{E}}_{1}\right)$ и $\widetilde{\mathbb{G}}_{2}(V)=\left(\widetilde{\mathbb{V}}_{2}, \widetilde{\mathbb{E}}_{2}\right)$ - графы перестановок для векторов $\left(V_{1}, 0_{n-L}\right)$ и $\left(0_{L}, V_{2}\right)$ соответственно. Заметим, что $\{i\}_{i=L+1}^{n}$ и $\{i\}_{i=0}^{L-1}-$ изолированные вершины графов $\mathbb{G}_{\omega}\left(V_{1}\right)$ и $\mathbb{G}_{\omega}\left(V_{2}\right)$ соответственно. Введем графы $\mathbb{G}_{1}(V)=\left(\mathbb{V}_{1}, \mathbb{E}_{1}\right)$ и $\mathbb{G}_{2}(V)=\left(\mathbb{V}_{2}, \mathbb{E}_{2}\right)$ :

$$
\mathbb{V}_{1}:=\{0, \ldots, L\}, \quad \mathbb{E}_{1}:=\widetilde{\mathbb{E}}_{1} ; \quad \mathbb{V}_{2}:=\{L, \ldots, n\}, \quad \mathbb{E}_{2}:=\widetilde{\mathbb{E}}_{2}
$$

Теорема 3.6 гарантирует, что $\mathbb{G}_{\omega}(V)$ - граф с вершинами $\mathbb{V}=\{0, \ldots, n\}$ и ребрами $\mathbb{E}=\mathbb{E}_{1} \cup \mathbb{E}_{2}$. Другими словами, $\mathbb{G}_{\omega}(V)=\mathbb{G}_{1}(V) \cup \mathbb{G}_{2}(V)$. Если $v^{\prime} \neq 0$ и $v^{\prime \prime} \neq 0$, то вершина $L$ является точкой сочленения графа $\mathbb{G}_{\omega}(V)$. Если $v^{\prime}=0$ или $v^{\prime \prime}=0$, то компоненты $\mathbb{G}_{1}(V)$ и $\mathbb{G}_{2}(V)$ разъединены в том смысле, что они не имеют общей вершины, инцидентной ребрам в обоих графах $\mathbb{G}_{1}(V)$ и $\mathbb{G}_{2}(V)$. 
5.4. Графическая интерпретация теоремы 3.9. Для $k=1,2,3$ пусть $\mathbb{G}_{k}(V)=\left(\widetilde{\mathbb{V}}_{k}, \widetilde{\mathbb{E}}_{k}\right)-$ графы перестановок векторов $V_{1}=\left(0_{K}, V_{1}, 0_{n-L}\right), \quad V_{2}=$ $\left(0_{L}, V_{2}, 0_{n-M}\right)$ и $V_{3}$ определен (3.9). Введем графы $\mathbb{G}_{k}(V)=\left(\mathbb{V}_{k}, \mathbb{E}_{k}\right), k=1,2,3$ :

$$
\begin{gathered}
\mathbb{V}_{1}:=\{K, \ldots, L\}, \quad \mathbb{V}_{2}:=\{L, \ldots, M\}, \quad \mathbb{V}_{3}:=\{0, \ldots, K\} \cup\{M, \ldots, n\} ; \\
\mathbb{E}_{k}:=\widetilde{\mathbb{E}}_{k}, \quad k=1,2,3 .
\end{gathered}
$$

Теорема 3.9 гарантирует, что $\mathbb{G}_{\omega}(V)=\bigcup_{k=1}^{3} \mathbb{G}_{k}(V)$. Если $v^{\prime} \neq 0$ и $v^{\prime \prime} \neq 0$, то ввиду замечания 3.10 вершина $L$ является точкой сочленения графа $\mathbb{G}_{\omega}(V)$. В противном случае компоненты $\mathbb{G}_{1}(V)$ и $\mathbb{G}_{2}(V)$ разъединены. Аналогично, если $u^{\prime} \neq 0$ и $u^{\prime \prime} \neq 0$, то вершина $K$ - точка сочленения графа $\mathbb{G}_{\omega}(V)$. Иначе $\mathbb{G}_{1}(V)$ и $\mathbb{G}_{3}(V)$ разъединены. Наконец, если $w^{\prime} \neq 0$ и $w^{\prime \prime} \neq 0$, то $M$ - точка сочленения в $\mathbb{G}_{\omega}(V)$. В противном случае $\mathbb{G}_{2}(V)$ и $\mathbb{G}_{3}(V)$ не связаны.

Разложения пा. 5.3, 5.4 сушественным образом используются в индуктивном выводе разнообразных свойств графов перестановок, представленных в следующих двух пунктах.

\section{5. Элементарные свойства графов перестановок.}

ОПРЕдЕЛЕНИЕ 5.8. Цикл (или цепь) графа называется гамильтоновым (әйлеровым), если он проходит через все вершины (ребра). Граф называется гамильтоновым.м (или эйлеровым. ) при условии наличия в нем соответствующего цикла.

ТЕорема 5.9 (Эйлер). Связный граф является әйлеровым, когда степени всех его вершин четны.

ПРЕДЛОЖЕНИЕ 5.10. Связный граф $\mathbb{G}_{\omega}(V)$ не является ни гамильтоновыц, ни эйлеровым.

ДокАЗАТЕЛЬСтво. Граф перестанвок $\mathbb{G}_{\omega}(V)$ не может быть гамильтоновым, поскольку он содержит по крайней мере одну точку сочленения $L$.

Покажем при помоши индуктивного аргумента, что $\mathbb{G}_{\omega}(V)$ не содержит цикла, проходящего через все ребра. Ввиду критерия Эйлера $\mathbb{G}_{\omega}(V)$ не может быть эйлеровым в случае простых векторов $V=\left(v_{0}, \ldots, v_{n}\right)$. Действительно, в этом случае $v_{0, n}=\min \left\{\left|v_{0}\right|,\left|v_{n}\right|\right\}$ и вершина $i=0$ имеет степень 1 , если $\left|v_{0}\right|<\left|v_{n}\right|$. В противном случае степень вершины $i=n$ равна 1 .

Если $\operatorname{sign} v_{0}=\operatorname{sign} v_{n}$ или $\operatorname{sign} v_{0}=-\operatorname{sign} v_{n}$ в случае $\left.3.3,1\right)$, то $\mathbb{G}_{\omega}(V)$ является объединением двух подграфов $\mathbb{G}_{1}(V)$ и $\mathbb{G}_{2}(V)$, определенных в $(5.6)$ или $(5.7)$. Никакие вершины этих двух подграфов не соединены ребром, и точка сочленения $L$ является единственной вершиной, инцидентной ребрам в обоих подграфах $\mathbb{G}_{\omega}\left(V_{1}\right)$ и $\mathbb{G}_{\omega}\left(V_{2}\right)$. Следовательно, любой эйлеров цикл $C$ в графе $\mathbb{G}_{\omega}(V)$ является объединением эйлеровых циклов графов $\mathbb{G}_{\omega}\left(V_{1}\right)$ и $\mathbb{G}_{\omega}\left(V_{2}\right)$. Однако согласно индуктивному допушению ни один из этих графов не имеет эйлерова цикла.

Если $\operatorname{sign} v_{0}=-\operatorname{sign} v_{n}$ и вектор $V_{3}$ является ненулевым простым вектором в случае $3.3,2)$, то или $i=0$, или $i=n$ является вершиной степени 1 , что невозможно для эйлерова графа. 
ОПРЕДЕЛЕНИЕ 5.11. Для $l \geqslant 2 P_{l}=(\mathbb{V}, \mathbb{E})$ определяется как граф с множеством вершин $\mathbb{V}=\mathbb{Z}[l]$ и ребер $\mathbb{E}=\{(k-1, k) \mid k=1, \ldots, l\}$.

Любая гамильтонова цепь в графе $G$ с $l+1$ вершиной может рассматриваться как подграфф, изоморфный $P_{l}$.

ПРЕДЛОЖЕНИЕ 5.12. Связный граф $\mathbb{G}_{\omega}(V)$ перестановок имеет гамильтонову чепь тогда и только тогда, когда он изоморфен $P_{n}$.

ДокаЗАТЕЛЬСтво. Результат легко проверяется в случае простого вектора $V$. В случае $\operatorname{sign} v_{0}=\operatorname{sign} v_{n}$ или в случае $\left.3.3,1\right)$ пусть $L$ - точка сочленения для графов $\mathbb{G}_{1}(V)$ и $\mathbb{G}_{2}(V)$. Тогда любая гамильтонова цепь $H$ в $\mathbb{G}_{\omega}(V)$ распадается на две гамильтоновы цепи $H_{1}$ в $\mathbb{G}_{1}(V)$ и $H_{2}$ в $\mathbb{G}_{2}(V)$, поскольку $L$ - единственная обшая вершина двух графов и никакие другие две вершины этих графов не соединены ребром. Следовательно, по нашему индуктивному допушению мы имеем изоморфизмы $H_{1} \sim P_{L}$ и $H_{2} \sim P_{n-L}$. Более того, $L-$ конечная вершина степени 1 для обеих цепей $H_{1}$ и $H_{2}$. Таким образом, $H \sim P_{n}$. Случай $\left.3.3,2\right) \operatorname{sign} v_{0}=-\operatorname{sign} v_{n}$ рассматривается аналогично.

Рис. 5.3 дает примеры графов $\mathbb{G}_{\omega}(V)$ перестановок с гамильтоновой цепью для векторов $V_{1}=(1,-2,2,-2,2,-1)$ и $V_{2}=(-2,-2,1,2,-2,-1,2,2,-2,2)$ и произвольного $\omega$.

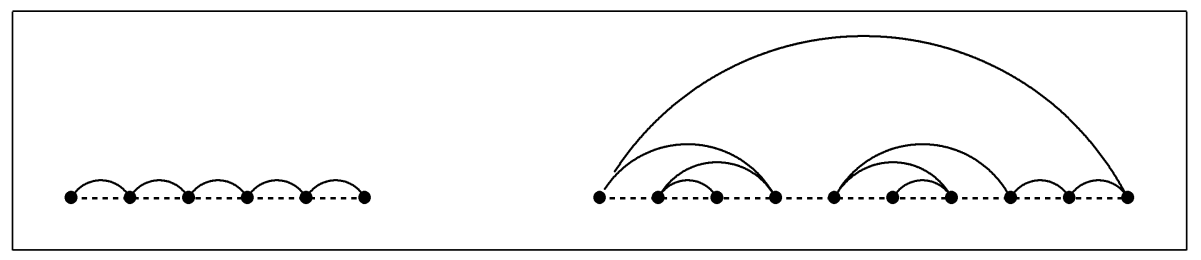

Рис. 5.3. Графы перестановок $\mathbb{G} \omega(V)$ с гамильтоновой цепью

5.6. Связность графов перестановок. В данном пункте мы свяжем вопрос о единственности экстремального вектора в задаче $(*)$ со свойством связности графа $\mathbb{G}_{\omega}(V)$ перестановок.

Теорема 5.13. Задача (*) имеет единственное решение тогда и только тогда, когда граф $\mathbb{G}_{\omega}(V)$ связен.

ДоКАЗАТЕЛЬСТво. Если $v_{i}=0$, то $i-$ изолированная вершина и согласно следствию 3.3 задача $(*)$ имеет континуум экстремалей. Таким образом, мы можем рассматривать только векторы $V$ с ненулевыми координатами. Как обычно, доказательство будет проведено индукцией по числу точек перемен знака вектора $V$.

Результаты п. 5.2 демонстрируют, что критерий единственности решений задачи $(*)$ справедлив для векторов с одной точкой перемены знака. Предположим, что результат доказан для векторов из $\mathbb{R}_{0}^{l}$ при всех $l \leqslant n$. 
Пусть $V=\left(v_{0}, \ldots, v_{n}\right) \in \mathbb{R}_{0}^{n+1}$. Если $\operatorname{sign} v_{0}=\operatorname{sign} v_{n}$, пусть графы $\mathbb{G}_{1}(V)$ и $\mathbb{G}_{2}(V)$ определены в $(5.6)$. Ввиду следствия 3.7 задача $(*)$ имеет единственное решение тогда и только тогда, когда она однозначно разрешима для векторов $V_{1}$ и $V_{2}$. По определению $\mathbb{G}_{1}(V)$ и $\mathbb{G}_{2}(V)$ и нашему индуктивному допушению это требование эквивалентно связности подграфов $\mathbb{G}_{1}(V)$ и $\mathbb{G}_{2}(V)$ и, следовательно, связности графа $\mathbb{G}_{\omega}(V)=\mathbb{G}_{\omega}\left(V_{1}\right) \cup \mathbb{G}_{\omega}\left(V_{2}\right)$.

В случае $\operatorname{sign} v_{0}=-\operatorname{sign} v_{n}$ мы используем аналогичную аргументацию, основанную на разложении (5.7).

5.7. Деревья как графы перестановок. Следующий результат предлагает естественное представление деревьев в качестве графов перестановок.

ПРЕДЛОЖЕНИЕ 5.14. Для любого дерева $\mathscr{T}$ с $n+1$ вершиной и любого выпуклого модуля непрерывности $\omega$ существует такой вектор $V=\left(v_{0}, \ldots, v_{n}\right) \in$ $\mathbb{R}_{0}^{n+1}$, что граф $\mathbb{G}_{\omega}(V)$ изоморфен $\mathscr{T} u\left|v_{i}\right|=\operatorname{deg}\left(w_{i}\right)$, где $w_{i}-$ вериина в $\mathscr{T}$, соответствующая вершине $i$ графа перестановок $\mathbb{G}_{\omega}(V)$ при всех $i \in \mathbb{Z}[n]$.

ДОКАЗАТЕЛЬСТВО. Доказательство проводится индукцией по числу $n$ вершин дерева $\mathscr{T}$. Утверждение тривиально, если $n=2$. Пусть результат справедлив для всех деревьев с не более чем $n$ вершинами и дерево $\mathscr{T}$ имеет $n+1$ вершину. По лемме о рукопожатиях $\mathscr{T}$ имеет по меньшей мере две вершины степени 1 . Пусть $v$ - одна из таких вершин и $w$ - вершина, инцидентная $v$. В этом случае $\mathscr{T} \backslash\{v\}$ представляет собой дерево с $n$ вершинами, изоморфное графу $\mathbb{G}_{\omega}(\widehat{V})$ для некоторого $\widehat{V}=\left(\hat{v}_{0}, \ldots, \hat{v}_{n-1}\right)$. Кроме того, мы имеем свойство $\left|\hat{v}_{i}\right|=\operatorname{deg}\left(w_{i}\right)$, где $w_{i}-$ вершина в $\mathscr{T}$, соответствуюшая $i$ при этом изоморфизме для всех $i=0, \ldots, n-1$.

Пусть $k \in\{0, \ldots, n-1\}$ будет вершиной в $\mathbb{G}_{\omega}(V)$, соответствующей $w$. Тогда мы определим компоненты вектора $V=\left(v_{0}, \ldots, v_{n}\right)$ посредством

$$
v_{i}:= \begin{cases}\hat{v}_{i}, & i=0, \ldots, k-1, \\ \hat{v}_{k}+\operatorname{sign} \bar{v}_{k}, & i=k, \\ -\operatorname{sign} \hat{v}_{k}, & i=k+1, \\ \hat{v}_{i-1}, & i=k+2, \ldots, n .\end{cases}
$$

Тогда множество $\mathbb{E}$ ребер графа $\mathbb{G}_{\omega}(V)$ состоит из пар

$\{(k, k+1),(i, j),(r+1, s+1),(p, q+1) \mid i<j \leqslant k, k \leqslant r<s, p \leqslant k<k+1 \leqslant q\}$

и граф $\mathbb{G}_{\omega}(V)$ обладает желаемьми свойствами.

\section{8. Циклы, грани и отношение Харди-Литтлвуда-Полиа.}

ОБОЗНАЧЕНИЕ 5.15 . Если $0 \leqslant i_{1}<i_{2}<\cdots<i_{k} \leqslant n$, то $C\left[i_{1}, \ldots, i_{k}\right]$ обозначает цикл $i_{1} \rightarrow i_{2} \rightarrow \cdots \rightarrow i_{k} \rightarrow i_{1}$.

Гранища любой внутренней грани (ограничивающей конечную площадь) графа $\mathbb{G}_{\omega}(V)$ содержит ровно один цикл формы $C\left[i_{1}, \ldots, i_{k}\right]$. В частности, число таких граней совпадает с числом циклов $C\left[i_{1}, \ldots, i_{k}\right]$ без подциклов. Следующий результат является необходимым условием для наличия цикла $C\left[i_{1}, \ldots, i_{k}\right]$ в графе перестановок. 
ПРЕДЛОЖЕНИЕ 5.16. Пусть $0 \leqslant i_{k+1}:=i_{1}<\cdots<i_{k} \leqslant n . \quad$ Если $C\left[i_{1}, \ldots, i_{k}\right]-u$ uкл в $\mathbb{G}_{\omega}(V)$, mo $k=2 l, l \in \mathbb{N}, u$

$$
\sum_{j=1}^{k}(-1)^{j} \omega\left(\left|i_{j+1}-i_{j}\right|\right)=0, \quad k=2 l, \quad l \in \mathbb{N}
$$

ДОКАЗАТЕЛЬСТВО. Если $C\left[i_{1}, \ldots, i_{k}\right]$ - цикл в $\mathbb{G}_{\omega}(V)$, то из определения 5.1 и теоремы 1.2 следует, что знаки $\left\{v_{i_{j}}\right\}_{j=1}^{k}$ альтернируют:

$$
\operatorname{sign} v_{i_{j}}=(-1)^{j} \xi, \quad \operatorname{sign} v_{i_{j}, i_{j+1}}=(-1)^{j} \xi, \quad j=1, \ldots, k,
$$

для некоторых $\xi \in\{ \pm 1\}$. Более того, по теореме 1.2 координаты экстремального вектора $Z^{*}=\left(z_{0}^{*}, \ldots, z_{n}^{*}\right)$ задачи $(*)$ удовлетворяют соотношениям

$$
z_{i_{j+1}}^{*}-z_{i_{j}}^{*}=(-1)^{j+1} \xi \cdot \omega\left(\left|i_{j+1}-i_{j}\right|\right), \quad j=1, \ldots, k,
$$

приводящим к (5.9).

ОПРЕДЕЛЕнИЕ 5.17. Пусть $a=\left(a_{1}, \ldots, a_{l}\right)$ и $b=\left(b_{1}, \ldots, b_{l}\right)$ - два вектора в $\mathbb{R}^{l}$. Через $\bar{a}=\left(\bar{a}_{1}, \ldots, \bar{a}_{l}\right)$ и $\bar{b}=\left(\bar{b}_{1}, \ldots, \bar{b}_{l}\right)$ обозначаем возрастающие перестанов$\kappa и a$ и $b$ соответственно. Тогда $a$ и $b$ связаны соотношением $a \lll b$, если

$$
\sum_{i=1}^{k} \bar{a}_{i} \leqslant \sum_{i=1}^{k} \bar{b}_{i}, \quad k=1, \ldots, l .
$$

Отношение «, вариант отношения Харди-Литтлвуда-Полиа, используется в формулировке следуюшего критерия существования графа перестановок $\mathbb{G}_{\omega}(V)$ с фиксированным циклом $C\left[i_{1}, \ldots, i_{k}\right]$.

ПРЕДЛОЖЕНИЕ 5.18. Пусть $i_{k+1}:=i_{1}<\cdots<i_{k}$ nрu $k=2 l, l \in \mathbb{N}, u$ векторы $a=\left(a_{1}, \ldots, a_{l}\right), \quad b=\left(b_{1}, \ldots, b_{l}\right)$ определены посредством

$$
a_{j}:=i_{2 j}-i_{2 j-1}, \quad b_{j}:=\left|i_{2 j+1}-i_{2 j}\right|, \quad j=1, \ldots, l .
$$

Тогда строго выпуклый модуль непрерывности ш со свойством (5.9) существует тогда и только тогда, когда нарушается отношение $а \lll b$ Харди-Литтлвуда-Полиа, т.е. существует такой индекс $m \in\{1, \ldots, l-1\}$, чmo

$$
\sum_{i=1}^{m} \bar{a}_{i}>\sum_{i=1}^{m} \bar{b}_{i}
$$


ДокАЗАТЕЛЬСТво. Условие (5.9) эквивалентно равенству

$$
\sum_{i=1}^{l} \omega\left(\bar{a}_{i}\right)=\sum_{i=1}^{l} \omega\left(\bar{b}_{i}\right)
$$

ЗАмечАниЕ 5.19. Ввиду равенства $\bar{b}_{l}=i_{2 l}-i_{1}=a_{l}+\sum_{j=1}^{l-1}\left(a_{i}+b_{i}\right)$ неравенство (5.13) нарушается при $m=l$.

НЕоБходимость. Предположим, напротив, что

$$
\sum_{i=1}^{k} \bar{a}_{i} \leqslant \sum_{i=1}^{k} \bar{b}_{i}, \quad k=1, \ldots, l .
$$

Покажем при помощи индукции, что из (5.15) вытекают неравенства

$$
\sum_{i=1}^{k} \omega\left(\bar{b}_{i}\right)-\sum_{i=1}^{k} \omega\left(\bar{a}_{i}\right) \geqslant \omega\left(a_{k}+\sum_{i=1}^{k}\left(b_{i}-a_{i}\right)\right)-\omega\left(a_{k}\right), \quad k=1, \ldots, l,
$$

для всех строго выпуклых модулей непрерывности $\omega$. Тогда ввиду замечания 5.19 и $(5.16)$ при $k=l$ строгое неравенство $\sum_{i=1}^{l} \omega\left(\bar{b}_{i}\right)>\sum_{i=1}^{l} \omega\left(\bar{a}_{i}\right)$ будет противоречить (5.14).

Неравенство (5.10) справедливо для $k=2$, так как (5.14) и замечание 5.19 приводят к неравенствам $b_{1}<a_{1} \leqslant a_{2}<b_{2}$ и строго выпуклые модули непрерывности $\omega$ наделены следуюшим свойством:

$$
\left(\omega\left(x_{1}\right)+\omega\left(x_{2}\right)\right)-\left(\omega\left(y_{1}\right)+\omega\left(y_{2}\right)\right) \leqslant \omega\left(x_{1}+x_{2}-y_{1}\right)-\omega\left(y_{2}\right),
$$

при условии, что $x_{2} \geqslant x_{1} \geqslant 0, y_{2} \geqslant y_{1} \geqslant 0, x_{1} \geqslant y_{1}, x_{1}+x_{2}>y_{1}+y_{2}$. Свойство (5.17) следует из убывания $\omega^{\prime}(\cdot)$ для строго выпуклых $\omega$.

Таким образом, согласно индуктивному предположению и (5.17) мы имеем

$$
\begin{aligned}
\sum_{i=1}^{r+1}\left(\omega\left(\bar{b}_{i}\right)-\omega\left(\bar{a}_{i}\right)\right) & \geqslant \omega\left(\bar{a}_{r}+\sum_{i=1}^{r}\left(\bar{b}_{i}-\bar{a}_{i}\right)\right)-\omega\left(\bar{a}_{r}\right)+\omega\left(\bar{b}_{r+1}\right)-\omega\left(\bar{a}_{r+1}\right) \\
& \geqslant \omega\left(\bar{a}_{r+1}+\sum_{i=1}^{r}\left(\bar{b}_{i}-\bar{a}_{i}\right)\right)-\omega\left(\bar{a}_{r+1}\right)
\end{aligned}
$$

что завершает доказательство необходимости условия (5.13) для выполнения (5.9). 
ДостАТочность. Пусть индекс $m \in\{1, \ldots, l-1\}$ таков, что выполняется свойство (5.13) и

$$
S(\omega):=\sum_{i=1}^{l} \omega\left(\bar{b}_{i}\right)-\sum_{i=1}^{l} \omega\left(\bar{a}_{i}\right)
$$

Для $t \geqslant 0$ положим $\omega_{0}(t):=2 t$ и $\omega_{1}(t)=\min \{t, m\}$. Тогда согласно замечанию 5.19

$$
S\left(\omega_{0}\right)=2 \sum_{i=1}^{l} \bar{b}_{i}-2 \sum_{i=1}^{l} \bar{a}_{i}>2 \bar{b}_{l}-2 \sum_{i=1}^{l} \bar{a}_{i}=2 \sum_{i=1}^{l-1} \bar{b}_{i}>0,
$$

в то время как (5.13) означает, что

$$
S(\omega)=\sum_{i=1}^{m} \bar{b}_{i}+m(l-m)-\sum_{i=1}^{m} \bar{a}_{i}-m(l-m)=\sum_{i=1}^{m} \bar{b}_{i}-\sum_{i=1}^{m} \bar{a}_{i}<0 .
$$

Из (5.19) и (5.20) мы выводим сушествование двух таких строго выпуклых модулей непрерывностей $\omega_{0}^{*}$ и $\omega_{1}^{*}$, что

$$
\omega_{0}^{*}(x)<\omega_{1}^{*}(x), \quad x>0 ; \quad S\left(\omega_{0}^{*}\right)>0, \quad S\left(\omega_{1}^{*}\right)<0 .
$$

Непрерьвная гомотопия $h(s, t):=s \omega_{1}^{*}(t)+(1-s) \omega_{0}^{*}(t), s \in[0,1], t \in \mathbb{R}_{+}$, между $h(0, t)=\omega_{0}^{*}(t)$ и $h(1, t)=\omega_{1}^{*}(t)$ такова, что $\omega_{s}(t):=h(s, t)-$ строго выпукльй модуль непрерывности $s \in(0,1)$. Тогда согласно $(5.21)$ существует $\hat{s} \in(0,1)$ со свойством $S\left(\omega_{\hat{s}}\right)=0$.

\section{§ 6. Приложение к математической экономике: "транспортная задача"}

“Транспортная задача" занимает особое положение в теории линейного программирования ввиду ее тесной связи с разнообразными проблемами бизнеса и экономики. В этом параграфе мы рассмотрим следуший ее вариант: предположим, что однородная продукция производится или потребляется в конечном числе фабрик, расположенных в нашей модели в целочисленных точках положительной полупрямой. Предположим, что известен вектор $V$ спроса и предложения: количество продукции, произведенной в точке $i$, представляется компонентой вектора $v_{i}>0$, нужда потребителя $j$ продукции измеряется координатой $v_{j}<0$, пассивная фабрика $k$ соответствует нулевому $v_{k}$ и стоимость перемещения продукции из точки $i$ в точку $j$ равна $\omega(|j-i|)$ для фиксированного выпуклого модуля непрерывности $\omega$. Предположения о совпадении общего спроса и предложения и конечности участников экономических операций эквивалентно допущению $V \in \mathbb{R}_{0}^{n+1}$ для некоторого $n \in \mathbb{N}$. В “транспортной задаче" ставится вопрос об оптимальном распределении продукции между производителями и потребителями с целью минимизации стоимости транспортных затрат. 
В данном параграфе мы сначала напомним читателю понятие и связь между двойственной и прямой задачами линейного программирования. Затем задача (*) и ее двойственная будут поставлены в канонической форме задачи линейного программирования. При этом будет показано, что “транспортная задача" совпадает с вариантом задачи, двойственной к $(*)$. Это наблюдение позволит нам дать удобную экономическую интерпретацию большинства результатов предыдущих параграфов.

Зафиксируем вектор $V=\left(v_{0}, \ldots, v_{n}\right) \in \mathbb{R}_{0}^{n+1}$.

ЗАмЕчАниЕ 6.1 . В случае $V \in \mathbb{R}_{0}^{n+1}$ задачи (**) и задача

$$
\langle V, Z\rangle \rightarrow \sup , \quad Z \in \mathbb{H}^{\omega}[n]
$$

эквивалентны: если $Z^{* *}$ экстремальна в $(* *)$, то $Z^{\star}=Z^{* *}+C \cdot 1_{n+1}$ экстремальна в $(\star)$ для всех $C \in \mathbb{R}$ и, наоборот, $Z^{* *}=Z^{\star}-z_{p}^{\star} \cdot 1_{n+1}-$ решение задачи $(* *)$ при условии, что $Z^{\star}=\left(z_{0}^{\star}, z_{1}^{\star}, \ldots, z_{n}^{\star}\right)$ экстремальна в $(\star)$.

6.1. Двойственная задача линейного программирования. Пусть $A=$ $\left\{a_{i, j}\right\}_{s \times m}$ - матрища размерности $s \times m, b \in \mathbb{R}^{s}$ и $c \in \mathbb{R}^{m}$. Рассмотрим задачу линейного программирования

$$
\langle b, u\rangle \rightarrow \max , \quad u \in \mathbb{U}(A, c):=\left\{u \in \mathbb{R}^{s} \mid A u \leqslant c\right\} .
$$

Мы будем считать, что $\max \{\langle b, u\rangle \mid u \in \mathbb{U}\}<\infty$. Р. Дорфман, П. Самуэльсон и М. Солоу [43, с. 123] перечисляют пять правил перехода от любой задачи линейного программирования к двойственной. В компактной форме двойственная к (6.1) задача линейного программирования принимает форму

$$
\langle c, \lambda\rangle \rightarrow \min , \quad \lambda \in \Lambda(A, b):=\left\{\lambda \in \mathbb{R}_{+}^{m} \mid A^{T} \lambda-b=0\right\}
$$

Тесная связь между задачами (6.1) и (6.2) раскрывается в следующей теореме (см. [62]).

Теорема 6.2. Точка $\widehat{U} \in \mathbb{U}(A, c)$ является решением задачи (6.1) тогда и только тогда, когда существует точка $\hat{\Lambda} \in \Lambda(A, b)$ со свойством

$$
\langle c, \widehat{U}\rangle=\langle b, \hat{\Lambda}\rangle
$$

6.2. Задача линейного программирования, двойственная к $(\star)$. Проблема (^) формулируется в форме (6.1) следуюшим образом:

$$
\langle V, U\rangle \rightarrow \max , \quad U \in \mathbb{U}(\mathbb{A}, c)
$$


где $N:=n(n+1)$ и $c=\left(c_{1}, \ldots, c_{N}\right) \in \mathbb{R}^{N}, \mathbb{A}=\left\{a_{i, j}\right\}_{N \times(n+1)}$ определяется компонентами

$$
a_{n i+j, i}=-1, \quad a_{n i+j, j}=1, \quad c_{n i+j, i}=\omega(|j-i|), \quad 0 \leqslant i \neq j \leqslant n .
$$

Все другие компоненты $\mathbb{A}$ и $c$ равны нулю. Тогда задача, двойственная к (6.4), принимает форму

$$
\begin{gathered}
\sum_{0 \leqslant i<j \leqslant n} \omega(|j-i|)\left[\lambda_{n i+j}+\lambda_{n j+i}\right] \rightarrow \min , \\
\sum_{\substack{j=0 \\
j \neq i}}^{n}\left[\lambda_{n j+i}-\lambda_{n i+j}\right]=v_{i}, \quad i=0, \ldots, n ; \quad \lambda_{i} \geqslant 0, \quad i=1, \ldots, N .
\end{gathered}
$$

Таблицы $5.16,5.17$ в [43] иллюстрируют связь между задачами (6.4) и (6.6).

Пусть $\widehat{U}=Z^{*}$ - экстремальный вектор из (6.4) для задачи ( $\star$ ). Согласно теореме 1.2 экстремальный вектор $\hat{\Lambda}=\left(\hat{\lambda}_{0}, \ldots, \hat{\lambda}_{N}\right) \in \Lambda(\mathbb{A}, b)$, или вектор множителей Лагранжа, выражается через $\left\{v_{i, j}\right\}_{0 \leqslant i, j \leqslant n}$ и $\left\{\alpha_{i}:=\operatorname{sign} v_{i}\right\}_{i=0}^{n}$ посредством формул

$$
\hat{\lambda}_{n j+i}:=\frac{1}{2}\left(1+\alpha_{i}\right) v_{i, j}, \quad \hat{\lambda}_{n i+j}:=\frac{1}{2}\left(1-\alpha_{i}\right) v_{i, j}, \quad 0 \leqslant i \leqslant j \leqslant n .
$$

6.3. Классическая "транспортная задача". Пусть $\left\{a_{i}\right\}_{i=1}^{l}:=\left\{v_{r}, r \in\right.$ $\left.\mathbb{Z}[n] \mid v_{r} \geqslant 0\right\}$ и $\left\{b_{j}\right\}_{j=1}^{m}:=\left\{-v_{t}, t \in \mathbb{Z}[n] \mid v_{t}<0\right\}, l+m=n+1,-$ множества модулей всех неотрицательных и отрицательных координат вектора $V$. Для всех $i=1, \ldots, n$ и $j=1, \ldots, m$ положим

$$
\left.\begin{array}{l}
c_{i, j}:=\omega(|r-t|) \\
u_{i, j}:=\hat{\lambda}_{n t+r}
\end{array}\right\}, \text { если } a_{i}=v_{r}, \quad b_{j}=\left|v_{t}\right| \text { для некоторых } r, t \in \mathbb{Z}[n] .
$$

Заметив из (6.7), что $\hat{\lambda}_{n j+i} \neq 0 \Rightarrow \hat{\lambda}_{n i+j}=0$, и, наоборот, в случае выпуклых $\omega$, используя обозначения (6.8) для трансформации (6.6), мы приходим к эквивалентной формулировке задачи (6.6):

$$
\begin{gathered}
\sum_{i=1}^{l} \sum_{j=1}^{m} c_{i, j} \cdot u_{i, j} \rightarrow \min , \quad u_{i, j} \geqslant 0, \quad i=1, \ldots, l, \quad j=1, \ldots, m ; \\
\sum_{j=1}^{m} u_{i, j}=a_{i}, \quad i=1, \ldots, l ; \quad \sum_{i=1}^{l} u_{i, j}=b_{j}, \quad j=1, \ldots, m .
\end{gathered}
$$

Эта проблема, которую можно интерпретировать как задачу о перемещении масс и обобшение "déblais et remblais" задачи Г. Монжа [57], была поставлена и детально исследована Л. В. Канторовичем [13] (см. также [14]), а также Ф. Л. Хитчкоком [48] как "транспортная задача". 
В нашей постановке, nусть $V=\left(v_{0}, \ldots, v_{n}\right) \in \mathbb{R}_{0}^{(n+1)}$ будет данным вектором спроса и предложения и фабрика, производящая (при $\left.v_{i}>0\right)$ или потребляющая $\left(\right.$ если $\left.v_{i}<0\right) \quad v_{i}$ единии, продукиии, расположена в точке $i=0, \ldots, n$. Проблема заключается в нахождении такой схемы поставок продукиии от производителей $\kappa$ потребителям, при которой транспортные затрать минимальны при условии, что стоимость перемещения единицы продукиии из точки $x$ в точку у равна $\omega(|x-y|)$.

Детальное обсуждение дуальности и ссылки на теоремы двойственности для конечномерных и бесконечномерных (см. п. 7.7) “транспортных задач" даны в [52]. История и современный прогресс в теории “транспортной задачи” широко представлены в [56].

6.4. Экономическая интерпретация основных результатов. Теперь результаты предыдущих параграфов допускают простое экономическое толкование. Элемент $v_{i, j}$ разложения вектора $V$ совпадает с количеством продукции, полученной из (при $\left.v_{i}<0\right)$ или отправленной в (при $\left.v_{i}>0\right) j$-ю фабрику $i$-й фабрикой. Импликация $v_{i} \cdot v_{j}>0 \Rightarrow v_{i, j}=v_{j, i}=0$, сформулированная в теореме 1.2 и установленная в лемме 3.2 , означает, что перемешения грузов между двумя потребителями или двумя производителями запрещены в оптимальной схеме перевозок в случае выпуклой функции стоимости $\omega(|x-y|)$.

Дискретная версия леммы Корнейчука (см. $\S 2$ ) требует, чтобы перевозки от каждого производителя $i=1, \ldots, n-1$ осушествлялись наиболее удаленному из еще не загруженных потребителей при условии, что груз уже был отправлен из точек $0,1, \ldots, i-1$. Согласно $(2.11)$ задача $(*)$ для простого вектора $V$ имеет единственное решение тогда и только тогда, когда все фабрики активны, т.е. они производят или потребляют ненулевое количество продукции, и после отправки груза $i$-й фабрики $(i=0, \ldots, n-2)$ ее ближайший получатель для полного удовлетворения своих нужд требует дополнительной поставки из $(i+1)$-й фабрики.

Следующее определение упрощает интерпретацию критерия единственности в обшем случае $V \in \mathbb{R}_{0}^{n+1}$.

ОПРЕДЕЛЕНИЕ 6.3. Пусть $V \in \mathbb{R}_{0}^{n+1}$ и $\mathbb{I} \subset \mathbb{Z}[n]$ - собственное подмножество в $\mathbb{Z}[n]$. Пара $(V, \mathbb{I})$ образовывает замкнутую транспортную систему, если не сушествует экономической связи между фабриками в $x \in \mathbb{I}$ и $x \in \mathbb{Z}[n] \backslash \mathbb{I}$, т.е. ни одна фабрика в $x \in \mathbb{I}$ не снабжается фабрикой из $x \in \mathbb{Z}[n] \backslash \mathbb{I}$ и наоборот. В терминах элементов разложения вектора $V, v_{i, j}=0$ для всех $i \in \mathbb{I}$ и $j \in \mathbb{Z}[n] \backslash \mathbb{I}$.

Понятно, что $\sum_{i \in \mathbb{I}} v_{i}=0$. Согласно предложению 4.1 задача $(*)$ имеет единственное решение тогда и только тогда, когда ни одна пара $\left(V, \mathbb{I}_{i, j}\right)$ с $\mathbb{I}_{i, j}:=$ $\{i, i+1, \ldots, j\}$ не образовывает замкнутую транспортную систему.

В заключение мы дадим интерпретацию результатов пп. 3.2, 3.3. Если координаты вектора $V \in \mathbb{R}_{0}^{n+1}$ имеют четное число перемен знака, то транспортная сис-

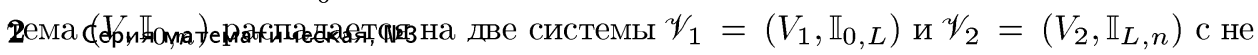


более чем одной фабрикой (в точке $L$ ), имеющей связи с фабриками в обеих транспортных системах.

Если координаты $V$ имеют нечетное число перемен знака, то $\left(V, \mathbb{I}_{0, n}\right)$ распадается на три транспортные системы: $\mathscr{V}_{1}=\left(V_{1}, \mathbb{I}_{K, L}\right), \mathscr{V}_{2}=\left(V_{2}, \mathbb{I}_{L}, M\right)$ и $\mathscr{V}_{3}=$ $\left(V_{3}, \mathbb{I}_{0, K} \cup \mathbb{I}_{M, n}\right)$. При этом единственная возможная связь между $\mathscr{V}_{1}$ и $\mathscr{V}_{2}$ осуществляется в точке $L$. Система $\left(V_{3}, \mathbb{I}_{0, K}\right)$ содержит только производителей (только потребителей), а $\left(V_{3}, \mathbb{I}_{M, n}\right)$ включает в себя лишь потребителей (производителей) соответственно. В соответствии с замечанием 3.10 система $\mathscr{V}_{3}$ может иметь только одну связь с внешним миром: либо с системой $\mathscr{V}_{1}$ в точке $K$, либо с $\mathscr{V}_{2}$ в точке $M$.

\section{§ 7. Приложение к функциональному анализу: экстремали интегральных функционалов}

В этом параграфе мы обсудим непрерывный вариант задачи $(*)$ :

$$
\int_{a}^{b} h(t) \psi(t) d t \rightarrow \sup , \quad h \in H_{a}^{\omega}[a, b]
$$

для ядер $\psi \in \mathbb{L}_{1}[a, b]$ с конечным числом точек перемены знака на $[a, b]$. Как и в дискретном случае, сначала мы изучим строение экстремалей в $(\star)$ для ядер $\psi$ с одной переменой знака и нулевым средним на $[a, b]$. Первообразные $\Psi(x):=$ $\int_{a}^{x} \psi(t) d t$ таких функций называются простыми ядрами. В этом случае и качественное, и количественное решения задачи $(\star)$ в терминах убывающей перестановки ядра $\Psi$ дается леммой Корнейчука. В обшем случае ядер $\psi$ с произвольным конечным числом перемен знака результаты п. 4.3 приводят нас к экстремальному разложению ядра $\Psi$ на такую сумму $\left\{\Phi_{i}(\cdot)=\Phi_{i}(\omega ; \cdot)\right\}_{i=1}^{l}$ простых ядер, которая предохраняет операцию взятия верхней грани в $(\star)$ :

$$
\Psi=\sum_{i=1}^{l} \Phi_{i} ; \quad \sup _{h \in H^{\omega}[a, b]} \int_{a}^{b} h(t) \psi(t) d t=\sum_{i=1}^{l} \sup _{h \in H^{\omega}[a, b]} \int_{a}^{b} h(t) \Phi_{i}^{\prime}(t) d t .
$$

Затем для данных $\xi_{1}, \xi_{2}:\left|\xi_{2}-\xi_{1}\right| \leqslant \omega(b-a)$, мы опишем структуру экстремалей проблемы

$$
\int_{a}^{b} h(t) \psi(t) d t \rightarrow \sup , \quad h \in H^{\omega}[a, b], \quad h(a)=\xi_{1}, \quad h(b)=\xi_{2},
$$

особенно важной в приложениях, связанных с теорией оптимального управления (см. § 10). В заключение мы отметим связь между задачей $(\star)$ в случае $\int_{a}^{b} \psi(x) d x=0$ и непрерывныцм вариантом проблемы Канторовича-Монжа об оптимальном перемещении массы при условии выпуклости функции стоимости. 


\section{1. Лемма Корнейчука и простые функции.}

ОПРЕДЕЛЕНИЕ 7.1. Пусть ядро $\psi \in \mathbb{L}_{1}[a, b]: \int_{a}^{b} \psi(x) d x=0$, таково, что для некоторых точек $a^{\prime}, b^{\prime}: a<a^{\prime} \leqslant b^{\prime}<b$,

$$
\psi(x)<0, \quad x \in\left[a, a^{\prime}\right] ; \quad \psi(x)=0, \quad x \in\left[a^{\prime}, b^{\prime}\right] ; \quad \psi(x)>0, \quad x \in\left[b^{\prime}, b\right]
$$

Тогда для $\xi \in\{ \pm 1\}$ функция $\Psi(x)=\xi \int_{a}^{x} \psi(t) d t$ называется простымм ядром.

Лемма Корнейчука (см. [18], [19]) описьвает экстремальные функции и значение экстремума в $(\star)$ в случае производных $\psi$ простых ядер.

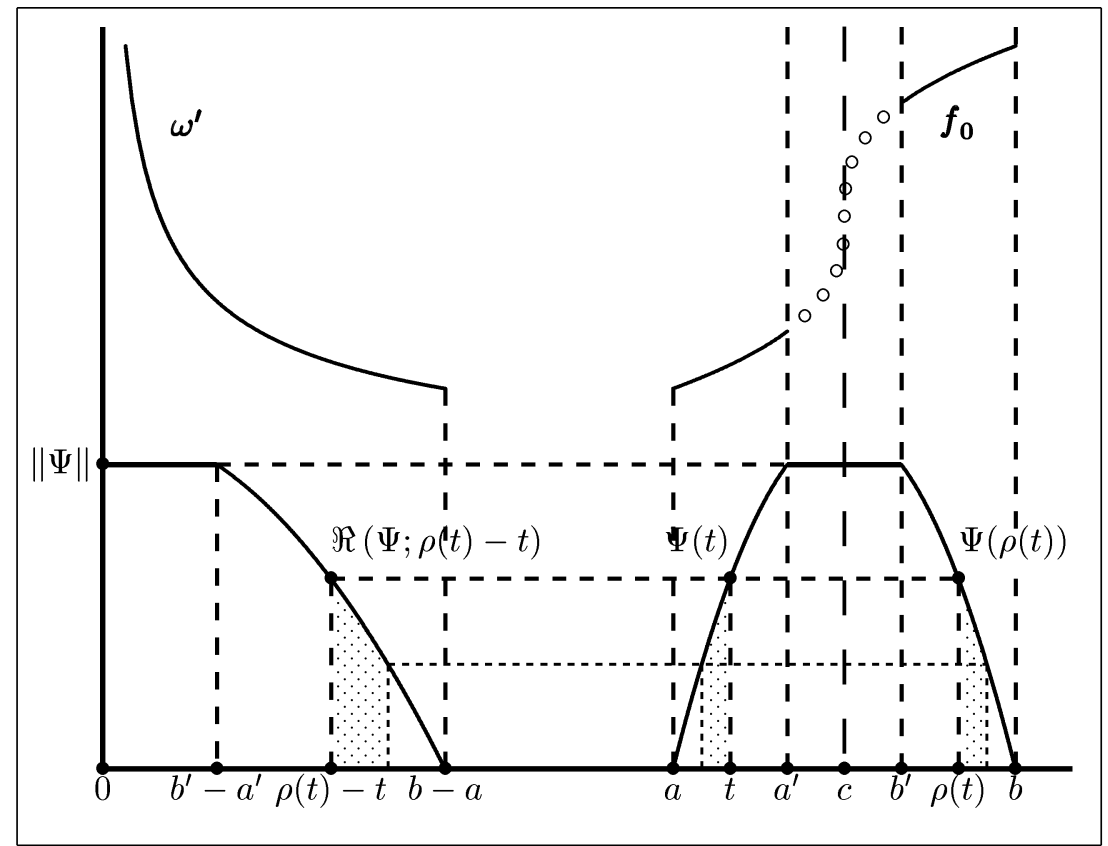

Рис. 7.1. Перестановка $\Re(\Psi ; \cdot)$ простых ядер $\Psi$ и функция $f_{0}$

ЛЕмма 7.2. Пусть производная $\psi$ простого ядра $\Psi(t):=\int_{a}^{t} \psi(y) d y, \quad a \leqslant$ $t \leqslant b$, удовлетворяет неравенствам (7.1) $u \omega$ - выпуклый модуль непрерывности, $c:=\left(a^{\prime}+b^{\prime}\right) / 2$ и $\rho:[a, c] \rightarrow[c, b]$ определена уравнениями

$$
\Psi(t)=\Psi(\rho(t)), \quad t \in\left[a, a^{\prime}\right] ; \quad \rho(t)=a^{\prime}+b^{\prime}-t, \quad t \in\left[a^{\prime}, c\right] .
$$

Тогда

$$
\sup _{f \in H^{\omega}[a, b]} \int_{a}^{b} f(t) \psi(t) d t=\int_{0}^{b-a} \Psi^{*}(t) \omega^{\prime}(t) d t
$$


и верхняя грань в (7.3) достигается на функциях $f_{0}(t)+C$ с производной

$$
\frac{d}{d t} f_{0}(t)= \begin{cases}\omega^{\prime}(\rho(t)-t), & a \leqslant t \leqslant c \\ \omega^{\prime}\left(t-\rho^{-1}(t)\right), & c \leqslant t \leqslant b .\end{cases}
$$

Следуюшее свойство представляет собой непрерывный аналог отношения (2.10) в дискретном случае задачи $(\star)$ для простых векторов: для $t \in[0, c]$

$$
f_{0}(\rho(t))-f_{0}(t)=\int_{c}^{t} \omega^{\prime}(\rho(u)-u)\left[\rho^{\prime}(u)-1\right] d u=\omega(\rho(t)-t) .
$$

\section{2. Обозначения и определения.}

ОБОЗНА чениЕ 7.3. Обозначение $I=\square$ используется для интервалов $I=[\gamma, \gamma]$ с пустой внутренностью.

ОПРеДЕЛЕнИЕ 7.4. Пусть $j \in\{ \pm 1,0\}$ и $n \in \mathbb{N}$. Тогда $\psi \in \mathbb{L}[a, b]$ принадлежuт класcy $\mathscr{M}_{n}^{j}[a, b]$ при $n \geqslant 2$ тогда и только тогда, когда $\operatorname{sign} \int_{a}^{b} \psi(x) d x=j$ и

$$
(-1)^{i} \psi(t)>0, \quad t \in\left(\alpha_{i-1}, \alpha_{i}\right), \quad i=1, \ldots, n,
$$

для некоторых $a=\alpha_{0}<\alpha_{1}<\cdots<\alpha_{n-1}<\alpha_{n}=b$.

ОПРЕДЕЛЕНИЕ 7.5. Для $n \in \mathbb{N}$ множества индексов $\left\{J_{i}(n)\right\}_{i \in \mathbb{N}},\left\{L_{i}\right\}_{i \in \mathbb{N}}$ и пар индексов $\mathscr{P}(n)$ определяются следуюшим образом:

$$
\begin{aligned}
J_{i}(n) & = \begin{cases}\varnothing \text { при } i=n-2, n-1, n, \text { если } n \geqslant 3 \text { и при } n<3 ; \\
\{j=i+1+2 k, k \in \mathbb{N} \mid j \leqslant n\}, & 1 \leqslant i \leqslant n-3 ;\end{cases} \\
L_{i} & = \begin{cases}\varnothing, & i=1,2,3 ; \\
\{l=i-1-2 k, k \in \mathbb{N} \mid l \geqslant 1\}, & 4 \leqslant i \leqslant n ;\end{cases} \\
\mathscr{P}(n) & =\left\{(i, j) \in \mathbb{N}^{2} \mid 1 \leqslant i \leqslant n-3, j \in J_{i}(n)\right\} .
\end{aligned}
$$

7.3. $V_{n}^{j}$-разбиения ограниченных интервалов. Структура экстремалей задачи $(\star)$ характеризуется в терминах специальных разбиений промежутка $[a, b]$.

ОПРеДЕЛЕнИЕ 7.6. Пусть $n \in \mathbb{N}, j \in\{ \pm 1,0\}$. Разбиение $\mathscr{V}=\left\{A_{i}, B_{i}, C_{i}, D_{i}\right\}_{i=1}^{n}$ отрезка $[a, b]$ на интервалы и подынтервалы $\left\{B_{i j}, C_{j i}\right\}_{(i, j) \in \mathscr{P}(n)}$ называется $V_{n}^{j}$-разбиением при условии, что выполняются следуюшие условия (см. рис. 7.2 ):

(A) $C_{i}=\left[\gamma_{4 i-4}, \gamma_{4 i-3}\right], \quad D_{i}=\left[\gamma_{4 i-3}, \gamma_{4 i-2}\right], \quad B_{i}=\left[\gamma_{4 i-2}, \gamma_{4 i-1}\right], \quad A_{i}=$ $\left[\gamma_{4 i-1}, \gamma_{4 i}\right], i=1, \ldots, n$, для таких $\left\{\gamma_{i}\right\}_{i=0}^{4 n}$, что $a=\gamma_{0} \leqslant \gamma_{1} \leqslant \cdots \leqslant \gamma_{n}=b$;

(B) $C_{i}=\odot, i=1,2,3 ; B_{i}=\odot, i=n-2, n-1, n$;

$\left(\mathrm{C}_{1}\right) j=0 \Longrightarrow D_{i}=\odot, i=1, \ldots, n$;

$\left(\mathrm{C}_{2}\right) j=-1 \Longrightarrow D_{2 k}=\odot, k=1, \ldots,[n / 2]$;

$\left(\mathrm{C}_{3}\right) j=+1 \Longrightarrow D_{2 k-1}=\odot, k=1, \ldots,\lceil n / 2\rceil$; 
(D) $B_{i}=\bigcup_{j \in J_{i}(n)} B_{i j}, \quad 1 \leqslant i \leqslant n-3$, где $B_{i j}=\left[\xi_{i}((j-i+1) / 2)\right.$, $\left.\xi_{i}((j-i-1) / 2)\right], j \in J_{i}(n)$, для таких $\left\{\xi_{i}(k)\right\}_{k=1}^{\left|J_{i}(n)\right|}$, что $\gamma_{4 i-2}=\xi_{i}\left(\left|J_{i}(n)\right|\right) \leqslant$ $\cdots \leqslant \xi_{i}(2) \leqslant \xi_{i}(1)=\gamma_{4 i-1}$

(E) $C_{i}=\bigcup_{l \in L_{i}} C_{i l}, 4 \leqslant i \leqslant n$, где $C_{i l}=\left[\varkappa_{i}((i-l-1) / 2), \varkappa_{i}((i-l+1) / 2)\right]$, $l \in L_{i}$, для таких $\left\{\varkappa_{i}(k)\right\}_{k=1}^{\left|L_{i}\right|}$, что $\gamma_{4 i-4}=\varkappa_{i}(1) \leqslant \varkappa_{i}(2) \leqslant \cdots \leqslant \varkappa_{i}\left(\left|L_{i}\right|\right)=\gamma_{4 i-3}$.

ПримеР 7.7. Перечислим $V_{n}^{j}$-разбиения $[a, b]$ на интервалы $\left\{A_{i}, B_{i}, C_{i}, D_{i}\right\}_{i=1}^{n}$ без учета вырожденных отрезков $A_{N},\left\{B_{i}\right\}_{i=N-2}^{N},\left\{C_{i}\right\}_{i=1}^{3}$ :

$$
\begin{aligned}
N= & 2: D_{1} A_{1} D_{2} ; N=3: D_{1} A_{1} D_{2} A_{2} D_{3} N=4: D_{1} B_{1} A_{1} D_{2} A_{2} D_{3} A_{3} C_{4} D_{4} ; \\
N= & 5: D_{1} B_{1} A_{1} D_{2} B_{2} A_{2} D_{3} A_{3} C_{4} D_{4} A_{4} C_{5} D_{5} ; \\
N=6: & D_{1} B_{1} A_{1} D_{2} B_{2} A_{2} D_{3} B_{3} A_{3} C_{4} D_{4} A_{4} C_{5} D_{5} A_{5} C_{6} D_{6} \\
N \geqslant & 7: D_{1} B_{1} A_{1} D_{2} B_{2} A_{2} D_{3} B_{3}, A_{k} C_{k+1} D_{k+1} B_{k+1}, 3 \leqslant k \leqslant N-4 \\
& A_{N-3} C_{N-2} D_{N-2} A_{N-2} C_{N-1} D_{N-1} A_{N-1} C_{N} D_{N} .
\end{aligned}
$$

Рис. 7.2 поясняет порядок атомов в $V_{8}^{0}$-разбиениях.

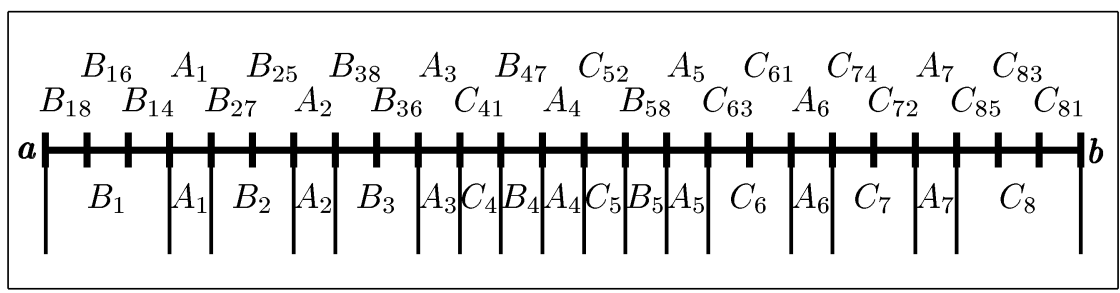

Рис. 7.2. $V_{8}^{0}$-разбиение

7.4. Экстремали интегральных функционалов в $H^{\omega}[a, b]$. Опираясь на понятие $V_{n}^{j}$-разбиений и лемму Корнейчука, в этом пункте мы опишем строение экстремальных функций задачи $(\star)$.

Teорема 7.8. Пусть $\psi \in \mathscr{M}_{n}^{j}[a, b], \quad j \in\{-1,0,+1\}, u\left\{\alpha_{i}\right\}_{i=0}^{n}-$ точки neремены знака $\psi$ из определения 7.4. Тогда существуют решение $x_{\omega, \psi}$ задачи $(\star)$ и $V_{n}^{j}$-разбиение $\mathcal{V}$ интервала $[a, b]$ со следующими свойствами:

(A) $\alpha_{i} \in A_{i}, i=1, \ldots, n-1$;

(B) $\int_{B_{i j} \cup C_{j i}} \psi(t) d t=0,(i, j) \in \mathscr{P}(n)$;

(C) $\int_{A_{i}} \psi(t) d t=0, i=1, \ldots, n-1$;

(D $\left.\mathrm{D}_{1}\right) j=-1 \Longrightarrow x_{\omega, \psi}(t)=-\omega(t-a), t \in D_{2 k-1} \neq \odot, k=1, \ldots,\lceil n / 2\rceil$;

$\left(\mathrm{D}_{2}\right) j=1 \Longrightarrow x_{\omega, \psi}(t)=\omega(t-a), t \in D_{2 k} \neq \odot, k=1, \ldots,[n / 2]$;

(E) для $(i, j) \in \mathscr{P}(n)$ функиия $x_{\omega, \psi}$ әкстремальна в задаче

$$
\begin{array}{ll}
\int_{a}^{b} h(t) \psi_{i j}(t) d t \rightarrow \sup , & h \in H^{\omega}[a, b], \\
\psi_{i j}(t):=\psi(t) \cdot \mathscr{X}\left(B_{i j} \cup C_{j i} ; t\right), & t \in[a, b] ;
\end{array}
$$


(F) при $i=1, \ldots, n-1$ функиия $x_{\omega, \psi}$ әкстремальна в задаче

$$
\begin{array}{cc}
\int_{a}^{b} h(t) \psi_{i}(t) d t \rightarrow \sup , & h \in H^{\omega}[a, b], \\
\psi_{i}(t):=\psi(t) \cdot \mathscr{X}\left(A_{i} ; t\right), & t \in[a, b] .
\end{array}
$$

Соответствуюший вариант теоремы 7.8 для ядер $\psi$ с нулевым средним был впервые установлен в работе автора [2] как приложение теоремы Брауэра.

7.5. Структурные свойства экстремалей. Прежде всего заметим, что все ядра

$\Psi_{i j}(t)=\int_{a}^{t} \psi_{i j}(y) d y, \quad(i, j) \in \mathscr{P}(n), \quad \Psi_{i}(t)=\int_{a}^{t} \psi_{i}(y) d y, \quad i=1, \ldots, n-1$,

просты в смысле определения 7.1. Действительно, из включений $\alpha_{i} \in A_{i}=$ $\left[\gamma_{4 i-1}, \gamma_{4 i}\right], i=1, \ldots, n-1$, и порядка атомов в $V_{n}^{j}$-разбиениях (см. пример 7.7 ) мы заключаем, что

$$
\operatorname{sign} \psi(t)= \begin{cases}(-1)^{i} & \text { на } \begin{cases}B_{i j}, & (i, j) \in \mathscr{P}(n), \\ {\left[\alpha_{i}^{1}, \alpha_{i}\right],} & i=1, \ldots, n-1 ;\end{cases} \\ (-1)^{i+1} & \text { на } \begin{cases}C_{j i}, & (i, j) \in \mathscr{P}(n), \\ {\left[\alpha_{i}, \alpha_{i}^{2}\right],} & i=1, \ldots, n-1,\end{cases} \end{cases}
$$

где $\left[\alpha_{i}^{1}, \alpha_{i}^{2}\right]:=A_{i}, i=1, \ldots, n-1$, и

$$
\operatorname{sign} \psi(t)= \begin{cases}-1 \text { на } D_{2 i-1}, & i=1, \ldots,\lceil n / 2\rceil, \\ 1 \text { на } D_{2 i}, & i=1, \ldots,[n / 2] .\end{cases}
$$

Согласно (7.10) и утверждениям (В) и (С) теоремы 7.8 функции $\left\{\Psi_{i j}\right\}_{(i, j) \in \mathscr{P}(n)}$ и $\left\{\Psi_{i}\right\}_{i=1}^{n-1}$ из (7.9) являются простыми ядрами. Тогда лемма 7.2 Корнейчука приводит нас к следуюшим формулам для производной $\frac{d}{d t} x_{\omega, \psi}(t)$ :

$$
\frac{d}{d t} x_{\omega, \psi}(t)= \begin{cases}(-1)^{i+1} \omega^{\prime}\left(\rho_{i j}(t)-t\right), & t \in B_{i j}, \\ (-1)^{i+1} \omega^{\prime}\left(t-\rho_{i j}^{-1}(t)\right), & t \in C_{j i},\end{cases}
$$

для всех $(i, j) \in \mathscr{P}(n)$, где $\rho_{i j}: B_{i j} \rightarrow C_{j i}$ определяются из уравнений

$$
\Psi_{i j}(t)=\Psi_{i j}\left(\rho_{i j}(t)\right), \quad t \in B_{i j}, \quad \rho_{i j}(t) \in C_{j i},
$$

и

$$
\frac{d}{d t} x_{\omega, \psi}(t)= \begin{cases}(-1)^{i+1} \omega^{\prime}\left(\rho_{i}(t)-t\right), & t \in\left[\alpha_{i}^{1}, \alpha_{i}\right] \\ (-1)^{i+1} \omega^{\prime}\left(t-\rho_{i}^{-1}(t)\right), & t \in\left[\alpha_{i}, \alpha_{i}^{2}\right]\end{cases}
$$




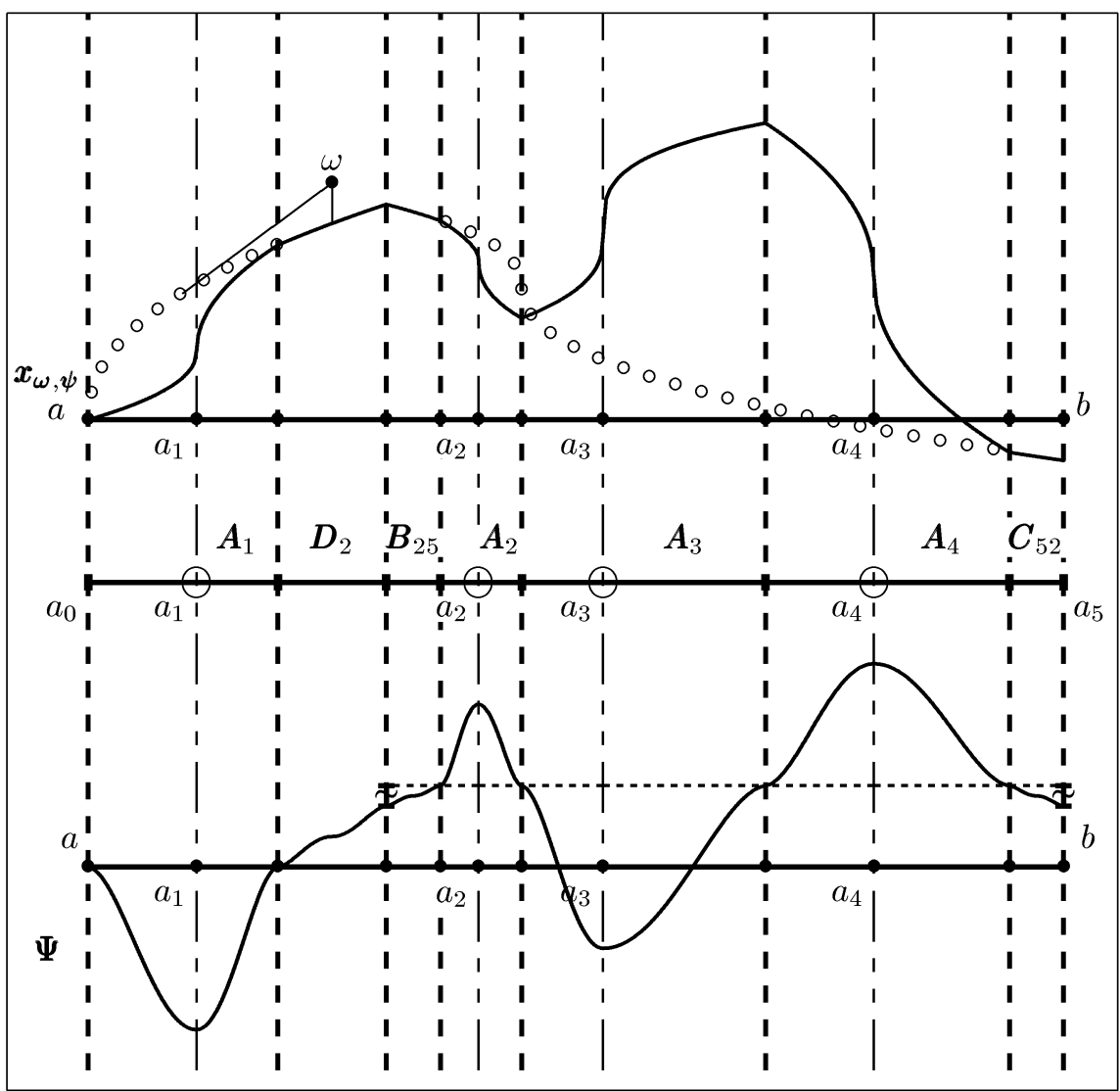

Рис. 7.3. $V_{5}^{+1}$-разбиение и графики $x_{\omega, \psi}$ и $\Psi$ для $\psi \in \mathscr{M}_{5}^{+1}[a, b]$

где $\rho_{i}:\left[\gamma_{4 i-1}, \alpha_{i}\right] \rightarrow\left[\alpha_{i}, \gamma_{4 i}\right]$ определяется из соотношений

$$
\Psi_{i}(t)=\Psi_{i}\left(\rho_{i}(t)\right), \quad t \in\left[\alpha_{i}^{1}, \alpha_{i}\right], \quad \rho_{i}(t) \in\left[\alpha_{i}, \alpha_{i}^{2}\right]
$$

Рис. $7.3,7.4$ иллюстрируют схематические графики экстремальных функций $x_{\omega, \psi}$ для $\psi \in \mathscr{M}_{5}^{l}[a, b], l= \pm 1$, и ядер $\Psi$.

Следствием формул (7.13) и (7.14) является следующая теорема единственности.

ПРЕДЛОЖЕНИЕ 7.9. Если $\psi \in \mathscr{M}_{n}^{j}[a, b]$, то задача $(\star)$ имеет единственное решение.

Из свойства (7.4), примененного к функциям $\left\{\rho_{i, j}\right\}_{(i, j) \in \mathscr{P}(n)}$ из $(7.13)$, следует, ЧTO

$$
\begin{aligned}
\left|x_{\omega, \psi}\left(\rho_{i}(t)\right)-x_{\omega, \psi}(t)\right|=\omega\left(\rho_{i}(t)-t\right), \quad i=1, \ldots, n-1 ; \\
\left|x_{\omega, \psi}\left(\rho_{i, j}(t)\right)-x_{\omega, \psi}(t)\right|=\omega\left(\rho_{i, j}(t)-t\right), \quad t \in B_{i, j}, \quad(i, j) \in \mathscr{P}(n) .
\end{aligned}
$$

Сочетание (7.16) и леммы 3.5 приводят нас к следующему результату. 


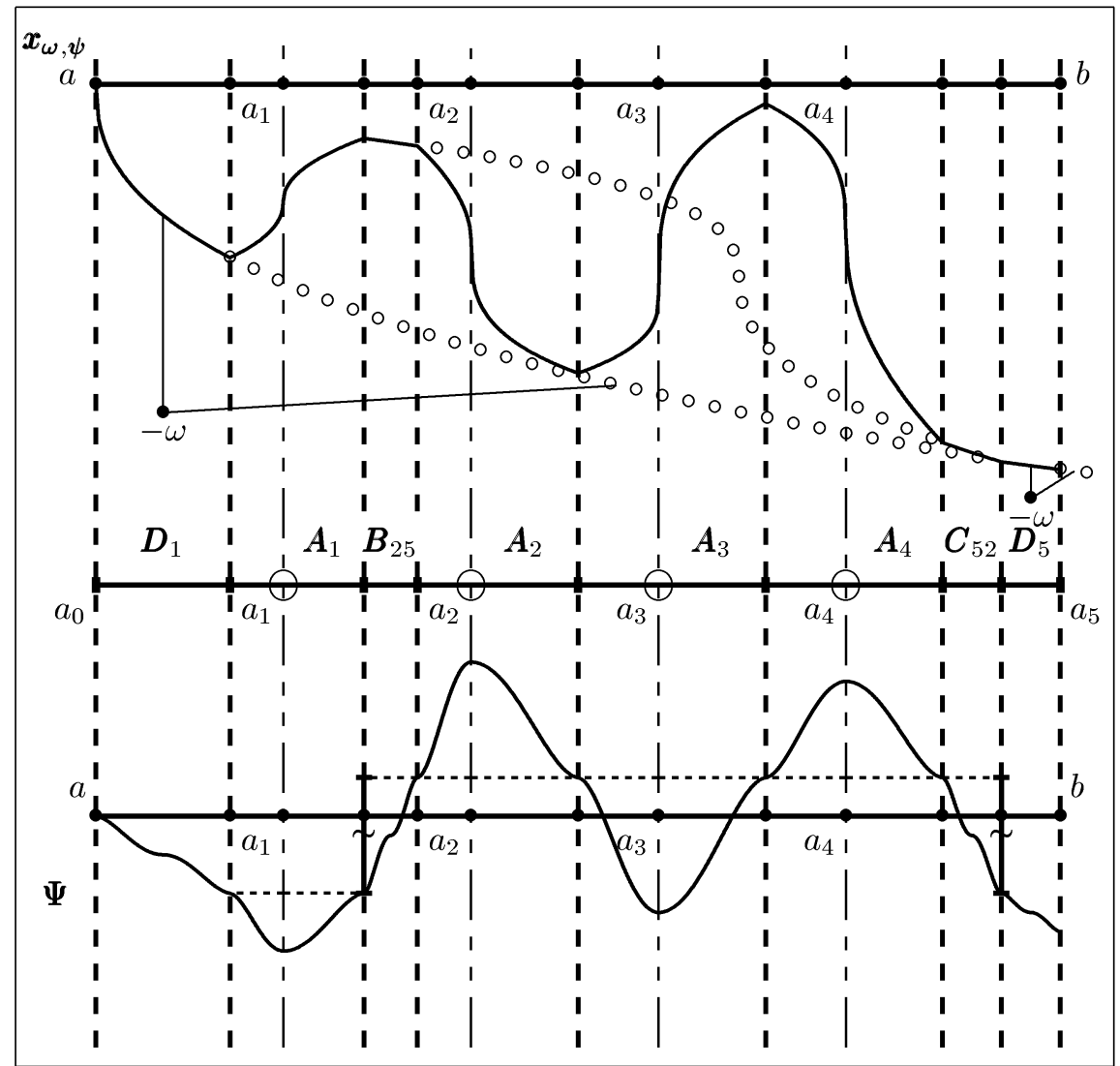

Рис. 7.4. $V_{5}^{-1}$-разбиение и графики $x_{\omega, \psi}$ и $\Psi$ для $\psi \in \mathscr{M}_{5}^{-1}[a, b]$

ПРЕДЛОЖЕНИЕ 7.10. Пусть $B_{i_{1}, j_{1}} \neq \odot, \quad B_{i_{2}, j_{2}} \neq \odot$. Тогда $\left(i_{1}, j_{1}\right) \cap$ $\left(i_{2}, j_{2}\right)=\varnothing$ или $\left(i_{1}, j_{1}\right) \subseteq\left(i_{2}, j_{2}\right)$, или $\left(i_{2}, j_{2}\right) \subseteq\left(i_{1}, j_{1}\right)$.

ОПРЕДЕЛЕНИЕ 7.11. Перестановка $\Re_{\omega}(\Psi ; \cdot)$ определяется формулой

$$
\Re_{\omega}(\Psi ; x):=\sum_{i=1}^{n-1} \Psi_{i}^{*}(x)+\sum_{(i, j) \in \mathscr{P}(n)} \Psi_{i, j}^{*}(x)+|\Psi(x+a)| \sum_{i=0}^{n} \mathscr{X}\left(D_{i} ; x\right),
$$

где простые ядра $\left\{\Psi_{i}\right\}_{i=1}^{n-1}$ и $\left\{\Psi_{i, j}\right\}_{(i, j) \in \mathscr{P}(n)}$ были введены в (7.9).

Тогда формула (7.3), примененная к простым ядрам в (7.9), дает числовое решение задачи $(\star)$.

СЛЕДСТВИЕ 7.12. Если $\omega-$ выпуклый модуль непрерывности, то

$$
\sup _{h \in H_{a}^{\omega}[a, b]} \int_{a}^{b} h(t) \psi(t) d t=\int_{0}^{b-a} \Re_{\omega}(\Psi ; x) \omega^{\prime}(x) d x .
$$


7.6. Специальные варианты задачи $(\star)$. В описании экстремалей задачи

$$
\int_{a}^{b} h(t) \psi(t) d t \rightarrow \sup , \quad h(a)=\xi_{1}, \quad h(b)=\xi_{2},
$$

мы опираемся на теорему 7.8 .

Tеорема 7.13. Если $\left|\xi_{1}-\xi_{2}\right| \leqslant \omega(b-a)$, то существует такое $\tau \in(a, b)$, чmo

$\sup _{\substack{h \in H^{\omega}[a, b] \\ h(a)=\xi_{1}, h(b)=\xi_{2}}} \int_{a}^{b} h(t) \psi(t) d t=\sup _{\substack{h \in H^{\omega}[a, \tau] \\ h(a)=\xi_{1}}} \int_{a}^{\tau} h(t) \psi(t) d t+\sup _{\substack{h \in H^{\omega}[\tau, b] \\ h(b)=\xi_{2}}} \int_{\tau}^{b} h(t) \psi(t) d t$.

Доказательство результата и характеризация точек $\tau$ со свойством, сформулированным в теореме 7.13 , аналогично нашим рассуждениям в $\S 3$.

ЗАмЕчАниЕ 7.14. Пусть $X$ - экстремальная функция задачи (7.19),

$$
\begin{aligned}
& A:=\max \{\alpha \in(a, b]|| X(\alpha)-X(a) \mid=\omega(\alpha-a)\}, \\
& B:=\min \{\beta \in[a, b)|| X(b)-X(\beta) \mid=\omega(b-\beta)\}
\end{aligned}
$$

и $\left\{\tau_{i}\right\}_{i=0}^{k}, A=: \tau_{0}<\tau_{1}<\cdots<\tau_{k-1}<\tau_{k}:=B,-$ максимальная система точек, наделенная свойством

$$
\left|X\left(\tau_{i}\right)-X\left(\tau_{i-1}\right)\right|=\omega\left(\tau_{i}-\tau_{i-1}\right), \quad i=1, \ldots, k
$$

Точки $\left\{\tau_{i}\right\}_{i=0}^{k}$ исчерпьвают множество точек $\tau$ со свойством из теоремы 7.13. Если множество $\left\{\tau_{i}\right\}_{i=1}^{k-1}$ непусто, то

$$
\int_{\tau_{i-1}}^{\tau_{i}} \psi(t) d t=0, \quad i=1, \ldots, k .
$$

Следовательно, интервал $\left[\tau_{i-1}, \tau_{i}\right]$ содержит по меньшей мере одну перемену знака функции $\psi$ для $i=1, \ldots, k-1$. Таким образом, число таких точек $\tau$ не превосходит $l+1$, где $l$ - число точек перемен знака ядра $\psi$.

Теорема 7.13 играет важную роль в задачах быстродействия теории тотального контроля и служит основой для новой формулировки принципа динамического программирования Беллмана (см. § 10 и [9], [10]).

Пусть $a<0<b$. Результаты п. 3.4 и теорема 7.8 дают нам возможность охарактеризовать структуру экстремалей задачи

$$
\int_{a}^{b} h(t) \psi(t) d t \rightarrow \sup , \quad h \in H_{0}^{\omega}[a, b] .
$$


Teорема 7.15. Пусть $\psi \in \mathscr{M}_{n}^{j}[a, b], \quad a<0<b$. Существуют такие точки $\tau_{1} \in[a, 0) u \tau_{2} \in(0, b]$, чmo

$$
\int_{a}^{\tau_{1}} \psi(t) d t+\int_{\tau_{2}}^{b} \psi(t) d t=0
$$

$u$

$$
\begin{aligned}
\sup _{h \in H_{0}^{\omega}[a, b]} & \int_{a}^{b} h(t) \psi(t) d t=\sup _{h \in H_{0}^{\omega}\left[\tau_{1}, 0\right]} \int_{\tau_{1}}^{0} h(t) \psi(t) d t \\
& +\sup _{H_{0}^{\omega}\left[0, \tau_{2}\right]} \int_{0}^{\tau_{2}} h(t) \psi(t) d t+\sup _{h \in H^{\omega}[a, b]} \int_{a}^{b} h(t) \widehat{\psi}(t) d t
\end{aligned}
$$

əде $\widehat{\psi}(t):=\psi(t) \cdot \mathscr{X}\left(\left[a, \tau_{1}\right] \cup\left[\tau_{2}, b\right] ; t\right), \quad t \in[a, b]$.

Теорема 7.15 необходима при решении следуюшего варианта задачи Колмогорова-Ландау о максимизации последней производной $f^{(r)}(\xi)$ функции $f \in W^{r} H^{\omega}(\mathbb{I})$ во внутренней точке интервала $\mathbb{I}$ (см. [33], [61]):

$$
f^{(r)}(\xi) \rightarrow \sup , \quad f \in W^{r} H^{\omega}(\mathbb{I}), \quad\|f\|_{\mathbb{L}_{\infty}(\mathbb{I})} .
$$

\section{§ 8. Приложения к теории приближений: $\omega$-полиномы Чебышева и $\omega$-сплайны}

В данном параграфе мы сначала напомним читателю некоторые задачи в теории соболевских классов, в которых многочлены и сплайны Чебышева естественным образом возникают в качестве экстремальных функций. Взяв за основу характеристическое свойство этих классических функций максимизировать норму промежуточных производных, мы построим аналог чебышевских многочленов в классах $W^{r} H^{\omega}[0,1]$. Наконец, мы затронем некоторые аспекты общей теории неравенств Колмогорова-Ландау в классах $W^{r} H^{\omega}(\mathbb{I})$.

8.1. Классические чебышевские многочлены. Многочлен Чебышева [18] степени $r+1$ с главным коэффициентом $1 /(r+1)$ ! определяется формулой

$$
T_{r}(x):=\frac{2^{-2 r-1}}{(r+1) !} \cos [(r+1) \arccos (2 x-1)], \quad x \in[0,1] .
$$

Согласно (8.1) $L_{r}:=2^{-2 r-1} /(r+1)$ ! совпадает с $C[0,1]$-нормой $T_{r}$, а набор точек $\left\{\vartheta_{i}=(1 / 2)(1+\cos (\pi i /(r+1)))\right\}_{i=0}^{r+1}$ образует множество точек альтернанса функции $T_{r}(x)$ на $[0,1]$ :

$$
T_{r}\left(\vartheta_{i}\right)=(-1)^{r+1+i}\left\|T_{r}\right\|_{\mathbb{C}[0,1]}=(-1)^{r+1+i} L_{r}, \quad i=0, \ldots, r+1 .
$$

Чебьшевские многочлены фигурируют в разнообразных задачах теории приближения, включая проблему нахождения функции наименьшего уклонения 
от нуля среди всех многочленов с одним или двумя ведущими коэффициентами (см. [12], [1]), вычисление $n$-поперечников соболевских классов [28], [26], задачу о точных неравенствах Колмогорова - Ландау на конечном интервале и связанную с ней проблему оптимальных формул численного дифференцирования (см. [64], [50], [61]). Именно последним двум задачам мы и уделим особое внимание в этом параграфе.

Пусть $r, m$ - целые числа, $0<m \leqslant r$. Классическое неравенство Маркова утверждает, что

$$
\left\|p^{(m)}\right\|_{C[0,1]} \leqslant\left\|T^{(m)}\right\|_{\mathbb{C}[0,1]}=\left|T^{(m)}(0)\right|
$$

для всех многочленов $p$ степени $r+1$ с главным коэффициентом $1 /(r+1)$ ! Более того, полином Чебышева $T_{r}$ экстремален в задаче

$$
\left|f^{(m)}(0)\right| \rightarrow \sup , \quad f \in W_{\infty}^{r+1}[0,1], \quad\|f\|_{C[0,1]} \leqslant L_{r}
$$

где соболевский класс определяется следующим образом:

$$
W_{\infty}^{n}[a, b]:=\left\{f \in A C^{n-1}[a, b] \mid\left\|f^{(n)}\right\|_{\mathbb{L}_{\infty}[a, b]} \leqslant 1\right\}
$$

В наших конструкциях мы будем опираться на следуюшее свойство полиномов Чебышева.

ЛЕмма 8.1. Пусть функиия $h \in C^{r}[0,1]$ с абсолютно непрерывной производной $h^{(r)}(\cdot)$ такова, что $h^{(r+1)}(t) \geqslant E$ для $n$. в. $t \in[0,1]$. Тогда

$$
\|h\|_{\mathbb{C}[0,1]} \geqslant E\|T\|_{\mathbb{C}[0,1]}=E L_{r}
$$

ДокАЗАТЕЛЬСТво. Пусть коэффициенты ядра $F(t)=(1 / r !) \sum_{i=0}^{r+1} \alpha_{i}\left(\vartheta_{i}-t\right)_{+}^{r}$ являются решениями системы линейных уравнений

$$
\sum_{i=0}^{r+1} \alpha_{i} \vartheta_{i}^{j}=0, \quad j=0, \ldots, r ; \quad \sum_{i=0}^{r+1}(-1)^{r+1+i} \alpha_{i}=1
$$

где $\left\{\vartheta_{i}\right\}_{i=0}^{r+1}-$ точки альтернанса $T$. Легко проверяется (либо непосредственно, либо используя лемму Ролля), что $(-1)^{r+1+i} \operatorname{sign} \alpha_{i}>0$ для $i=0, \ldots, r+1$ и что $F(t)>0$ для всех $t \in(0,1)$. Тогда приложение формулы Тейлора приводит нас к двум соотношениям

$$
\begin{aligned}
\|T\|_{C[0,1]} & =\sum_{i=0}^{r+1}(-1)^{r+1+i} \alpha_{i} T\left(\vartheta_{i}\right)=\int_{0}^{1} T^{(r+1)} F(t) d t=\|F\|_{\mathbb{L}_{1}[0,1]}, \\
\|h\|_{\mathbb{C}[0,1]} & \geqslant \sum_{i=0}^{r+1}(-1)^{r+1+i} \alpha_{i} h\left(\vartheta_{i}\right)=\int_{0}^{1} h^{(r+1)} F(t) d t \geqslant E\|F\|_{\mathbb{L}_{1}[0,1]},
\end{aligned}
$$

доказывающим (8.5). 
8.2. Чебышевские $\omega$-полиномы. Для данных $r, m \in \mathbb{N}: 0<m \leqslant r$, мы построим аналоги $T_{r, m}$ чебышевского многочлена $T_{r}$ в классе $W^{r} H^{\omega}[0,1]$, наделенные свойством экстремальности в задаче

$$
\left|f^{(m)}(0)\right| \rightarrow \sup , \quad f \in W^{r} H^{\omega}[0,1], \quad\|f\|_{\mathbb{C}[0,1]} \leqslant B
$$

для $B=L_{r, m}:=\left\|T_{r, m}\right\|_{\mathbb{C}[0,1]}$ (cр. с (8.2) и (8.4)).

Подобно своему прототипу в соболевском классе функция $T_{r, m}$ осциллирует между $r+1$ точкой альтернанса на $[0,1]$, и $T_{r, m}^{(r)}$ имеет полный модуль непрерывности: $\omega\left(T_{r, m}^{(r)} ; t\right)=\omega(t), t \in[0,1]$.

Пусть $\tau=\left\{\tau_{i}\right\}_{i=0}^{r+1}$ удовлетворяют $0=\tau_{0}<\tau_{1}<\cdots<\tau_{r+1}=1$. Пусть коэффициенты $\left\{\alpha_{i}\right\}_{i=0}^{r}$ ядер

$$
K_{\tau}(u)=-\frac{1}{(r-1) !} \sum_{i=0}^{r} \alpha_{i}\left(\tau_{i}-u\right)_{+}^{r-1}, \quad F_{\tau}(u)=\frac{1}{r !} \sum_{i=0}^{r} \alpha_{i}\left(\tau_{i}-u\right)_{+}^{r}
$$

являются решениями уравнений

$$
\sum_{i=0}^{r} \alpha_{i} \tau_{i}^{k}=m ! \cdot \delta_{m, k}, \quad k=0, \ldots, r .
$$

Из теоремы Ролля можно вывести, что $(-1)^{i+m} \operatorname{sign} \alpha_{i}>0, i=0, \ldots, r$, и $F_{\tau}^{(k)}(\cdot)$ имеет ровно $k$ простых нулей на $\left(0, \tau_{r}\right)$ при $k=0, \ldots, r-m$ и $k-1$ простых нулей при $k=r-m+1, \ldots, r-1$. В частности, $F_{\tau}$ - простое ядро на $\left(0, \tau_{r}\right)$ в смысле определения 7.1 при $0<m<r$ и $K=F^{\prime}$ сохраняет постоянный знак на $\left(0, \tau_{r}\right)$, если $m=r$. Следствием формулы Тейлора

$$
f(\tau)=\sum_{k=0}^{r-1} \frac{f^{(k)}(0)}{k !} \tau^{k}+\frac{1}{(r-1) !} \int_{0}^{1} f^{(r)}(u)(\tau-u)_{+}^{r-1} d u, \quad 0 \leqslant \tau \leqslant 1,
$$

является формула численного дифференщирования

$$
f^{(m)}(0)=\sum_{i=0}^{r} \alpha_{i} f\left(\tau_{i}\right)+\int_{0}^{b}\left[f^{(r)}(u)-f^{(r)}(0)\right] K_{\tau}(u) d u .
$$

В построении чебышевских $\omega$-полиномов мы опираемся на теорему Брауэра о неподвижной точке [63].

TeOpema 8.2. Пусть

$$
\Sigma^{n}=\left\{s=\left(s_{1}, \ldots, s_{n+1}\right) \in \mathbb{R}_{+}^{n+1} \mid \sum_{i=1}^{n+1} s_{i}=1\right\} .
$$

Если $\sigma$ - непрерьвное отображение из $\Sigma^{n}$ в $\Sigma^{n}$, то существует такая точка $\xi^{*} \in \Sigma^{n}$, что $\sigma\left(\xi^{*}\right)=s^{*}$. 
TЕорема 8.3. Существуют такой набор точек $\tau=\left\{\tau_{i}=\tau_{i}(r, m, \omega)\right\}_{i=0}^{r+1}$, $0=\tau_{0}<\cdots<\tau_{r+1}=1$, и такая функиия $\mathscr{Z} \in H^{\omega}[0,1]$, что:

i) $\sup _{h \in H_{0}^{\omega}\left[0, \tau_{r}\right]} \int_{0}^{\tau_{r}} h(t) K_{\tau}(t) d t=\int_{0}^{\tau_{r}}\left[\mathscr{Z}^{(r)}(t)-\mathscr{Z}^{(r)}(0)\right] K_{\tau}(t) d t$;

ii) $\mathscr{Z}\left(\tau_{i}\right)=(-1)^{i+m}\|\mathscr{Z}\|_{C[0,1]}, \quad i=0, \ldots, r+1$.

Во избежание несущественных технических выкладок мы проведем доказательство результата для всех модулей непрерывности $\omega$ со свойством $\omega^{\prime}(x) \geqslant \omega_{0}$ для некоторого $\omega_{0}>0$ и п.в. $x \in[0,1]$.

ДоКАЗАТЕЛЬСТВО. Пусть $\Sigma^{r+1}-(r+1)$-мерньй симплекс из (8.11). Для фиксированного $\varepsilon>0$ и данного $s=\left(s_{1}, \ldots, s_{r+2}\right) \in \Sigma^{r+1}$ построим множества точек $\left\{t_{j}(s)\right\}_{j=0}^{r+1}$ и $\left\{\tau_{j}=\tau_{j}(s)\right\}_{j=0}^{r+1}$ по правилу

$$
\begin{aligned}
t_{0}(s):=0, & t_{j}(s):=\sum_{i=1}^{j}\left|s_{i}\right|, & j=1, \ldots, r+1 \\
\tau_{0}(s):=0, & \tau_{j}(s):=\frac{t_{j}(s)+\varepsilon j}{1+\varepsilon r}, & j=1, \ldots, r .
\end{aligned}
$$

По этому определению следуюшие неравенства справедливы для $s \in \Sigma^{r+1}$ :

$$
\tau_{i}(s)-\tau_{i-1}(s) \geqslant \frac{\varepsilon}{1+\varepsilon r}, \quad\left|t_{i}(s)-\tau_{i}(s)\right| \leqslant 2 r \varepsilon, \quad i=1, \ldots, r+1 .
$$

Коэффициенты $\left\{\alpha_{i}(s)\right\}_{i=0}^{r}$ ядра $K_{s}(t)=-(1 /(r-1) !) \sum_{i=0}^{r} \alpha_{i}(s)\left(\tau_{i}(s)-t\right)_{+}^{r-1}$ удовлетворяют системе линейных уравнений

$$
\sum_{i=0}^{r} \alpha_{i}\left(\tau_{i}(s)\right)^{k}=m ! \delta_{m, k}, \quad k=0, \ldots, r
$$

Через $g_{s}$ на $\left[0, \tau_{r}(s)\right]$ обозначим экстремаль задачи

$$
\int_{0}^{\tau_{r}(s)} h(t) K_{s}(t) d t \rightarrow \sup , \quad h \in H_{0}^{\omega}\left[0, \tau_{r}(s)\right]
$$

Если $0<m<r$, то $K_{s}$ - простое ядро и формулы для $\left.g_{s}\right|_{\left[0, \tau_{r}(s)\right]}$ даны в лемме 7.2 Корнейчука. Если $m=r$, то $g_{s}(t)=-\omega(t)$, поскольку $\operatorname{sign} K_{s}=-1$ на $\left(0, \tau_{r}(s)\right)$.

Продолжим $g_{s}$ на интервал $\left[\tau_{r}(s), 1\right]$ по непрерьвности формулой $g_{s}(t):=$ $(-1)^{m+r+1} \omega(t), t \in\left[\tau_{r}(s), 1\right]$, и через $G_{s}$ обозначим следующий $(r-1)$-й интеграл от $g_{s}$ :

$$
G_{s}(x)=\frac{1}{(r-2) !} \int_{0}^{1}(x-t)_{+}^{r-2} g_{s}(t) d t, \quad 0 \leqslant x \leqslant 1 .
$$


Пусть $p_{s}(t)$ будет многочленом степени $r-1$, интерполируюшим $G_{s}(t)$ в точ$\operatorname{kax}\left\{t_{i}(s)\right\}_{i=1}^{r}$ :

$$
p_{s}\left(t_{i}(s)\right)=G_{s}\left(t_{i}(s)\right), \quad i=1, \ldots, r .
$$

При этом в случае совпадения некоторых из точек $\left\{t_{i}(s)\right\}_{i=1}^{r}$ интерполируются последующие производные. Тогда функция

$$
H_{s}(x):=G_{s}(x)-p_{s}(x), \quad 0 \leqslant x \leqslant 1,
$$

равна нулю в точках $\left\{t_{i}(s)\right\}_{i=1}^{r}$ :

$$
H_{s}\left(t_{i}(s)\right)=0, \quad i=1, \ldots, r .
$$

Определим наборы точек $\left\{\xi_{i}(s)\right\}_{i=0}^{r+1}, 0=\xi_{0}(s)<\xi_{1}(s)<\cdots<\xi_{r}(s)<$ $\xi_{r+1}(s)=1$, соотношениями

$$
\left\|H_{s}\right\|_{\mathbb{L}_{1}\left[\xi_{i-1}(s), \xi_{i}(s)\right]}=\frac{1}{r+1}\left\|H_{s}\right\|_{\mathbb{L}_{1}[0,1]} .
$$

По теореме Брауэра существует такая $s^{*}=s^{*}(\varepsilon) \in \Sigma^{r+1}$, что множества $\left\{t_{i}\left(s^{*}\right)\right\}_{i=0}^{r+1}$ и $\left\{\xi_{i}\left(s^{*}\right)\right\}$ совпадают:

$$
\xi_{i}\left(s^{*}\right)=t_{i}\left(s^{*}\right), \quad i=0, \ldots, r+1 .
$$

Из (8.21) и (8.22) вытекает, что $0=t_{0}\left(s^{*}\right)<t_{1}\left(s^{*}\right)<\cdots<t_{r+1}\left(s^{*}\right)=1$. Поскольку $H_{s}^{(r)}(t)=g_{s}^{\prime}(t), t \in[0,1]$, и sign $g_{s}^{\prime}=(-1)^{r+m+1}$, теорема Ролля приводит нас к заключению, что $H_{s}$ может иметь не более $r$ нулей на $[0,1]$. Следовательно, согласно (8.20) простые нули $\left\{t_{i}\left(s^{*}\right)\right\}_{i=1}^{r}$ исчерпывают множество нулей функции $H_{s^{*}}$ :

$$
(-1)^{i+m} H_{s^{*}}(t)>0, \quad t \in\left(t_{i-1}\left(s^{*}\right), t_{i}\left(s^{*}\right)\right), \quad i=1, \ldots, r+1 .
$$

Тогда условия (8.21) могут быть переписаны следующим образом:

$$
\int_{t_{i-1}\left(s^{*}\right)}^{t_{i}\left(s^{*}\right)} H_{s^{*}}(t) d t=(-1)^{i+m} \frac{1}{r+1}\left\|H_{s^{*}}\right\|_{\mathbb{L}_{1}[0,1]}, \quad i=1, \ldots, r+1 .
$$

Следовательно, функция

$$
T_{\varepsilon}(x):=\int_{0}^{x} H_{s^{*}(\varepsilon)}(t) d t+(-1)^{m} \frac{1}{2(r+1)}\left\|H_{s^{*}(\varepsilon)}\right\|_{\mathbb{L}_{1}[0,1]}
$$

имеет альтернанс в точках $\left\{t_{i}\left(s^{*}\right)\right\}_{i=0}^{r+1}$ :

$$
T_{\varepsilon}\left(t_{i}\left(s^{*}\right)\right)=(-1)^{i}\left\|T_{\varepsilon}\right\|_{C[0,1]}, \quad i=0, \ldots, r+1 .
$$

По формулам леммы 7.2

$$
(-1)^{r+m+1} T_{\varepsilon}^{(r+1)}(x)=(-1)^{r+m+1} g_{s^{*}(\varepsilon)}(x)=\omega^{\prime}\left(e_{x}\right) \geqslant \omega_{0}
$$

для некоторого $e_{x} \in(0,1)$. Согласно лемме $8.1\left\|T_{\varepsilon}\right\|_{C[0,1]} \geqslant C_{r} \omega_{0}$, следовательно,

$$
t_{i}\left(s^{*}(\varepsilon)\right)-t_{i-1}^{*}\left(s^{*}(\varepsilon)\right) \geqslant \delta, \quad i=1, \ldots, r+1,
$$

для некоторого $\delta>0$, не зависяшего от $\varepsilon$. Переходя к пределу в $C^{r}[0,1]$ при $\varepsilon \rightarrow 0$, мы получаем функцию $T:=\lim _{\varepsilon \rightarrow 0} T_{\varepsilon}$ с требуемыми свойствами (8.12). 


\section{3. Дальнейшие результаты в теории колмогоровских неравенств.} Начальные результаты в теории экстремальных мультипликативных неравенств для промежуточных производных в классах Соболева $W_{\infty}^{n}(\mathbb{I})$ принадлежат Е. Ландау $[51]\left(n=2, \mathbb{I}=\mathbb{R}_{+}\right)$и Ж. Адамару [45] $(n=2, \mathbb{I}=\mathbb{R})$. Описания экстремальных функций задачи

$$
f^{(m)}(0) \rightarrow \sup , \quad f \in W^{r} H^{\omega}(\mathbb{I}), \quad\|f\|_{\mathbb{L}_{\infty}(\mathbb{I})} \leqslant B, \quad 0<m \leqslant r,
$$

для $B>0$ в линейном случае $\omega(t)=t$ были получены А. Н. Колмогоровым [16], [17] $($ в случае $\mathbb{I}=\mathbb{R})$, А. С. Кавареттой и И. Дж. Шенбергом $[40]\left(\mathbb{I}=\mathbb{R}_{+}\right)$и С. Карлиным [50]. Общий случай нелинейных модулей непрерывности $\omega$ во всех трех случаях разобран в работах автора $[3],[31]\left(\mathbb{I}=\mathbb{R}_{+} \vee \mathbb{R}\right)$ и $[32],[33](\mathbb{I}=[0,1])$.

\section{§ 9. Неравенства для перестановок}

Этот параграф, составленный по материалам работы автора [4], проясняет отношения между тремя видами перестановок ядра $\Psi$ : стандартными убьваюшими перестановками $\Psi^{*}(\cdot), \Sigma$-перестановками $\Sigma(\Psi ; \cdot)$ Корнейчука (см. ниже определение 9.2$)$ и $\omega$-перестановками $\Re_{\omega}(\Psi ; \cdot)$, введенными в определении 7.11 . В частности, мы перечислим ряд основных неравенств для сумм, произведений и производных этих трех видов перестановок.

\section{1. Свойства стандартных убывающих перестановок.}

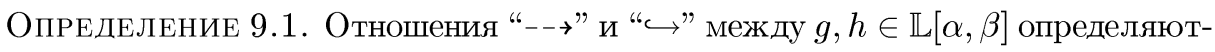
ся следуюшим образом:

$$
\begin{array}{ll}
g \rightarrow h \Longleftrightarrow \int_{\alpha}^{x} g(t) d t \leqslant \int_{\alpha}^{x} h(t) d t & \forall x \in[\alpha, \beta], \\
g \hookrightarrow h \Longleftrightarrow \int_{x}^{\beta} g(t) d t \leqslant \int_{x}^{\beta} h(t) d t & \forall x \in[\alpha, \beta] .
\end{array}
$$

Следуюшие хорошо известные свойства стандартных перестановок играют важную роль в теории интерполящии операторов и пространств, инвариантных относительно перестановок (см. [23], [38] и библиографию в этих книгах).

ПРЕДЛОЖЕНИЕ 9.2. Пусть $x$ и $у$-любье две измеримье функиии на $[a, b]$. Тогда:

i) $\|x\|_{\mathbb{L}_{p}[a, b]}=\left\|x^{*}\right\|_{\mathbb{L}_{p}[0, b-a]} \quad$ для 8 cex $p \geqslant 1$;

ii) $\left(|x|^{p}\right)^{*}(t)=\left(x^{*}\right)^{p}(t), \quad t \in[0, b-a]$;

iii) $\int_{a}^{b} x(t) y(t) d t \leqslant \int_{0}^{b-a} x^{*}(t) y^{*}(t) d t$;

iv) $(x+y)^{*} \rightarrow x^{*}+y^{*}, \quad x^{*}+y^{*} \hookrightarrow(x+y)^{*}$;

v) $x-\rightarrow y \Longrightarrow\left\|x^{*}\right\|_{\mathbb{L}_{p}[0, b-a]} \leqslant\left\|y^{*}\right\|_{\mathbb{L}_{p}[0, b-a]}, \quad p \geqslant 1$. 
В этом параграфе мы зафиксируем ядро $\psi \in \mathscr{M}_{n}^{0}[a, b], n \in \mathbb{N}$ (см. определение 7.4). Как обычно, положим $\Psi(x):=\int_{a}^{x} \psi(t) d t, a \leqslant x \leqslant b$. С этими ядрами мы свяжем точки $\left\{\alpha_{i}\right\}_{i=1}^{n-1}$ перемены знака $\psi$ и нули $\left\{b_{i}\right\}_{i=0}^{r}$ ядра $\Psi$, упорядоченные по возрастанию: $a=: \alpha_{0}<\alpha_{1}<\cdots<\alpha_{n-1}<\alpha_{n}:=b, a=b_{0}<b_{1}<\cdots<b_{r}=b$.

9.2. $\Sigma$-перестановки Корнейчука. $\Sigma$-разбиение Корнейчука представляет собой естественный метод разложения $\Psi$ на сумму простых ядер (см. [20], [21]).

ОПРЕДЕЛЕНИЕ 9.3. Пусть $\rho(\Psi):=\left(\Psi\left(\alpha_{0}\right), \Psi\left(\alpha_{1}\right), \ldots, \Psi\left(\alpha_{n-1}\right)\right)$ - вектор локальных экстремумов $\Psi$, и через $\pi(\Psi)=\left(\pi_{0}, \ldots, \pi_{n-1}\right) \in \mathbb{R}^{n}$ обозначим возрастающую перестановку $\rho(\Psi)$ (понятно, что $\pi_{0}=\min _{a \leqslant t \leqslant b} \Psi(t), \pi_{n-1}=$ $\left.\max _{a \leqslant t \leqslant b} \Psi(t)\right)$. Для $t \in[a, b]$ пусть (см. рис.9.1)

$$
\Psi_{k}(t)= \begin{cases}\min \left\{\max \left\{\Psi(t), \pi_{k-1}\right\}, \pi_{k}\right\}-\pi_{k-1}, & \pi_{k}>0 \\ \min \left\{\max \left\{\Psi(t), \pi_{k-1}\right\}, \pi_{k}\right\}-\pi_{k}, & \pi_{k} \leqslant 0\end{cases}
$$

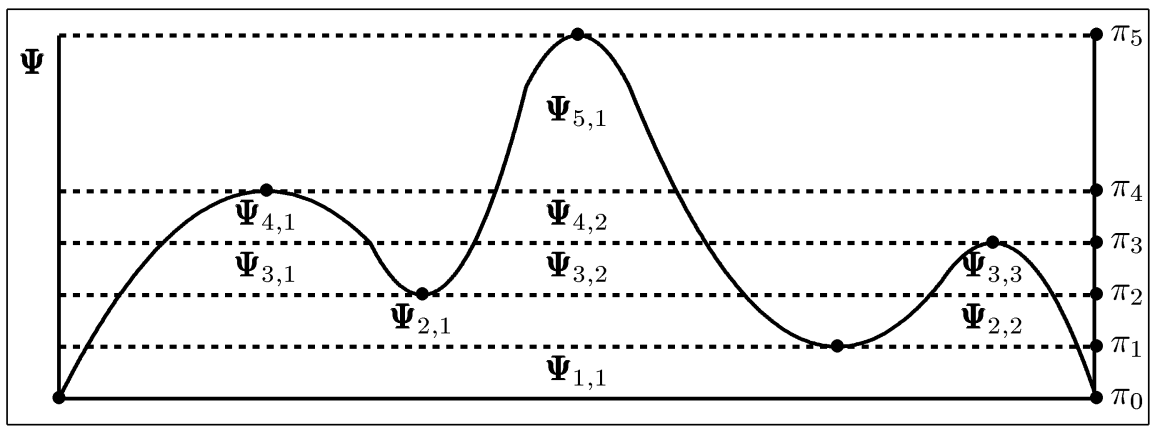

Рис. 9.1. इ-разложение Корнейчука

Тогда $\Psi(\cdot)=\sum_{k=0}^{n-1} \Psi_{n}(\cdot)$ и каждая из функций $\Psi_{k}(t)$ является суммой простых ядер $\left\{\Psi_{k, i}(\cdot)\right\}_{i=1}^{l_{k}}$ с неперекрывающимися носителями. $\Sigma$-разложсение и $\Sigma$-nерестановка $\Sigma(\Psi ; \cdot)$ ядра $\Psi$ вводятся посредством формул

$$
\Psi(t)=\sum_{k=1}^{n-1} \sum_{i=1}^{l_{k}} \Psi_{k, i}(t), \quad t \in[a, b] ; \quad \Sigma(\Psi ; t)=\sum_{k=1}^{n-1} \sum_{i=1}^{l_{k}} \Psi_{k, i}^{*}(t), \quad t \in[0, b-a] .
$$

Отметим некоторые тривиальные свойства $\Sigma$-перестановок:
i) $\|\Sigma(\Psi ; \cdot)\|_{\mathbb{L}[0, b-a]}=\|\Psi\|_{\mathbb{L}[a, b]}$,
ii) $\Sigma(\Psi ; 0)=\frac{1}{2} \bigvee_{a}^{b} \Psi=\frac{1}{2}\|\psi\|_{\mathbb{L}[a, b]}$,
iii) $\Sigma(\lambda \Psi ; \cdot)=|\lambda| \Sigma(\Psi ; \cdot)$,
iv) $\Sigma\left(|\Psi|^{p} ; x\right) \leqslant(\Sigma(\Psi ; x))^{p}$

для всех $x \in[0, b-a]$. 
ПРеДЛОЖЕНИЕ 9.4. Равенство в (9.3), iv) справедливо для всех $x \in$ $[0, b-a]$ тогда и только тогда, когда или $p=1$, или $\Psi$ имеет п нулей $\left\{b_{i}\right\}_{i=1}^{r}$, чередующихся с точками $\left\{\alpha_{i}\right\}_{i=1}^{n-1}$ перемены знака производной $\Psi^{\prime}$ :

$$
b_{i} \leqslant \alpha_{i+1} \leqslant b_{i+1}, \quad i=0, \ldots, n-1 .
$$

9.3. Отношения между перестановками. В этом пункте мы коснемся некоторых аналогов свойств (9.1) для $\Sigma$ - и $\omega$-перестановок. Аббревиатура в.м.н. обозначает вьпуклый модуль непрерьвности.

ОПРЕДЕЛЕНИЕ 9.5. Функции $f, g \in C[c, d]$ связаны отношением $f \diamond g$, если или сушествуют такие точки $x, y \in[c, d]$, что $f(x)>g(x)$ и $f(y)<g(y)$, или $f(x)=$ $g(x)$ для всех $x \in[c, d]$.

Первый результат показьвает, что три перестановки не мажсорируют друг друга.

ПРЕДЛОЖЕНИЕ 9.6. Пусть $\omega$ и $\mu$-любые два в.м.н. Тогда

$$
\begin{aligned}
& \Re_{\omega}(\Psi ; \cdot) \diamond \Re_{\mu}(\Psi ; \cdot), \quad \Re_{\omega}(\Psi ; \cdot) \diamond \Sigma(\Psi ; \cdot), \quad \Re_{\omega}(\Psi ; \cdot) \diamond \Psi^{*}(\cdot), \\
& \Psi^{*}(\cdot) \diamond \Sigma(\Psi ; \cdot), \quad I\left(\Re_{\omega}(\Psi ; \cdot) ; \cdot\right) \diamond I(\Sigma(\Psi ; \cdot) ; \cdot),
\end{aligned}
$$

əде $I(g(\cdot) ; x):=\int_{0}^{x} g(t) d t, 0 \leqslant x \leqslant b-a$.

Следуюшие два результата отвечают на вопрос о совпадении разных видов перестановок.

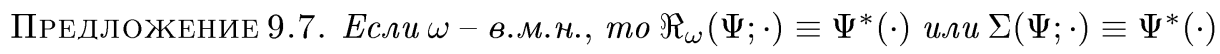
тогда и только тогда, когда $\Psi$ - простое ядро.

ПРЕДЛОЖЕНИЕ 9.8. Пусть $\omega-$ в.м.н. Тогда $\Sigma(\Psi ; \cdot) \equiv \Re_{\omega}(\Psi ; \cdot)$, если нули $\left\{b_{i}\right\}_{i=0}^{n-1}$ ядра $\Psi$ удовлетворяют (9.4) и

$\sum_{l=i}^{j-1}(-1)^{i+l} \omega\left(b_{l+1}-b_{l}\right) \leqslant \omega\left(b_{j}-b_{i}\right), \quad 1 \leqslant i<j \leqslant n: j-i=2 k+1, \quad k \in \mathbb{N}$.

ЗАмЕчАниЕ 9.9 . В случае выполнения неравенств (9.6) экстремальное $V_{n}^{0}$-разбиение задачи $(\star)$ (см. теорему 7.8$)$ содержит только невырожденные $A$-атомы:

$$
A_{i}=\left[b_{i-1}, b_{i}\right], \quad i=1, \ldots, n-1, \quad B_{i}=C_{i}=\left\{b_{i-1}\right\}, \quad i=1, \ldots, n
$$

9.4. Неравенства для перестановок. Неравенства между $\mathbb{L}_{1}(d \omega)$-нормами перестановок устанавливаются в следуюшем результате. 
ПРЕДЛОЖЕНИЕ 9.10. Если $\Psi^{\prime} \in \mathscr{M}_{n}^{0}[a, b], m o:$
i) $\int_{0}^{b-a} \Re_{\omega}(\Psi ; t) \omega^{\prime}(t) d t \leqslant \int_{0}^{b-a} \Re_{\mu}(\Psi ; t) \omega^{\prime}(t) d t \quad$ для любых в.м.н. $\omega, \mu$,
ii) $\int_{0}^{b-a} \Re_{\pi}(\Psi ; t) \sigma^{\prime}(t) d t \leqslant \int_{0}^{b-a} \Re_{\sigma}(\Psi ; t) \pi^{\prime}(t) d t \quad$ для в.м.н. $\sigma, \pi: \sigma \leqslant \pi$,
iii) $\int_{0}^{b-a} \Psi^{*}(t) \omega^{\prime}(t) d t \leqslant \int_{0}^{b-a} \Re_{\omega}(\Psi ; t) \omega^{\prime}(t) d t \leqslant \int_{0}^{b-a} \Sigma(\Psi ; t) \omega^{\prime}(t) d t$

для в.M.н. $\omega$.

Как и в доказательственеравенства (9.1), v) из [41], введенные в определении 9.1

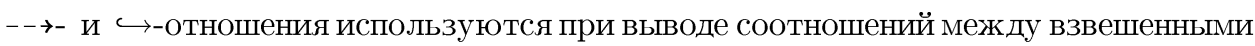
нормами перестановок и версии неравенства (9.1), iii).

ПРЕДЛОЖЕНИЕ 9.11. Пусть $\omega-$ в.м.н. Справедливы следующие отношения:
i) $\quad \Psi^{*} \rightarrow \Sigma(\Psi ; \cdot)$;
ii) $\quad \Psi^{*} \rightarrow \Re_{\omega}(\Psi ; \cdot)$;
iii) $\Sigma(\Psi ; \cdot) \hookrightarrow \Psi^{*}$;
iv) $\Sigma(\Psi ; \cdot) \hookrightarrow \Re_{\omega}(\Psi ; \cdot)$.

СлЕДСТВИЕ 9.12. Пусть $\omega-$ в.м.н.

1. Если $d(\cdot)$ - неотрицательная невозрастающая функиия на отрезке $[0, b-a]$, то $\Psi^{*}(\cdot) d(\cdot) \rightarrow \Sigma(\Psi ; \cdot) d(\cdot)$ u $\Psi^{*}(\cdot) d(\cdot) \rightarrow \Re_{\omega}(\Psi ; \cdot) d(\cdot)$. В частности

$$
\begin{aligned}
& \int_{0}^{b-a} \Psi^{*}(t) d(t) d t \leqslant \int_{0}^{b-a} \Sigma(\Psi ; t) d(t) d t \\
& \int_{0}^{b-a} \Psi^{*}(t) d(t) d t \leqslant \int_{0}^{b-a} \Re_{\omega}(\Psi ; t) d(t) d t .
\end{aligned}
$$

2. Если $c(\cdot)$ - неотрицательная неубывающая функиия на отрезке $[0, b-a]$, то $\Sigma(\Psi ; \cdot) b(\cdot) \hookrightarrow \Psi^{*}(\cdot) b(\cdot)$ и $\Sigma(\Psi ; \cdot) b(\cdot) \hookrightarrow \Re_{\omega}(\Psi ; \cdot) b(\cdot)$. В частности,

$$
\begin{aligned}
& \int_{0}^{b-a} \Sigma(\Psi ; t) c(t) d t \leqslant \int_{0}^{b-a} \Re_{\omega}(\Psi ; t) c(t) d t \\
& \int_{0}^{b-a} \Sigma(\Psi ; t) c(t) d t \leqslant \int_{0}^{b-a} \Psi^{*}(t) c(t) d t .
\end{aligned}
$$

Неравенство (9.7) выполняется для производных $\omega^{\prime}(\cdot)=$ в(·) выпуклых модулей непрерывности $\omega$. Следуюший результат является обобщением неравенства (9.1), iii) Харди-Литтлвуда (см. [46]). 


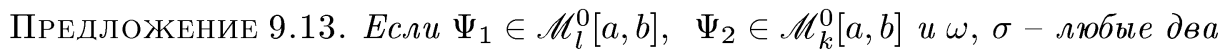
в.M.H., mo

$$
\begin{gathered}
\int_{a}^{b} \Psi_{1}(t) \Psi_{2}(t) d t \leqslant \int_{0}^{b-a} \Re_{\omega}\left(\Psi_{1} ; t\right) \Re_{\sigma}\left(\Psi_{2} ; t\right) d t, \\
\int_{a}^{b} \Psi_{1}(t) \Psi_{2}(t) d t \leqslant \int_{0}^{b-a} \Sigma\left(\Psi_{1} ; t\right) \Re_{\sigma}\left(\Psi_{2} ; t\right) d t, \\
\int_{a}^{b} \Psi_{1}(t) \Psi_{2}(t) d t \leqslant \int_{0}^{b-a} \Sigma\left(\Psi_{1} ; t\right) \Sigma\left(\Psi_{2} ; t\right) d t .
\end{gathered}
$$

В следуюшем результате утверждается, что $\mathbb{L}_{p}$-нормы перестановок $\Sigma(\Psi ; \cdot)$ и $\Re_{\omega}(\Psi ; \cdot)$ мажорируют $\|\Psi\|_{\mathbb{L}_{p}[a, b]}=\left\|\Psi^{*}\right\|_{\mathbb{L}_{p}[0, b-a]}$.

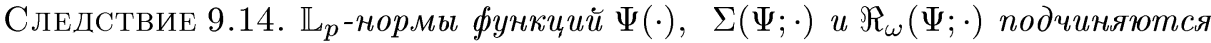
неравенствам

$$
\|\Psi\|_{\mathbb{L}_{p}[a, b]} \leqslant\|\Sigma(\Psi ; \cdot)\|_{\mathbb{L}_{p}[0, b-a]}, \quad\|\Psi\|_{\mathbb{L}_{p}[a, b]} \leqslant\left\|\Re_{\omega}(\Psi ; \cdot)\right\|_{\mathbb{L}_{p}[0, b-a]} .
$$

ЗАмЕчАниЕ 9.15. В [7] автор может найти пример ядра $\Psi \in \mathscr{M}_{4}^{0}[a, b]$ со свойством $\|\Sigma(\Psi ; \cdot)\|_{\mathbb{L}_{1}[a, b]}<\left\|\Re_{\omega}(\Psi ; \cdot)\right\|_{\mathbb{L}_{1}[a, b]}$ и $\|\Sigma(\Psi ; \cdot)\|_{\mathbb{L}_{p}[a, b]}>\left\|\Re_{\omega}(\Psi ; \cdot)\right\|_{\mathbb{L}_{p}[a, b]}$ для всех $p>p_{0}>0$.

Предельные отношения в следуюшем результате немедленно следуют из единственности решений задачи $(\star)$ для $\psi \in \mathscr{M}_{l}^{0}[a, b]$.

ПРЕДЛОЖЕНИЕ 9.16. Пусть $\Psi \in \mathscr{M}_{l}^{0}[a, b] u \Psi_{j} \in \mathscr{M}_{n_{j}}^{0}[a, b]$ для некоторых $n_{j} \leqslant k, j \in \mathbb{N}$. Если $\lim _{j \rightarrow \infty} \Psi_{j}^{\prime}=\Psi^{\prime}$ по мере на $[a, b]$ или если $\lim _{j \rightarrow \infty} \Psi_{j}=\Psi$ в $C[a, b]$, то $\lim _{j \rightarrow \infty} \Re_{\omega}\left(\Psi_{j} ; \cdot\right)=\Re_{\omega}(\Psi ; \cdot)$ поточечно на $[0, b-a]$.

9.5. Неравенство для производных $\omega$-перестановок. Для п.в. $x \in$ $[0, b-a]$ мы построили в [7] такое семейство неперекрывающихся интервалов $\left\{\left[\tau_{i}(x), t_{i}(x)\right]\right\}_{i=1}^{l_{x}}$, что $t_{i}(x)-\tau_{i}(x)=x, \Psi\left(\tau_{i}(x)\right)=\Psi\left(t_{i}(x)\right), i=1, \ldots, l_{x}$, и

$$
\frac{d}{d x} \Re_{\omega}(\Psi ; x)=\sum_{i=1}^{l_{x}}\left[\left|\Psi^{\prime}\left(t_{i}(x)\right)\right|^{-1}+\left|\Psi^{\prime}\left(\tau_{i}(x)\right)\right|^{-1}\right]^{-1} .
$$

ПРЕДЛОЖЕНИЕ 9.17. Если $\Psi \in \mathbb{A C}^{2}[a, b] \cap \mathscr{M}_{l}^{0}[a, b] u \Psi^{\prime \prime} \in \mathscr{M}_{n}^{0}[a, b]$ для некоторых $n, l \in \mathbb{N}$, то

$$
\left|\Re_{\omega}^{\prime}(\Psi ; x)\right| \leqslant \frac{1}{4} \int_{0}^{x} \Sigma\left(\Psi^{\prime \prime} ; x\right) d x, \quad x \in(0, b-a] .
$$

ЗАмечаниЕ 9.18. Эти неравенства точны в том смысле, что существуют ядра $\Psi$, трансформируюшие (9.10) в равенство (см. [4]).

Доказательство (9.10) в [7] является вариацией рассуждения Корнейчука при проверке неравенства

$$
\left|\Sigma^{\prime}(\Psi ; x)\right| \leqslant \frac{1}{4} \int_{0}^{x} \Sigma\left(\Psi^{\prime \prime} ; x\right) d x, \quad x \in(0, b-a],
$$

которое является основньм элементом в дедукции точных оценок сверху для поперечников периодических классов $W^{r} H^{\omega}[-\pi, \pi]$ (см. [19], [20]). 


\section{$\S 10$. Приложения в теории оптимального управления: задача Фельдбаума-Бушоу и тотальный контроль}

\section{1. Области допустимых управлений.}

ОПРЕДЕЛЕНИЕ 10.1. В классической теории оптимального управления область допустимых управлений представляет собой многозначную функцию $U: t \mapsto U(t)$, отображающую интервал $\left[t_{0}, t_{1}\right]$ на непустые подмножества в $\mathbb{R}^{r}$. Допустимый контроль определяется как измеримая селекиия $u(\cdot)$ для $U$, т.е. измеримая функция $u(\cdot)$, удовлетворяющая включениям

$$
u(t) \in U(t) \quad \text { для п.в. } t \in\left[t_{0}, t_{1}\right] .
$$

Среди типичных примеров отметим такие автономные области управлений, как параллелепипеды

$$
\mathbb{P}_{a, b}^{r}:=\left\{\left(u_{1}, u_{2}, \ldots, u_{r}\right) \mid a_{i} \leqslant u_{i} \leqslant b_{i}, i=1, \ldots, r\right\}
$$

и более общие выпуклые множества (см. [7]).

10.2. Задача Фельдбаума-Бушоу. В пятидесятых годах Д. Бушоу [39] и А. А. Фельдбаум [29] предложили математическое решение следующей простейшей задачи быстродействия (см. [9]). Эти решения послужили начальной точкой в построении классической теории оптимального контроля.

Рассмотрим механизм (машину или тележку) единичной массы $m=1$, движушийся без трения по горизонтальной прямой под действием ограниченной управляющей силы $u(t):|u(t)| \leqslant 1$. Координата механизма $x(t)$ в момент времени $t$ определяется вторым законом Ньютона:

$$
m \ddot{x}(t)=u(t) .
$$

Предполагая, что в начальный момент времени машина находится в состоянии покоя (т.е. $x(0)=\dot{x}(0)=0$ ), мы рассмотрим задачу о наибыстрейшем попадании механизма в заданную точку $x(T)=x_{0}, \dot{x}(T)=x_{1}$ фазовой плоскости и об определении экстремального контроля $\hat{u}(t)$, осуществляющего это управление.

ОПРЕДЕЛЕниЕ 10.2. Для $\tau \in[a, b]$ onepamop $\mathbb{I}_{\tau}^{n}: C^{n}[a, b] \rightarrow \mathbb{R}^{n+1}, f \mapsto \mathbb{I}_{\tau}^{n}(f)$, вводится следуюшим образом:

$$
\mathbb{I}_{\tau}^{n}(f):=\left(\lambda_{0}(f), \lambda_{1}(f), \ldots, \lambda_{n}(f)\right),
$$

где $\lambda_{i}(f)=f^{(i)}(\tau), i=0, \ldots, n$.

Тогда более общая задача Фельдбаума-Бушоу может быть сформулирована следуюшим образом:

$$
T \rightarrow \inf ; \quad x \in W_{\infty}^{r+1}[0, T], \quad \mathbb{I}_{0}^{r}(x)=\mathbf{0}, \quad \mathbb{I}_{T}^{r}(x)=\Gamma,
$$

где $\Gamma \in \mathbb{R}^{r+1}$ и $\mathbf{0}$ - нуль в $\mathbb{R}^{r+1}$. 
10.3. Принцип максимума в задаче Фельдбаума-Бушоу. Пусть $(\hat{x}, \hat{u}, \widehat{T})$ - оптимальный процесс для задачи (10.4). Принцип максимума Понтрягина (см. [27]) гарантирует существование таких кусочно постоянных $C[0, \widehat{T}]$-функций $\left\{p_{k}(\cdot)\right\}_{k=1}^{r}$, что следуюшие условия удовлетворены для

$$
L(t, x(t), \dot{x}(t), u(t)):=\lambda+\sum_{k=1}^{r} p_{k}(t)\left(\dot{x}_{k}(t)-x_{k+1}(t)\right)+p_{r+1}(t)\left(\dot{x}_{r+1}(t)-u(t)\right),
$$

лагранжиана задачи (10.4):

i) каноничекие уравнения Эйлера $-\frac{d}{d t} L_{\dot{x}_{k}}(t)+L_{x_{k}}(t)=0, k=1, \ldots, r+1$ :

$$
\dot{p}_{1}(t)=0, \quad \dot{p}_{k+1}(t)=-p_{k}(t), \quad k=1, \ldots, r .
$$

ii) условие Вейеритрасса $\min _{u(t) \in U} L(t, \hat{x}(t), \dot{\hat{x}}(t), u(t))=L(t, \hat{x}(t), \dot{\hat{x}}(t), \hat{u}(t))$ :

$$
\max _{u(t) \in[-1,1]} p_{r+1}(t) u(t)=p_{r+1}(t) \hat{u}(t) \quad \text { для п.в. } t \in[0, \widehat{T}] .
$$

Из (10.5) следует, что $p_{r+1}(t)=\sum_{i=0}^{r} \alpha_{i} t^{i}$ является многочленом степени $r$, а условие Вейерштрасса (10.6) требует того, чтобы оптимальньй контроль $\hat{u}(t)$ совпадал со знаком $p_{r+1}(t)$ для п.в. $t$, где $p_{r+1}(t) \neq 0$.

Необходимые условия (10.5), (10.6) принципа максимума являются также достаточныци (см. [7], [47] или элементарное прямое доказательство в [5]) для описания экстремалей задачи (10.4).

Teорема 10.3. Для любого вектора $\Gamma=\left(\gamma_{0}, \ldots, \gamma_{r}\right) \in \mathbb{R}^{r+1} \backslash\{0\}$ существуют такие $T>0$ и множество $k \leqslant r$ точек $\left\{\tau_{i}\right\}_{i=1}^{k}: 0<\tau_{1}<\tau_{2}<\cdots$ $\cdots<\tau_{k}<T$ и $\xi \in\{ \pm 1\}$, ито функиия

$$
H_{T, \Gamma}(t)=\frac{\xi t^{r+1}}{(r+1) !}+\frac{2 \xi}{(r+1) !} \sum_{i=1}^{k}(-1)^{i}\left(t-\tau_{i}\right)_{+}^{r+1}
$$

удовлетворяет граничныц условиям $\mathbb{I}_{T}^{r}\left(H_{T, \Gamma}\right)=\Gamma$. Функция $H_{T, \Gamma}-$ единственная әкстремаль в (10.4).

Теорема 10.3 описывает и рис. 10.1 иллюстрирует семейство оптимальных траекторий задачи (10.4) в классическом случае $r=1$.

Если точка $\left(\Gamma_{0}, \Gamma_{1}\right)$ лежит на кривой $\Upsilon_{-}: x=(1 / 2) \dot{x}^{2}, \dot{x} \leqslant 0 \quad\left(\Upsilon_{+}: x=\right.$ $\left.-(1 / 2) \dot{x}^{2}, \dot{x} \geqslant 0\right)$, то $u^{*}=-1\left(u^{*}=1\right)$, оптимальное движение $(x(t), \dot{x}(t))$ проходит по кривой $\Upsilon_{-}\left(\Upsilon_{+}\right)$. Если точка $\left(\Gamma_{0}, \Gamma_{1}\right)$ принадлежит области над (под) кривой переключений $\Upsilon:=\Upsilon_{-} \cup \Upsilon_{+}$, то она лежит на кривой $\Upsilon_{c}^{+}: x=c-(1 / 2) \dot{x}^{2}$ $\left(\Upsilon_{c}^{-}: x=(1 / 2) \dot{x}^{2}-c\right)$ для некоторого $c>0$, и оптимальная траектория в фазовых координатах состоит из движения по $\Upsilon_{-}\left(\Upsilon_{+}\right)$с замедлением -1 (ускорением 1$)$ 
с последующим переключением на параболу $\Upsilon_{c}^{+}\left(\Upsilon_{c}^{-}\right)$в точке $(c / 2, \sqrt{c} / 2)$ пересечения двух кривых и движением с ускорением 1 (замедлением -1 ).

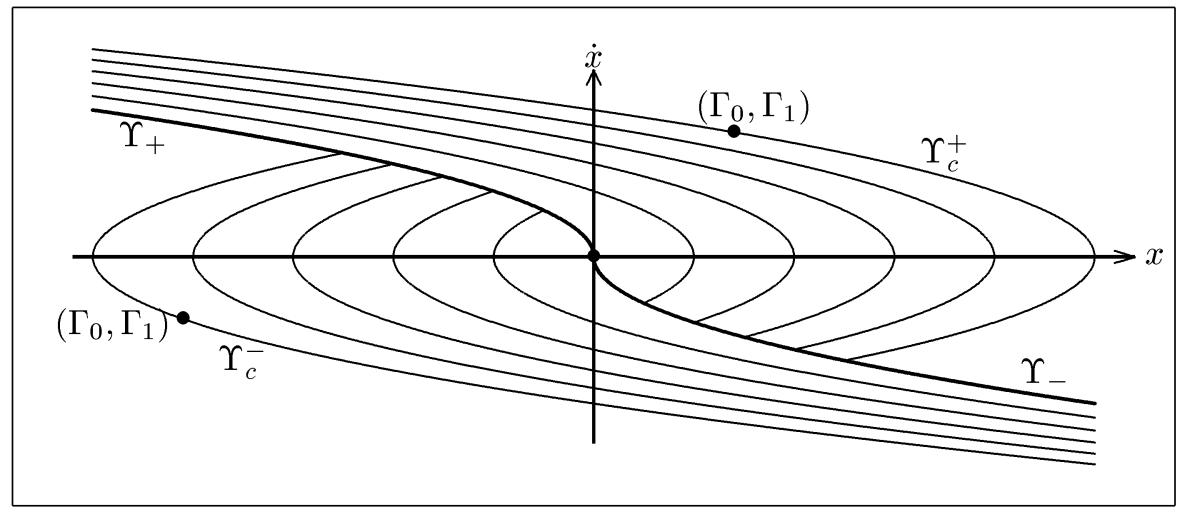

Рис. 10.1. Оптимальные траектории задачи Фельдбаума-Бушоу

10.4. Области управлений, контроли в теории тотального контроля. Если контроль $u(t)$ удовлетворяет ограничению $\alpha \leqslant u(t) \leqslant \beta, t \in\left[t_{0}, t_{1}\right]$, $\alpha<0<\beta$, то переменная управления $v(t)=\int_{t_{0}}^{t} u(s) d s$ удовлетворяет условию Липшица

$$
\alpha\left(t^{\prime \prime}-t^{\prime}\right) \leqslant v\left(t^{\prime \prime}\right)-v\left(t^{\prime}\right) \leqslant \beta\left(t^{\prime \prime}-t^{\prime}\right), \quad t_{0} \leqslant t^{\prime}<t^{\prime \prime} \leqslant t_{1} .
$$

В наших обобщениях мы заменим линейные модули непрерывности $\omega_{1}(t)=-\alpha t$ и $\omega_{2}(t)=\beta t$ на произвольные выпуклые модули непрерывности и рассмотрим управления, подчиняющиеся ограничениям

$$
-\omega_{1}(|t-\tau|) \leqslant u(t)-u(\tau) \leqslant \omega_{2}(|t-\tau|), \quad t, \tau \in\left[t_{0}, t_{1}\right]
$$

В отличие от линейного случая $\omega(t)=t$ включения (10.9) определяют множество контролей, которое не может считаться областью управления в классическом смысле определения 10.1. Таким образом, для решения таких задач необходимо ввести новые понятия области управления $U$, допустимых контролей $u(\cdot)$, и построить новую теорию для этих контролей.

ОПРЕДЕЛЕНИЕ 10.4. В теории тотального контроля областью управления называется такая многозначная функция $U:(t, \tau, u) \mapsto U(t, \tau, u)$, отображающая $\left[t_{0}, t_{1}\right] \times\left[t_{0}, t_{1}\right] \times \mathbb{R}^{r}$ на непустые множества в $\mathbb{R}^{r}$, что

$$
U(t, t, u)=u \quad \forall t \in\left[t_{0}, t_{1}\right], \quad \forall u \in \mathbb{R}^{r}
$$

Контролем, или допустимылм управлением, называется измеримая селекция $u(\cdot)$ для $U$, удовлетворяющая включениям

$$
u(t) \in \bigcap_{\tau \in\left[t_{0}, t_{1}\right]} U(t, \tau, u(\tau)) \quad \forall t \in\left[t_{0}, t_{1}\right]
$$


Заметим, что теория тотального контроля включает в себя классическую теорию оптимального контроля, в которой области управлений $U$ не зависят от $\tau$ и $u$.

Заметим, что подмножества $U(t, \tau, u)$, порождающие допустимые контроли $u(\cdot)$ в (10.9), являются замкнутыми интервалами

$$
U(t, \tau, u):=[u-\omega(|t-\tau|), u+\omega(|t-\tau|)], \quad t, \tau \in\left[t_{0}, t_{1}\right], \quad u \in \mathbb{R} .
$$

Выбирая обобщенную задачу Фельдбаума-Бушоу в качестве достаточно простого и в то же время типичного примера проблемы из теории тотального контроля и опираясь в нашем обзоре на результаты работы [6], мы укажем новую версию необходимых условий оптимальности в виде интегрального приниипа максимума и модифицируем принцип динамического программирования Беллмана.

10.5. Обобщенная задача Фельдбаума-Бушоу. Обобщенная задача Фельдбаума-Бушоу формулируется следующим образом:

$$
T \rightarrow \inf ; \quad \mathbb{I}_{0}^{r}(x)=\mathbf{0}, \quad \mathbb{I}_{T}^{r}(x)=\Gamma, \quad x \in W^{r} H^{\omega}[0, T],
$$

для всех $r \in \mathbb{N}, \quad \Gamma \in \mathbb{R}^{r+1}$ и выпуклых модулей непрерьвности $\omega$. Заметим, что задача Фельдбаума-Бушоу $(10.4)$ в соболевском классе $W_{\infty}^{r+1}[0, T]$ является частным случаем проблемы (10.13) в гельдеровском классе $W^{r} H^{1}[0, T]$. Следовательно, необходимые условия оптимальности функции $\hat{x}$ в $(10.13)$ можно выразить в следующей эквивалентной форме интегрального условия Вейерштрасса і) и уравнений Эйлера ii):

i) $\int_{0}^{\widehat{T}} \hat{x}^{(r)}(t) q(t) d t=\max _{x \in \mathscr{W}_{\Gamma_{r}}^{r}[0, \widehat{T}]} \int_{0}^{\widehat{T}} x^{(r)}(t) q(t) d t$,

ii) $q^{(r)}(t) \equiv 0$,

где $\Gamma=\left(\Gamma_{0}, \ldots, \Gamma_{r}\right), \mathscr{W}_{M}^{r}[0, T]:=\left\{x \in W^{r} H^{1}[0, T] \mid x^{(r)}(0)=0, x^{(r)}(T)=M\right\}$.

Естественно было бы ожидать, что аналогичные условия экстремальности (при замене $W^{r} H^{1}[0, \widehat{T}]$ в $\left.(10.14), \mathrm{i}\right)$ на $\left.W^{r} H^{\omega}[0, \widehat{T}]\right)$ могут быть получены для задачи (10.13). Однако результаты, приведенные в следующем пункте, демонстрируют, что принцип максимума Понтрягина в форме канонических уравнений Эйлера и интегрального условия Вейерштрасса недостаточны для описания необходимых условий оптимальности для всех решений задачи (10.13).

\section{6. Обозначения и определения.}

ОПРЕДЕЛЕНИЕ 10.5. Для $m \in \mathbb{N}$ пусть $\mathbb{S}^{m}-$ единичная сффера в $l_{1}^{m+1}$ :

$$
\mathbb{S}^{m}:=\left\{s=\left(s_{0}, \ldots, s_{m}\right)\left|\sum_{i=0}^{m}\right| s_{i} \mid=1\right\} .
$$

Множество $\mathscr{P}_{r}$ нормализованных многочленов степени $r-1$ вводится следуюшим образом:

$$
\mathscr{P}_{r}:=\left\{p_{s}(t):=\sum_{i=0}^{r-1} s_{i} t^{i} \mid s=\left(s_{0}, s_{1}, \ldots, s_{r-1}\right) \in \mathbb{S}^{r-1}\right\}, \quad r \in \mathbb{N} .
$$


ОПРЕДЕЛЕНИЕ 10.6. Пусть $s \in \mathbb{S}^{r-1}, T>0$ и $\xi \in[-\omega(T), \omega(T)]$. Тогда $h_{s, T, \xi}(\cdot)$ определяется на $[0, T]$ как экстремаль задачи

$$
\int_{0}^{T} h(t) p_{s}(t) d t \rightarrow \sup , \quad h \in H^{\omega}[0, T], \quad h(0)=0, \quad h(T)=\xi .
$$

Строение экстремальных функций в (10.16) описано в теоремах 7.13 и 7.8.

ОПРЕДЕЛЕНИЕ 10.7. Пусть $s \in \mathbb{S}^{r-1}, T>0$ и $\xi \in[-\omega(T), \omega(T)]$. Тогда $H_{s, T, \xi}(\cdot)$ - следуюший $r$-й интеграл функции $h_{s, T, \xi}$ :

$$
H_{s, T, \xi}(x)=\frac{1}{(r-1) !} \int_{0}^{T}(x-t)_{+}^{r-1} h_{s, T, \xi}(t) d t, \quad x \in[0, T] .
$$

ОПРЕДЕЛЕНИЕ 10.8. Пусть $s \in \mathbb{S}^{r-1}, T>0$ и $\xi \in[-\omega(T), \omega(T)]$. Тогда вектор $\Gamma_{s, T, \xi} \in \mathbb{R}^{r+1}$ әраничных условий определяется посредством

$$
\Gamma_{s, T, \xi}=\left(\Gamma_{0}, \Gamma_{1}, \ldots, \Gamma_{r}\right), \quad \Gamma_{k}:=H_{s, T, \xi}^{(k)}(T), \quad k=0, \ldots, r .
$$

В терминах обозначения $10.2 \Gamma_{s, T, \xi}:=\mathbb{I}_{T}^{r}\left(H_{s, T, \xi}\right)$.

Следуюший результат показывает, что если $\Gamma=\Gamma_{s, T, \xi}$ для тройки $(s, T, \xi)$, то $H_{s, T, \xi}$ - единственное решение задачи (10.13).

ПРЕДЛОЖЕНИЕ 10.9. Пусть $s \in \mathbb{S}^{r-1}, T>0 u \xi \in[-\omega(T), \omega(T)]$. Функиия $H_{s, T, \xi}$ является единственной экстремалью задачи (10.13) для $\Gamma=\Gamma_{s, T, \xi}$.

Согласно теореме 10.3 , если $\omega$ - линейный модуль непрерывности, каждый вектор $\Gamma \in \mathbb{R}^{r+1}$ совпадает с некоторым $\Gamma_{s, T, \xi}$. Однако в случае нелинейны $x$ модулей непрерьвности $\omega$ функции $H_{s, T, \xi}$ не исчерпьвают множество экстремалей задачи (10.13).

ОПРЕДЕЛЕНИЕ 10.10. Пусть $T>0, \gamma \in\{ \pm 1\}$ и $\psi \in \mathbb{L}[0, T]-$ ядро с конечным числом точек перемены знака в смысле определения 7.4. Через $h_{\psi, T, \gamma}$ обозначим экстремаль задачи

$$
\int_{0}^{T} h(t) \psi(t) d t \rightarrow \sup , \quad h \in H^{\omega}[0, T], \quad h(0)=0, \quad h(T)=\gamma \omega(T) .
$$

Функция $H_{\psi, T, \gamma}$ является $r$-м интегралом $h_{\psi, T, \gamma}$, удовлетворяющим нулевым граничным условиям в нуле:

$$
H_{\psi, T, \gamma}(x)=\frac{1}{(r-1) !} \int_{0}^{T}(x-t)_{+}^{r-1} h_{\psi, T, \gamma}(t) d t, \quad x \in[0, T],
$$

а вектор $\Gamma_{\psi, T, \gamma}$ определяется посредством

$$
\Gamma_{\psi, T, \gamma}:=\mathbb{I}_{T}^{r}\left(H_{\psi, T, \gamma}\right) .
$$

В соответствии со следуюшим результатом если $r$-я производная достигает максимального $\omega(T)$ или минимального $-\omega(T)$ значения за время $T$, то $H_{\psi, T, \gamma}$ экстремальна в (10.13) вне зависимости от функции $\psi$. 
ПРЕДЛОЖЕНИЕ 10.11. Пусть $T>0, \gamma \in\{ \pm 1\} u \psi \in \mathbb{L}[0, T]$ - ядро с конечным числом точек перемены знака на $[0, T]$. Тогда $H_{\psi, T, \gamma}$ әкстремальна в задаче (10.13) для $\Gamma=\Gamma_{\psi, T, \gamma}$.

Оказывается, что предложения 10.9 и 10.11 описывают все оптимальные траектории задачи (10.13).

10.7. Управляемость и оптимальные траектории. В нашем описании необходимых и достаточных условий экстремальности в (10.13) мы дадим две альтернативы уравнениям Эйлера и условиям Вейерштрасса классической теории оптимального управления.

Первый результат гарантирует, что для любого вектора $\Gamma \in \mathbb{R}^{r+1}$ или $\Gamma=\Gamma_{s, T, \xi}$ и $H_{s, T, \xi}$ - единственная экстремаль в задаче (10.13) для некоторого $(s, T, \xi)$, или $\Gamma=\Gamma_{p, T, \gamma}$ для полинома степени $r$.

Tеорема 10.12. Пусть $\lim _{t \rightarrow \infty} \omega(t)=\infty$. Для любого $\Gamma \in \mathbb{R}^{r+1} \backslash\{\mathbf{0}\}$ справедливо одно из следующих двух утверждений:

1) существуют такие $s \in \mathbb{S}^{r}, T>0 u \xi \in \mathbb{R}$, что $\Gamma=\Gamma_{s, T, \xi}$ и функиия $H_{s, T, \xi}$ әкстремальна в задаче (10.13);

2) существуют такие $p(t)=\sum_{i=0}^{r} \alpha_{i} t^{i}, T>0 u \gamma \in\{ \pm 1\}$, что $\Gamma=\Gamma_{p, T, \gamma}$, и функиия $H_{p, T, \gamma}$ әкстремальна в задаче (10.13).

Другое описание решений задачи (10.13) показывает, что для любого ненулевого вектора $\Gamma \in \mathbb{R}^{r+1}$ сушествуют такие две тройки $\left(s_{1}, T, \xi\right)$ и $\left(s_{2}, T, \xi\right)$, что $\Gamma$ является вьпуклой комбинацией $\Gamma_{s_{1}, T, \xi}$ и $\Gamma_{s_{2}, T, \xi}$, и соответствуюшая экстремаль задачи (10.13) является выпуклой комбинацией функций $H_{s_{1}, T, \xi}$ и $H_{s_{2}, T, \xi}$.

TEOPEMA 10.13. Пусть $\lim _{t \rightarrow \infty} \omega(t)=\infty$. Для любого $\Gamma \in \mathbb{R}^{r+1} \backslash\{0\}$ справедливо одно из следующих двух утверждений:

1) существуют такие $s \in \mathbb{S}^{r}, T>0 u \xi \in \mathbb{R}$, ито $\Gamma=\Gamma_{s, T, \xi}$;

2) существуют такие $s_{1}, s_{2} \in \mathbb{S}^{r}, T \geqslant 0, \zeta \in\{ \pm 1\}$ u $\alpha \in(0,1)$, ито

$$
\Gamma=\alpha \Gamma_{s_{1}, T, \zeta \omega(T)}+(1-\alpha) \Gamma_{s_{2}, T, \zeta \omega(T)},
$$

и функиия $\alpha H_{s_{1}, T, \zeta \omega(T)}+(1-\alpha) H_{s_{2}, T, \zeta \omega(T)}$ әкстремальна в задаче (10.13).

10.8. Принцип динамического программирования. Пусть $\widehat{T}-$ минимальное время и $\hat{x}(t), 0 \leqslant t \leqslant \widehat{T},-$ оптимальное решение задачи (10.4). Принuип динамического программирования Беллмана (см. [36]) для задачи (10.4) утверждает, что любой участок экстремальной траектории также оптимален.

ПРЕДЛОЖЕНИЕ 10.14. Пусть $Г(\tau):=\mathbb{I}_{\tau}^{r}(\hat{x}), \quad \tau \in(0, \widehat{T})$. Тогда $\tau$ u $\widehat{T}-\tau$ являются минимальными временами и $\left.\hat{x}\right|_{[0, \tau]}(\cdot),\left.\hat{x}\right|_{[\tau, \widehat{T}]}(\cdot-\tau)$ әкстремальны в задачах

$$
\begin{array}{llll}
T \rightarrow \text { inf; } & x \in W_{\infty}^{r+1}[0, T], & \mathbb{I}_{0}^{r}(x)=\mathbf{0}, & \mathbb{I}_{T}^{r}(x)=\Gamma(\tau), \\
T \rightarrow \text { inf; } & x \in W_{\infty}^{r+1}[0, T], & \mathbb{I}_{0}^{r}(x)=\Gamma(\tau), & \mathbb{I}_{T}^{r}(x)=\Gamma
\end{array}
$$

соответственно для всех $\tau \in(0, \widehat{T})$. 
Результаты п. 7.6 в сочетании с теоремой 10.13 означают, что существует только конечное число точек $\tau$ с тем свойством, что оба куска $\left.\hat{x}\right|_{[0, \tau]}$ и $\left.\hat{x}\right|_{[\tau, \widehat{T}]}$ траектории экстремальны в соответствующих задачах быстродействия. Более точно, пусть $\hat{x}$ является решением задачи (10.13), $\widehat{T}$ - минимальное время и $\Gamma(\tau):=\mathbb{I}_{\tau}^{r}(\hat{x})$, $\tau \in[0, \widehat{T}]$. Пусть также $p$ - порождающий полином (степени $r-1$ или $r)$ для $\hat{x}^{(r)}$.

ПРЕДЛОЖЕнИЕ 10.15. Пусть $\tau \in(0, \widehat{T})$. Тогда $\tau, \widehat{T}-\tau$ являются минимальныци временами и $\left.\hat{x}\right|_{[0, \tau]}(\cdot),\left.\quad \hat{x}\right|_{[\tau, T]}(\cdot-\tau)$ әкстремальны в задачах

$$
\begin{array}{llll}
T \rightarrow \text { inf; } & x \in W^{r} H^{\omega}[0, T], & \mathbb{I}_{0}^{r}(x)=\mathbf{0}, & \mathbb{I}_{T}^{r}(x)=\Gamma(\tau), \\
T \rightarrow \text { inf; } & x \in W^{r} H^{\omega}[0, T], & \mathbb{I}_{T}^{r}(x)=\Gamma(\tau), & \mathbb{I}_{T}^{r}(x)=\Gamma
\end{array}
$$

соответственно тогда и только тогда, когда

$$
\begin{aligned}
& \int_{0}^{\tau} \hat{x}^{(r)}(t) p(t) d t=\sup \left\{\int_{0}^{\tau} h(t) p(t) d t \mid h \in H^{\omega}[0, \tau], h(0)=0\right\} \\
& \int_{\tau}^{\widehat{T}} \hat{x}^{(r)}(t) p(t) d t=\sup \left\{\int_{\tau}^{\widehat{T}} h(t) p_{\Gamma}(t) d t \mid h \in H^{\omega}[\tau, \widehat{T}], h(\widehat{T})=\Gamma_{r}\right\} .
\end{aligned}
$$

Мы охарактеризовали такие точки Беллмана $\tau$ в замечании 7.14 и показали, что общее число этих точек $\tau$ не превосходит $r+1$, поскольку многочлены степени $r$ могут иметь не более $r$ нулей. В [6] мы идентифицируем эти точки для задачи (10.13) в элементарных случаях $r=1$ и $r=2$. В [6] и [34] мы обсуждаем разнообразные свойства множеств достижимости, или сфер Беллмана, и раскрываем замечательные свойства экстремалей задачи (10.13) в гельдеровых классах.

10.9. Общая линейная задача быстродействия. Теория следующей общей задачи быстродействия разрабатывается в [34]:

$$
\begin{gathered}
T \rightarrow \inf ; \quad x(0)=0, \quad x(T)=\Gamma, \\
\dot{x}(t)=A(t) x(t)+B(t) u(t), \quad u(\cdot) \in H^{\omega_{1}}[0, T] \times \cdots \times H^{\omega_{m}}[0, T],
\end{gathered}
$$

где $x(t), \Gamma \in \mathbb{R}^{n}, u(t) \in \mathbb{R}^{m}, A(t)$ и $B(t)-(n \times n)$ - и $(n \times m)$-матрицы и $\left\{\omega_{i}\right\}_{i=1}^{m}-$ вьпуклые модули непрерывности. В случае $\omega(t)=t$ задача (10.23) для постоянных матриц $A$ (с собственными значениями в левой полуплоскости) и $B$ получила свое решение в [37]. Детальное обсуждение обшей теории линейных задач быстродействия с полигональными областями допустимых управлений можно найти в [7] или [47].

10.10. Задача Колмогорова-Ландау в $\mathbb{L}_{p}$. Общая задача Колмогорова-Ландау в $\mathbb{L}_{p}\left(\mathbb{R}_{+}\right)$и $\mathbb{L}_{p}(\mathbb{R})$ представляет типичный пример задачи нелинейного тотального контроля:

$$
\left\|f^{(m)}\right\|_{\mathbb{L}_{\infty}(\mathbb{I})} \rightarrow \sup , \quad f \in W^{r} H^{\omega}(\mathbb{I}), \quad\|f\|_{\mathbb{L}_{p}(\mathbb{I})} \leqslant B,
$$

где $\mathbb{I}=\mathbb{R} \vee \mathbb{R}_{+}, B>0,1 \leqslant p \leqslant+\infty$ и $0 \leqslant m \leqslant r$ при $p<\infty$ и $0<m \leqslant r$ при $p=\infty$. Мы упомянем некоторые результаты из [35], иллюстрирующие приложения интегрального принципа максимума в нелинейной задаче (10.24). 
Teоpema 10.16. Пусть $r, m \in \mathbb{N}: 0 \leqslant m<r ; \quad B>0, \quad p \geqslant 1 u \omega-$ выпуклый модуль непрерывности.

1. Существуют такие функции $X \in W^{r} H^{\omega}\left(\mathbb{R}_{+}\right)$и $Z$, что:

i) $Z^{(r+1)}(t)=|X(t)|^{p-1} \operatorname{sign} X(t), \quad t \in \mathbb{R}_{+}$;

ii) $\int_{\mathbb{R}_{+}} X^{(r)}(t) Z^{\prime}(t) d t=\sup _{h \in H^{\omega}\left(\mathbb{R}_{+}\right)} \int_{\mathbb{R}_{+}} h(t) Z^{\prime}(t) d t$;

iii) $Z^{(i)}(0)=\delta_{i, r-m}, \quad i=0, \ldots, r$;

iv) $\|X\|_{\mathbb{L}_{p}\left(\mathbb{R}_{+}\right)}=B$.

2. Для любой функции $f \in W^{r} H^{\omega}\left(\mathbb{R}_{+}\right):\|f\|_{\mathbb{L}_{p}\left(\mathbb{R}_{+}\right)} \leqslant B$ справедливо следующее неравенство:

$$
\left\|f^{(m)}\right\|_{\mathbb{L}_{\infty}\left(\mathbb{R}_{+}\right)} \leqslant X^{(m)}(0)=\left|Z^{(r-m)}(0)\right|^{-1}\left[B^{p+1}+\int_{\mathbb{R}_{+}} \Re_{\omega}(Z ; t) \omega^{\prime}(t) d t\right] .
$$

Условия оптимальности включают в себя уравнения Эйлера (10.25), i), интељральный вариант условия Вейеритрасса (10.25), ii) и условие трансверсальности (10.25), iii) (см. [25]). Как и в [25] в случае $\omega(t)=t$, в [35] мы находим точные

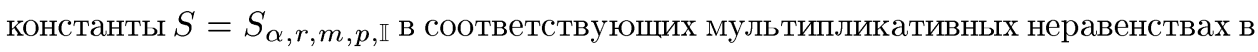
гельдеровых классах $W^{r} H^{\alpha}(\mathbb{I}), \mathbb{I}=\mathbb{R} \vee \mathbb{R}_{+}$:

$$
\left\|f^{(m)}\right\|_{\mathbb{L}_{\infty}(\mathbb{I})} \leqslant S\|f\|_{\mathbb{L}_{p}(\mathbb{I})}^{\frac{r+\alpha-m}{r+\alpha+p^{-1}}} \quad \forall f \in W^{r} H^{\alpha}(\mathbb{I}) .
$$

Мы также упомянем замечательное свойство экстремальных функций $X_{B}$ задачи (10.24) в случае $r=m=1, B>0, \mathbb{I}=\mathbb{R}_{+}$гельдеровых модулей $\omega(t)=t^{\alpha}$, $0<\alpha \leqslant 1$. В этом случае набор узлов $\left\{\xi_{i}\right\}_{i=1}^{\infty}$ (точек перемены знака функции $X_{B}^{\prime \prime}$ ) образует геометрическое разбиение:

$$
\frac{\xi_{i+1}-\xi_{i}}{\xi_{i}-\xi_{i-1}}=\gamma, \quad \gamma=\gamma(\alpha) \in(0,1), \quad i \in \mathbb{N},
$$

где $\xi_{0}:=0$. В частности, носителем функции $X_{B}$ является ограниченный интервал $[0, d]$. Более того, $X_{B}$ имеет свойство подобия, свойственное фракталам: для всех $i \in \mathbb{N}$

$$
X_{B}\left(t+\xi_{i}\right)=(-1)^{i}\left(\frac{\xi_{i+1}-\xi_{i}}{\xi_{1}}\right)^{1+\alpha} X_{B}\left(\frac{\xi_{1}}{\xi_{i+1}-\xi_{i}} t\right), \quad t \in\left[0, \xi_{i+1}-\xi_{i}\right] .
$$

Этот феномен в случае $\omega(t)=t$ и $p=2$ был открыт А. Т. Фуллером [44], а также В. Н. Габушиным [8] и позже Г. Г. Магарил-Ильяевым [24] для всех $p \in[1,+\infty)$.

В заключении нашего краткого введения в теорию тотального контроля мы подчеркнем, что интегральный принцип максимума для экстремальных задач для областей управления $U(t)$ классической теории обсуждались Л.С. Янгом [65] и Р.В. Гамкрелидзе [10], установивших эквивалентность "интегрального" и "поточечного" вариантов принципа максимума для таких контролей. В работах автора [6], [34] и [35] на примере конкретных задач показано, что в теории тотального контроля әкстремальное управление максимизирует интеграл $\int_{t_{0}}^{t_{1}} h(t, x(t), \dot{x}(t)) d t$ гамильтониана системь. 


\section{Список литературы}

1. Ахиезер Н. И. Лекции по теории аппроксимации. М.: Наука, 1965.

2. Багдасаров С. К. Максимизация функционалов в $H^{\omega}[a, b] / /$ Матем. сб. 1998. Т. 189. № 2. C. 3-72.

3. Багдасаров С. К. Общая конструкция чебышевских $\omega$-сплайнов данной нормы // Алгебра и анализ. 1998. Т. 8. №6.

4. Багдасаров C. К. Свойства $\omega$-сплайнов // Функц. анализ и его прилож. 1998 (в печати).

5. Багдасаров C. К. Элементарное решение задачи Фельдбаума-Бушоу // Матем. заметки. 1999 (в печати).

6. Багдасаров C.K. Задача Фельдбаума-Бушоу, интегральный принцип максимума и введение в тотальный контроль // Матем. сб. 1999 (в печати).

7. Болтянский В.Г. Математические методы оптимального управления. М.: Наука, 1969.

8. Габушин $B . H$. Неравенства для норм функции и ее производной в метриках $L_{p} / /$ Матем. заметки. 1967. Т. 1. № 3. С. 291-298.

9. Галеев Э.М., Тихомиров В.M. Краткий курс теории экстремальных задач. М.: Изд-во МГУ, 1989.

10. Гамкрелидзе Р. В. Основы оптимального управления. Тбилиси: Изд-во ТГУ, 1975.

11. Емеличев В.А., Мельников О.И., Сарванов В.И., Тъикевич Р.И. Лекции по теории графов. М.: Наука, 1990.

12. Золотарев Е. И. Приложения эллиптических функций к задаче о функциях, наименее и наиболее уклоняющихся от нуля // Зап. С.-Пб. Ак. наук. 1877. Т. 30. № 5. С. 1-59 (см. также Избранные труды. Т. ІІ. Л.: Изд-во АН СССР, 1932).

13. Канторович Л. В. О перемещении масс // ДАН СССР. 1942. Т. 37. С. 227-229.

14. Канторович Л. В., Акилов Г. П. Функциональный анализ. М.: Наука, 1984.

15. Канторович Л.В., Рубинштейн Г. Ш. Об одном функциональном пространстве и некоторых экстремальных задачах // ДАН СССР. 1957. Т. 115. С. 1058-1061.

16. Колмогоров A. Н. О неравенствах между верхними гранями последовательных производных функций на бесконечных интервалах // Уч. зап. МГУ. Математика. 1939. Т. 3. C. 3-16.

17. Колмогоров A. Н. Избранные труды. Математика и механика. М.: Наука, 1985.

18. Корнейчук Н. П. Экстремальные значения функционалов и наилучшее приближение на классах периодических функций // Изв. АН СССР. Сер. матем. 1971. Т. 35. № 2. C. $423-434$.

19. Корнейчук Н. П. Экстремальные задачи теории приближения. М.: Наука, 1976.

20. Корнейчук Н. П. Сплайны в теории приближения. М.: Наука, 1984.

21. Корнейчук Н. П. Точные константы в теории приближения. М.: Наука, 1990.

22. Корнейчук Н. П. С. М. Никольский и развитие исследований по теории приближения в СССР // УМН. 1985. Т. 40. С. 83-156.

23. Крейн С. Г., Петунин Ю.И., Семенов Е. М. Интерполяция линейных операторов. М.: Наука, 1978.

24. Магарил-Ильяев Г. Г. О колмогоровских неравенствах на полупрямой // Вест. МГУ. Сер. матем. 1976. Т. 31. № 5. С. 33-41.

25. Магарил-Ильяев Г. Г. Неравенства для производных и двойственность // Тр. МИАН. 1983. T. 161. C. $183-194$.

26. Малоземов В. Н., Певныи А. Б. Полиномиальные сплайны. Л.: Изд-во ЛГУ, 1986.

27. Понтрягин Л. С., Болтянский В. Г., Гамкрелидзе Р. В., Мищенко Е. Ф. Математическая теория оптимальных процессов. М.: Наука, 1983. 
28. Тихомиров В. М. Наилучшие методы приближения и интерполяции дифференцируемых функций в пространстве $C[-1,1]$ // Матем. сб. 1969. Т. 9. С. 277-289.

29. Фельдбаум A.А. О синтезе оптимальных систем с помощью фазового пространства // Автоматика и телемеханика. 1955. Т. 16. С. 129-149.

30. Чебышев П. Л. Избранные труды. М.: Изд-во АН СССР, 1955.

31. Bagdasarov S. K. Chebyshev Splines and Kolmogorov Inequalities. Basel: Birkhäuser, 1998.

32. Bagdasarov S. K. Zolotarev $\omega$-polynomials in $W^{r} H^{\omega}[0,1] / /$ J. Approx. Theory. 1997. V. 90. № 3. P. 340-378.

33. Bagdasarov $S$. K. Kolmogorov problem in $W^{r} H^{\omega}[0,1]$ and extremal Zolotarev $\omega$-splines // Dissertationes Mathematicae. 1998. V. 502.

34. Bagdasarov S. K. General time optimal linear problem and integral maximum principle. 1998. (in preparation).

35. Bagdasarov S. K. Kolmogorov problem for intermediate derivatives and optimal control. (in preparation).

36. Bellman R. E. Dynamic Programming. Princeton-N.J.: Princeton Univ. Press, 1957.

37. Bellman R., Glicksberg I., Gross O. On the Bang-Bang control problem // Quart. Appl. Math. 1956. V. 14. P. 11-18.

38. Bennett C., Sharpley R. Interpolation of Operators. N. Y.: Academic Press, Inc., 1988.

39. Bushaw D. Optimal discontinuous forcing terms // Theory of Nonlinear Oscillations. V. 4. Princeton-N. J.: Princeton Univ. Press, 1958. P. 29-52.

40. Cavaretta A. S., Schoenberg I. J. Solution of Landau's problem concerning higher derivatives on the half-line // Proc. of the Intern. Conf. on Constructive Function Theory. Sofia: Publ. House Bulg. Acad. Sci., 1972. P. 297-308.

41. Chong K. M. Some extensions of a theorem of Hardy, Littlewood and Polya and their applications // Can. J .Math. 1974. V. 26. P. 1321-1340.

42. DeVore R.A., Lorentz G. G. Constructive Approximation. Berlin-Heidelberg: Springer-Verlag, 1993.

43. Dorfman R., Samuelson P. A., Solow R. M. Linear Programming and Economic Analysis. N.Y.: Dover Publications, Inc., 1986.

44. Fuller A. T. Optimization of nonlinear control systems with transient inputs // J. of Electron. and Control. 1960. V. 8. № 6. P. 465-479.

45. Hadamard J. Sur le module maximum d'une fonction et de ses dérivées // Soc. math. France. Comptes rendus, des Séances. 1914. V. 41. P. 68-72.

46. Hardy G. H., Littlewood J., Pölya G. Inequalities. N.Y.: Cambridge Univ. Press, 1934.

47. Hermes H., LaSalle J.P. Functional Analysis and Optimal Control. N.Y.-London: Academic Press, 1969.

48. Hitchcock F. L. The distribution of a product from several sources to numerous localities // J. of Mathematics and Physics. 1941. V. 20. P. 224-230.

49. Jackson D. Über die Genauigkett des Annäherung stetigen Funktionen durch ganze rationale Funktionen gegebenen Grades und trigonometrischen Summen gegebener Ordnung. Göttingen: Diss., 1911.

50. Karlin S. Oscillatory perfect splines and related extremal problems // Studies in Spline Functions and Approximation Theory / Eds. S. Karlin, C.A. Micchelli, A. Pinkus, I. J. Schoenberg. N.Y.: Academic Press, 1976. P. 371-460.

51. Landau E. Einige Ungleichingen für zweimal differentierbare Funktionen // Proc. London Math. Soc. 1913. V. 13. P. 43-49.

52. Levin V.L. General Monge-Kantorovich problem and its applications in measure theory and mathematical economics // Functional Analysis, Optimization and Mathematical Economics. N.Y.: Oxford Univ. Press, 1990. P. 141-176. 
53. Lorentz G. G. An inequality for rearrangements // Amer. Math. Monthly. 1953. V. 60. P. $176-179$.

54. Lorentz G. G. Bernstein Polynomials. Toronto: Univ. of Toronto Press, 1953.

55. Lorentz G. G., von Golitschek M., Makovoz Yu. Constructive Approximation. Advanced Problems. Berlin: Springer, 1996.

56. McCann R. Exact solutions to the transportation problem on the line. Preprint, 1998.

57. Monge G. Memoire sur la theorie des deblais et des remblais // Mem. Acad. Sci. Paris. 1781.

58. Muirhead R.F. Some methods applicable to identities and inequalities of symmetric algebraic functions of $n$ letters // Proc. Edinburgh Math. Soc. 1903. V. 21. P. 144-157.

59. Nikol'skii S. M. La série de Fourier d'une fonction dont be module de continuité est donné // Dokl. Akad. Nauk SSSR. 1946. V. 52. P. 191-194.

60. Papadimitriou C. H., Steiglitz K. Combinatorial Optimization: algorithms and complexity. N. J.: Prentice Hall, Englewood Cliffs, 1982.

61. Pinkus A. Some extremal properties of perfect splines and the pointwise Landau problem on the finite interval // J. of Approximation Theory. 1978. V. 23. № 2. P. 37-64.

62. Schrijver A. Theory of Linear and Integer Programming. V.2. N.Y.: John Wiley\& Sons, 1986.

Поступило в редакцию

27.XI. 1997 